\title{
Neurons burdened by DNA double strand breaks incite microglia activation through antiviral-like signaling in neurodegeneration.
}

Gwyneth Welch ${ }^{1,2}$, Carles Boix ${ }^{3}$, Eloi Schmauch ${ }^{3,4,5}$, Jose Davila-Velderrain ${ }^{3}$, Matheus B. Victor $^{1,2}$, Vishnu Dileep ${ }^{1,2}$, Lorenzo Bozzelli ${ }^{1,2}$, Qiao Su ${ }^{6}$, Jemmie Cheng ${ }^{1,2}$, Audrey Lee ${ }^{1,2}$, Noelle Leary ${ }^{1,2}$, Andreas Pfenning ${ }^{6}$, Manolis Kellis ${ }^{3,4}$, and Li-Huei Tsai ${ }^{1,2,4, *}$

1. Picower Institute for Learning and Memory, Massachusetts Institute of Technology, Cambridge, MA, USA.

2. Department of Brain and Cognitive Sciences, Massachusetts Institute of Technology, Cambridge, MA, USA.

3. Computer Science and Artificial Intelligence Laboratory, Massachusetts Institute of Technology, Cambridge, MA, USA.

4. Broad Institute of Harvard and MIT, Cambridge, MA, USA.

5. A. I. Virtanen Institute for Molecular Sciences, University of Eastern Finland, Kuopio, Finland

6. Carnegie Mellon University Departments of Computational Biology and Biology and Neuroscience Institute, Pittsburgh, PA, USA.

*To whom correspondence should be addressed: $\underline{1 \text { htsai@mit.edu }}$ 


\section{Summary}

3 It is unclear how age-associated DNA double strand break (DSB) accumulation in neurons

4 influences the progression of cellular senescence and neurodegenerative disease. Here, we

5 leverage mouse models of neurodegeneration, single-nucleus, bulk, and spatial transcriptomics

6 from Alzheimer's disease patients, mouse models, and primary neuron cultures to dissect the

7 immune signaling pathways initiated by DSB-bearing neurons that trigger neuroinflammation.

\section{Abstract}

9 DNA double strand breaks (DSBs) are linked to aging, neurodegeneration, and senescence ${ }^{1,2}$.

10 However, the role played by neurons burdened with DSBs in disease-associated

11 neuroinflammation is not well understood. Here, we isolate neurons harboring DSBs from the CK-

12 p25 mouse model of neurodegeneration through fluorescence-activated nuclei sorting (FANS),

13 and characterize their transcriptomes using single-nucleus, bulk, and spatial sequencing

14 techniques. We find that neurons harboring DSBs enter a late-stage DNA damage response marked

15 by the activation of senescent and antiviral-like immune pathways. We identify the NFkB

16 transcription factor as a master regulator of immune gene expression in DSB-bearing neurons, and

17 find that the expression of cytokines like $\mathrm{Cxcl10}$ and $\mathrm{Ccl} 2$ develop in DSB-bearing neurons before

18 glial cell types. Alzheimer's Disease pathology is significantly associated with immune activation

19 in excitatory neurons, and direct purification of DSB-bearing neurons from Alzheimer's Disease

20 brain tissue further validates immune gene upregulation. Spatial transcriptomics reveal that regions

21 of brain tissue dense with DSB-bearing neurons also harbor signatures of inflammatory microglia,

22 which is ameliorated by NFkB knock down in neurons. Inhibition of NFkB or depletion of Ccl2

23 and Cxcl10 in DSB-bearing neurons also reduces microglial activation in organotypic brain slice 
24 culture. In conclusion, we find that in the context of age-associated neurodegenerative disease,

25 DSBs activate immune pathways in neurons, which in turn adopt a senescence associated secretory

26 phenotype to elicit microglia activation. These findings highlight a novel role for neurons in the

27 mechanism of age-associated neuroinflammation.

\section{Introduction}

29 Loss of genomic integrity is linked to aging and neurodegeneration ${ }^{1,2}$. DNA damage repair

30 pathways are transcriptionally prominent in the aging brain, and many age-associated

31 neurodegenerative diseases exhibit both accumulation of DNA lesions and reduced DNA repair

32 efficiency ${ }^{3-7}$. The most toxic of these lesions, the DNA double strand break (DSB), can drive many

33 phenotypes of aging including senescence, mutation, and cell death. Postmitotic neurons are

34 particularly susceptible to these threats due to their long lifespan, high metabolic activity, and

35 limited DSB repair capacity. While DSB accumulation in neurons is a well-documented feature of

36 aging and neurodegeneration, the transcriptional profile adopted by such population of neurons

37 remains largely unknown.

38 The accumulation of DSBs is an early feature of Alzheimer's Disease (AD), suggesting that they

39 may act as an initiating lesion of toxicity ${ }^{8}$. Multiple mouse models of neurodegeneration

40 phenocopy increased DSBs at early pathological stages, including the P301S tauopathy model, the

41 inducible CK-p25 model, and the 3xTg and hAPP-J20 amyloid pathology models ${ }^{9-13}$. In addition,

42 induction of DSBs are sufficient to induce organismal aging and neurodegenerative phenotypes in

43 mice $^{14}$. Recent studies characterizing single and double strand breaks in postmitotic neurons reveal

44 that break location may underlie the toxicity of DNA damage in aging and neurodegenerative 
45 disease ${ }^{15,16}$. However, regardless of location, the downstream biological effects of DSB

46 accumulation in neurons are unclear.

47 Here, we aimed to characterize the biological consequences of DSB accumulation in neurons. We

48 also investigated how this impacts mechanisms of neuroinflammation in age-associated

49 neurodegenerative disease. We utilized fluorescence-activated nuclei sorting (FANS) followed by

50 bulk and single-nucleus RNA-sequencing to transcriptionally characterize neurons burdened with

51 DSBs in the CK-p25 mouse model of neurodegeneration. We found that DSB-bearing neurons

52 activate innate immune signaling pathways reminiscent of those expressed by senescent cells and

53 virally infected neurons ${ }^{17-24}$, and that this is accompanied by degradation of neuronal identity. The

54 gene expression patterns of DSB-burdened neurons were enriched in excitatory neurons from AD

55 postmortem human brain. Purified DSB-bearing neurons from the human brain also exhibited an

56 enrichment of immune gene signatures. Spatial transcriptomics of the CK-p25 forebrain revealed

57 signatures of microglial inflammation were closely associated with DSB-bearing neurons.

58 Correspondingly, suppression of the NFkB transcription factor in neurons impacted measures of

59 neuroinflammation in microglia at early stages of disease. Together, these data establish a novel

60 signaling relationship between neurons burdened with DSBs and microglia in age-associated

61 neurodegenerative disease.

62 Results

63 Identification of DSB-bearing neurons at early stages of disease in a mouse model of 64 neurodegeneration.

65 We utilized the CK-p25 mouse model of inducible neurodegeneration to understand how DSB-

66 bearing neurons contribute to disease development. In these mice, the CamkII promoter drives the 
67 expression of the neurotoxic protein fragment p25 through a doxycycline-off system ${ }^{25}$. P25 is the 68 calpain-cleaved product of p35, an activator of cyclin dependent kinase 5 (Cdk5). Previously, we

69 determined that the first pathologies observed in these mice are increased DSBs in neurons ${ }^{9}$ and 70 activation of microglia ${ }^{26}$. These pathologies occur 1-2 weeks after the onset of p25 expression

71 when mice are taken off doxycycline (dox). Intracellular amyloid-beta accumulation occurs as

72 early as 2-3 weeks after induction ${ }^{27}$. Neuronal loss, learning deficits, and tau hyperphosphorylation

73 are also observed in the following 4-12 weeks ${ }^{25,27,28}$. These pathological events are inducible and

74 occur in a highly concerted and predictable manner ${ }^{29}$.

75 The phosphorylation of the histone variant H2A.X ( $\gamma \mathrm{H} 2 \mathrm{AX})$ by ATM kinase occurs rapidly after

76 DSB detection. This post-translational histone modification is essential for efficient DSB

77 recognition and repair, and is a robust DSB biomarker ${ }^{30}$. Using flow cytometry, we identified a

78 distinct population of $\gamma \mathrm{H} 2 \mathrm{AX}$-positive nuclei at the two-week time point in the CK-p25 cortex,

79 but not in the CK control cortex (Figure 1a). CK mice express the CamkII-driven tetracycline

80 transactivator (tTA), but not p25. A timeline analysis was also performed to determine when

$81 \gamma \mathrm{H} 2 \mathrm{AX}$-positive nuclei begin to accumulate in the CK-p25 cortex. $\gamma \mathrm{H} 2 \mathrm{AX}$-positive nuclei were

82 detectable as early as one week after induction (1.038 $\pm 0.2627 \%$ population), peaked at two

83 weeks $(4.614 \pm 0.9416 \%$ population), and gradually decreased thereafter (Figure $1 \mathrm{~b}$ ). The

84 significant reduction in $\gamma \mathrm{H} 2 \mathrm{AX}$-positive nuclei at six weeks corresponds with previous

85 observations of neuronal loss in this model, suggesting that $\gamma \mathrm{H} 2 \mathrm{AX}$-positive cells degenerate by

86 six weeks ${ }^{25,28}$. We were able to observe similar $\gamma \mathrm{H} 2 \mathrm{AX}$-positive population dynamics using

87 immunofluorescent microscopy (Supplementary Figure 1a,b). We did not detect $\gamma \mathrm{H} 2 \mathrm{AX}$-positive

88 nuclei after only four days off dox, suggesting that one week is the earliest time point at which this

89 population appears. To validate DNA damage response pathways were active in these nuclei, we 
confirmed co-immunoreactivity for phosphorylated ATM kinase (pATM) (Supplementary Figure

$911 \mathrm{c}, \mathrm{d})$.

92 We found that all $\gamma \mathrm{H} 2 \mathrm{AX}$-positive nuclei stained positively for CamkIIa, a marker of excitatory

93 neurons (Supplementary Figure 1e,f). Interestingly, at the two week time point, only $57.37 \pm 8.822$

$94 \%$ of $\gamma \mathrm{H} 2 \mathrm{AX}$-positive nuclei also stained positive for $\mathrm{NeuN}$, a general marker for forebrain neurons

95 (Supplementary Figure 1e,g). Degradation of neuron cell type identity is observed within aging

96 and Alzheimer's disease patients ${ }^{31,32}$, and loss of NeuN expression has been proposed to be an

97 indicator of declining neuronal health ${ }^{33}$. Indeed, reduced $\mathrm{NeuN}$ expression or reduced $\mathrm{NeuN}$

98 immunoreactivity is an established feature of neuronal damage ${ }^{34-37}$. Furthermore, $\gamma \mathrm{H} 2 \mathrm{AX}$-positive

99 nuclei did not overlap with markers of microglia (Iba1), astrocytes (GFAP), or oligodendrocytes

100 and oligodendrocyte precursor cells (Olig2), indicating that they were not of glial origin

101 (Supplementary Figure 1h,i). Thus, we classified nuclei based on their NeuN and $\gamma \mathrm{H} 2 \mathrm{AX}$

102 immunoreactivity. This resulted in four distinct populations: $\gamma \mathrm{H} 2 \mathrm{AX}$-negative NeuN-positive

103 ("Baseline” neurons), $\gamma \mathrm{H} 2 \mathrm{AX}$-positive NeuN-positive (Stage 1 neurons), $\gamma \mathrm{H} 2 \mathrm{AX}$-positive NeuN-

104 negative (Stage 2 neurons), and $\gamma \mathrm{H} 2 \mathrm{AX}$-negative NeuN-negative (putative non-neuronal cells,

105 which we refer to as "Other") (Supplementary Figure 1g). We used fluorescence activated nuclei

106 sorting (FANS) followed by bulk RNA sequencing to transcriptionally profile each population

107 (Figure 1c). All nuclei were collected for sequencing at the two-week time point, which was when

108 we observed the peak density of $\gamma \mathrm{H} 2 \mathrm{AX}$-positive nuclei.

109 Both Stage 1 and Stage 2 populations expressed more p25 transgene compared to other populations

110 (Supplementary Figure 1j). CK-p25 mice exclusively express p25 in excitatory forebrain neurons,

111 as it relies on the expression of tTA driven by the CamkII promoter. Because the p25 transgene is

112 fused to GFP, we performed staining to validate this finding. We found that $85.74 \pm 0.055 \%$ of 
$113 \gamma \mathrm{H} 2 \mathrm{AX}$-positive cells, regardless of NeuN immunoreactivity, were also GFP-positive

114 (Supplementary Figure 1k,1). To verify Stage 2 neuronal identity independent of transgene

115 expression, we also stained for Neurod1, a neuronal transcription factor. We found that $81.96 \pm$

$1160.046 \%$ of $\gamma \mathrm{H} 2 \mathrm{AX}$-positive cells stained positively for Neurod1, regardless of $\mathrm{NeuN}$

117 immunoreactivity (Supplementary Figure 1m,n). Together, our neuron marker and transgene

118 expression analysis indicated that both Stage 1 and Stage 2 populations were likely excitatory

119 neurons engaged in a DSB response.

120 Differential gene expression in DSB-bearing neurons.

121 We performed two differential expression analyses to characterize gene expression changes in

122 these populations: Stage 1 vs. CK-p25 Baseline, and Stage 2 vs. CK-p25 Baseline. Large

123 transcriptional changes were observed in both contrasts. 3,031 upregulated and 717 downregulated

124 transcripts were identified in Stage 1, and 5,055 upregulated and 3,792 downregulated transcripts

125 were identified in Stage $2\left(\log _{2}\right.$ fold change $\geq 1$, adjusted p-value $\left.<0.05\right)$ (Supplementary Figure

126 2a-c). Gene set enrichment analysis (GSEA) $)^{38}$ revealed that both Stage 1 and Stage 2 neurons

127 displayed a significant enrichment for genes implicated in DSB repair, apoptotic signaling, and

128 cell cycle, and a significant reduction in synaptic function processes (Figure 1d). These pathways

129 were previously found deregulated in both the CK-p25 mouse and AD human brain

130 tissue $9,26,29,39,40$.

131 Remarkably, we also found that a number of innate immune pathways were enriched in Stage 1

132 and Stage 2 neurons. This included gene ontology terms 'Senescence-Associated Secretory

133 Phenotype (SASP)', 'cytosolic sensors of pathogen-associated DNA', and 'positive regulation of

134 innate immune response' (Figure 1d). Upon closer inspection, we observed an enrichment of genes 
135 linked to DSB-mediated activation of SASP signaling, particularly in Stage 2 neurons. This

136 included nucleic acid sensors such as Cgas and Zbp1, NFkB subcomponents Rela and Relb, SASP

137 factors Il6, Il15, Ccl2, Cxcl10, and interferon stimulated genes Isg15 and Ifitm3 (Figure 1e).

138 Notably, a number of these genes are expressed in neurons following viral infection ${ }^{23,24}$. These

139 genes are also classic hallmarks of senescence, suggesting that DSB accumulation elicits senescent

140 and anti-viral-like responses in neurons.

141 Because the CK-p25 model is characterized by the development of type-I interferon-reactive 142 microglia specifically by two weeks induction ${ }^{26}$, we wanted to determine if DSB-bearing neurons 143 express cytokines before microglia. To do this, we performed RNAscope multiplexed fluorescent 144 in-situ hybridization in the CK-p25 cortex after one and two weeks of induction. We focused our 145 profiling on $\mathrm{Cxcl10}$ and $\mathrm{Ccl} 2$ because these pro-inflammatory chemotactic molecules were highly 146 expressed in Stage 2 neurons and are known to be secreted by neurons upon viral infection ${ }^{23,41-44}$.

147 We also performed RNAscope for the excitatory neuron marker Camk2a to again confirm if $148 \gamma \mathrm{H} 2 \mathrm{AX}$-positive nuclei were of neuronal origin. In agreeance with our RNAseq and flow 149 cytometry data, $98.81 \pm 1.19 \%$ of $\gamma \mathrm{H} 2 \mathrm{AX}$-positive nuclei were also Camk2a-positive 150 (Supplementary Figure 2d). This high positivity rate reveals that $\gamma \mathrm{H} 2 \mathrm{AX}$-positive nuclei are nearly 151 exclusively neurons. RNAscope analysis also revealed that DSB-bearing neurons were the only 152 cells to express $\mathrm{Cxcl10}$ and $\mathrm{Ccl} 2$ at the one-week time point. In contrast, $\mathrm{Cxcl10}$ and $\mathrm{Ccl} 2$ gene 153 expression was highly enriched in other cell types at the two-week time point (Figure 1f, 154 Supplementary Figure 2e). This indicated that expression of these chemokines in $\gamma \mathrm{H} 2 \mathrm{AX}$-positive 155 neurons precedes their expression in glial cells, and that cytokine secretion from DSB-bearing 156 neurons may be an early mechanism of glial cell recruitment and activation in the CK-p25 brain. 
157 To identify the master regulators of DSB-associated neuronal immune signaling, we performed

158 transcription factor enrichment analysis using Enrichr ${ }^{45-47}$. A subset of immune pathway genes

159 was extracted from the significantly upregulated genes in Stage 2 neurons for analysis. The overlap

160 between this immune gene module and transcription factor target genes was then calculated.

161 Multiple subunits of the NFkB complex were consistently enriched across Enrichr transcription

162 factor libraries, including Rela, Nfkb1, and Relb (Supplementary Table 1). Notably, the NFkB

163 transcription factor plays a well-established role in SASP activation and the DSB response ${ }^{19,20,22}$.

164 We chose to focus on Rela, also known as p65, because it is a core member of the canonical NFkB

165 complex, and it was most frequently enriched in the Enrichr analysis. We stained for p65 in two-

166 week CK and CK-p25 cortices. NFkB is normally sequestered in the cytosol, but translocates to

167 the nucleus to form an active complex upon cellular insult. Nuclear p65 intensity was significantly

168 higher in $\gamma \mathrm{H} 2 \mathrm{AX}$-postive neurons compared to other $\gamma \mathrm{H} 2 \mathrm{AX}$-negative cells (Figure 1g),

169 supporting evidence of increased NFkB transcriptional activity.

\section{Single cell RNA-sequencing if DSB-bearing neurons.}

171 Notably, inflammatory gene expression was lower in Stage 1 neurons compared to Stage 2 neurons

172 (Figure 1e), and more Stage 1 neurons were identified at the one-week time point compared to

173 Stage 2 neurons (Supplementary Figure 2f,g). This suggested that the Stage 2 population may

174 develop after Stage 1, which aligned with our initial prediction when defining these distinct cellular

175 states based on NeuN-reactivity. To better understand the relationship between Stage 1 and Stage

1762 neurons, we performed single nucleus RNA-sequencing on each FANS-gated population at both

177 one and two-week timepoints (Figure 1c). A total of 1,357 single nucleus libraries were prepared

178 using SMARTseq2 chemistry. Following quality control measures (see methods), 889 libraries

179 remained for downstream analysis (Figure 1h, Supplementary Figure 3a). Cells were classified 
into major cell type clusters based on marker gene expression, resulting in the identification of 521 cells (OPCs), and 50 microglia. We did not detect any astrocytes.

Remarkably, the majority of Stage 2-gated nuclei formed their own cell type cluster (Figure 1h).

184 While we did identify 13 Stage 2-gated nuclei in microglia and oligodendrocyte clusters, all of the nuclei in question came from one CK-p25 mouse (Supplementary Figure 3b,c). None of the other

186 five CK-p25 mice had Stage 2-gated nuclei fall into glial clusters. Combined with our previous

187 cell type immunostaining analysis (Supplementary Figure 1h,i), we concluded that the immune 188 signature identified in the Stage 2 population was not likely to be driven by contamination from microglia or oligodendrocytes. Compared to other neuronal clusters, the in silico Stage 2 cluster was significantly enriched for the FANS-gated Stage 1 and Stage 2 gene signatures

191 (Supplementary Figure 3d). Interestingly, the Stage 2 cluster expressed only moderate levels of the excitatory neuron cluster markers Camk2a, Grial, and Syt2, and lacked marker genes of other

193 canonical cell types such as inhibitory neurons (Gad1, Gad2), astrocytes (GFAP), microglia 194 (Cd33, Csflr), oligodendrocytes (Plp1, Mbp), and OPCs (Bcan). Instead, the Stage 2 cluster 195 expressed marker genes indicative of senescence, including Cdknla, and Ubb (Figure 1i). These 196 distinctive cell type markers further suggested to us that the Stage 2 cluster represented a 197 population of DSB-bearing neurons engaged in a senescence-like inflammatory response.

198 To further examine Stage 1 and Stage 2 cell type heterogeneity, we subclustered all neuronal cells.

199 This resulted in the identification of four excitatory neuron subclusters (Ex0-3), and two inhibitory 200 neuron subclusters (In0, In1) (Figure 1h). Stage 1 cells were enriched in subclusters Ex2 and Ex3 201 compared to Ex0 and Ex1 (Supplementary Figure 3c). Subclusters Ex2 and Ex3 were also marked 
203 Ex3 cells were engaged in a DSB repair response (Figure 1i).

204 Interestingly, these DNA repair-associated genes were not expressed in the Stage 2 cluster.

205 Combined with the enrichment for the classical senescence marker Cdkn1a, this suggested that

206 Stage 2 cells may be engaged in a later-stage response to DSBs. This motivated us to perform

207 trajectory analysis. The single cell analysis package Monocle $3^{48-50}$ was used to order cells from

208 subclusters Ex0,1,2,3 and Stage 2 along a pseudotime, which was generated through a learned

209 trajectory of subcluster gene expression differences (Figure 1j). Both CK and CK-p25 cells were

210 used for this analysis. Next, we identified genes that changed as a function of pseudotime. We

211 observed sequential enrichment of Stage 1 and Stage 2 gene signatures along pseudotime (Figure

212 1k). The expression of Stage 2 signature genes specifically involved in immune response

213 (Immune) were also enriched at the end of pseudotime (Figure 1k). We found that genes associated

214 with neuronal identity such as Gria2 and Kalrn were most highly expressed at the beginning of

215 the trajectory (Figure 1j). Meanwhile, genes associated with DNA repair like Hjurp, Ligl, and

$216 N e k 3$ peaked along the middle, and Cdknla expression peaked at the end of the trajectory. We also

217 observed late-stage expression of Apoe, supporting previous observations that Apoe expression

218 increases in neurons following injury ${ }^{51}$.

\section{DSBs elicit innate immune signaling in neurons.}

220 To test if this late-stage DSB response signature is triggered by DSBs or represents a unique

221 response mounted by CK-p25 mice, we treated murine primary neuron cultures with $50 \mathrm{uM}$

222 etoposide (ETP). ETP produces DSBs by inhibiting topoisomerase-II function, thus preventing the

223 re-ligation of cleaved DNA-TopII complexes (Figure 2a). This dose of ETP was consistent with 
224 previous studies that used ETP to investigate DSB-elicted immune activity in vitro ${ }^{52}$. We also 225 performed cell type immunostaining analysis to assess the cell type composition of our primary 226 cultures. $82.87 \%$ of cells were identified as mature neurons (NeuN-positive), and another $9.31 \%$ 227 were neural precursor cells (Nestin-positive), (Supplementary Figure 4a). A trace number of cells 228 were also identified as astrocytes (GFAP-positive, 2.46\%) and oligodendroglia (Olig2-positive 229 Nestin-negative, 1.35\%).

230 RNA sequencing was performed to profile the transcriptional changes occurring in primary 231 neurons after ETP treatment (Figure 2b). There was a significant upregulation of 7,532 transcripts 232 and downregulation of 7,106 transcripts compared to vehicle-treated control neurons (DMSO) 233 (adjusted p-value $<0.05$ ) (Supplementary Figure 4b-d). Notably, GSEA revealed that biological 234 pathways mediating senescent and antiviral activity such as 'interferon alpha response,' and 'il6 235 jak stat3 signaling,' were enriched in ETP-treated cultures (Figure 2c). Furthermore, many of the 236 cytokines upregulated in Stage 2 neurons were also upregulated in ETP-treated neurons, including 237 Ccl2, Cxcl10, Cxcl16, and Il6 (Supplementary Table 2). Stage 1 and Stage 2 gene signatures were 238 both significantly enriched $(\mathrm{p}=0.00064 \& \mathrm{p}=0.0081$ respectively) (Figure $2 \mathrm{~d}$, Supplementary 239 Figure 4e). To perform a more detailed comparison of Stage 1 \& 2 signature genes to ETP DEGs, 240 we generated a Venn-Diagram (Figure 2e). 11.87\% of all DEGs (total DEGs from all datasets)

241 were shared between ETP, Stage 1 and Stage 2 conditions. This overlap was enriched for DNA 242 damage repair genes such as Parp10, Tdp2, Fanca, and Rad54l. A further 12.87\% of all DEGs 243 were shared between ETP and Stage 2 conditions. This overlap was enriched for immune genes 244 such as Rela, Nfkbia, and Irfl.

245 To confirm immune genes were expressed by neurons and not by other trace cell types in our 246 primary cultures, we performed RNAscope for Ccl2 and the gene encoding NeuN, Rbfox3. Cells 
247 that were Rbfox3-positive had significantly more Ccl2 puncta than Rbfox3-negative cells

248 (Supplementary Figure 4f). p65 nuclear intensity was also significantly increased in ETP-treated

249 neurons (Figure 2a). A NeuN co-stain was used to confirm p65 activation was derived from

250 neurons.

251 We also used an independent method of DSB induction in neurons by treating primary cultures

252 with 10 Gy of X-ray irradiation (Figure 2f). After a 24-hour recovery period, we performed RT-

253 qPCR to assess the mRNA levels of the Stage 2 marker gene Cdknla, and the antiviral signaling

254 genes $\mathrm{Ccl} 2$ and $\mathrm{Cxcl10.10}$ Gy of X-ray irradiation significantly increased the expression of all

255 three of these genes (Figure $2 \mathrm{~g}$ ).

\section{A DSB-associated immune signature is conserved in human neurons.}

257 We next sought to determine if signatures of DSB-bearing neurons can also be detected in the

258 human brain. First, we used the Stage 2 gene signature to identify neurons with DSB-associated

259 immune activation in a previously published snRNA-seq dataset of individuals with and without

260 AD pathology (Figure 3a) ${ }^{53}$. We assessed Stage 2 gene expression in all major brain cell types:

261 excitatory neurons, inhibitory neurons, astrocytes, oligodendrocytes, oligodendrocyte precursor

262 cells, and microglia. For each cell type, genes were ranked based on their expression and

263 correlation to 'global pathology,' a variable quantifying three major AD pathologies: neuritic

264 plaques, diffuse plaques, and neurofibrillary tangles ${ }^{54}$. Genes positively correlated with global

265 pathology for each cell type were then sampled for the enrichment of Stage 2 genes (Figure 4b).

266 The strongest enrichment of Stage 2 genes was identified in excitatory neurons $(\mathrm{p}=8.7 \mathrm{E}-19)$, and

267 inhibitory neurons $(\mathrm{p}=7.7 \mathrm{E}-7)$. Astrocytes and oligodendrocytes were also found to have a 268 significant enrichment of Stage 2 genes correlating with global pathology, but to a much lesser 
extent (Figure 4b), (Bonferroni adjusted p-value $<0.01$ ). Therefore, while we cannot exclude the

270 possibility that human astrocytes and oligodendrocytes may carry some disease-associated

271 enrichment for Stage 2 genes, the Stage 2 signature is largely driven by neurons in this dataset.

272 When assessing the Stage 2 genes positively correlated with global pathology in excitatory

273 neurons, we again observed an enrichment of immune-related biological pathways, including the

274 genes Ccl2-like receptor (CCRL2), CD74, CXCL16, and $A P O E$ (Figure 4c,d).

275 To validate the enrichment of DSB-bearing neurons identified in our snRNA-seq analysis, we 276 sorted $\gamma \mathrm{H} 2 \mathrm{AX}$-positive and $\gamma \mathrm{H} 2 \mathrm{AX}$-negative NeuN-positive and NeuN-negative nuclei from the

277 postmortem temporal cortex of 6 individuals, and then performed bulk RNA sequencing (Figure 278 4e, Supplementary Figure 5a-c). These individuals were part of the Massachusetts Alzheimer's

279 Disease Research Center (MADRC) cohort, and were divided by Braak score as either AD (Braak 280 4-6), or non-AD (Braak 1-3). Age, sex, and other biological variables for each individual are 281 available in Supplementary Table 3. To assess the cell type specificity of our sorted populations, 282 we examined the expression of several cell type marker genes (Supplementary Figure 5d-f). Unlike 283 the Stage 2 nuclei from the CK-p25 mice, $\gamma \mathrm{H} 2 \mathrm{AX}$-positive NeuN-negative nuclei from the human 284 brain were enriched for glial markers (Supplementary Figure 5e,f). This indicated to us that human 285 glial cells also accumulate DSBs, and that bulk $\gamma \mathrm{H} 2 \mathrm{AX}$-positive NeuN-negative nuclei were likely 286 to contain a heterogenous population of many different cell types. Thus, to ensure we were 287 analyzing neuronal gene expression, we focused our analysis on $\gamma \mathrm{H} 2 \mathrm{AX}$-positive NeuN-positive 288 nuclei.

289 We assessed Stage 1 and Stage 2 gene signature activity in NeuN-positive nuclei from AD and 290 non-AD human brains (Figure 4f). $\gamma \mathrm{H} 2 \mathrm{AX}$-positive neurons displayed modest enrichment for the 291 CK-p25 Stage 1 and Stage 2 genes ( $p=0.31$ and $p=0.04$ respectively) (Figure 4g). Comparing 
samples by $\mathrm{AD}$ status and $\gamma \mathrm{H} 2 \mathrm{AX}$ status further revealed that Stage 1 and Stage 2 gene activity was markedly enriched in $\gamma \mathrm{H} 2 \mathrm{AX}$-positive neurons from AD individuals $(\mathrm{p}=0.0075$ and $\mathrm{p}=0.0021$, respectively) (Figure 4h,i). Furthermore, when contrasting all $\gamma \mathrm{H} 2 \mathrm{AX}$-positive neurons against all $\gamma \mathrm{H} 2 \mathrm{AX}$-negative neurons, we found that the average $\log _{2}$ fold change values for both Stage 1 and Stage 2 gene sets were positive, and significantly higher than the average $\log _{2}$ fold change values

297 for all other genes (Supplementary Figure 5g). We also assessed the transcriptional similarity 298 between human $\gamma \mathrm{H} 2 \mathrm{AX}$-positive neurons and ETP-treated primary neurons. Again, we found 299 striking similarities between the DSB signature produced in the human brain, and the DSB 300 signature generated by ETP treatment of primary neurons (Supplementary Figure 5h).

301 To further verify the enrichment of immune activation in DSB-bearing neurons from brains with $\mathrm{AD}$ pathology, we used another cohort of $\mathrm{AD}$ brain tissue to assess nuclear p65 expression in NeuN-positive neurons with high versus low burdens of DSBs. We acquired 4 prefrontal cortex tissue samples from individuals belonging to the Religious Orders Study cohort. All individuals analyzed exhibited AD pathology, described in Supplementary Table 4. To bin neurons by DSB burden, we analyzed $\gamma \mathrm{H} 2 \mathrm{AX}$ expression in NeuN-positive nuclei. The median $\gamma \mathrm{H} 2 \mathrm{AX}$ expression was calculated for each brain, and used to split neurons into the high or low DSB condition

308 (Supplementary Figure 6a). Neurons with high DSB burden consistently expressed higher levels of nuclear p65 compared to neurons with low DSB burden (Figure 3j,k, Supplementary Figure 6b). In summary, through the analysis of an independent snRNA-seq dataset, FANS RNA-sequencing,

311 and immunohistochemistry, we found that SASP and antiviral-like pathways were active in human

312 DSB-bearing neurons. Furthermore, this neuronal immune signature was further amplified in the 313 context of AD pathology, suggesting that it may serve a functional role in disease-associated 314 neuroinflammation. 


\section{DSB-bearing neurons stimulate glial activation.}

316 We have previously published an in-depth characterization of reactive microglia in CK-p25 mice 317 using snRNA-seq ${ }^{26}$. We wondered if these microglia were responding to immune signaling from

318 DSB-bearing neurons. To address this, we performed $\gamma \mathrm{H} 2 \mathrm{AX}$ immunostaining followed by spatial

319 transcriptomics on two-week induced CK-p25 brains (Figure 5a). The capture areas of 10X Visium

320 spatial transcriptomics slides are large enough to encompass the transcriptional profile of 3-5 cells

321 in mouse brain tissue. Therefore, we hypothesized that capture areas with $\gamma \mathrm{H} 2 \mathrm{AX}$ signal would

322 also contain transcripts from nearby microglia. First, we identified 23 spatial clusters across three

323 CK and four CK-p25 coronal sections (Figure 4b,c, Supplementary Figure 7a,b). Next, we used a

324 binary classification system to identify capture areas with $\gamma \mathrm{H} 2 \mathrm{AX}$ signal past a given threshold

325 (See Methods, Figure 4d,e, Supplementary Figure 7c). To determine if the reactive microglia

326 characterized in Mathys et al., 2017 were enriched in $\gamma \mathrm{H} 2 \mathrm{AX}$-positive capture areas, we performed

327 a differential comparison between all $\gamma \mathrm{H} 2 \mathrm{AX}$-positive capture areas and all $\gamma \mathrm{H} 2 \mathrm{AX}$-negative

328 capture areas. Using GSEA, we identified a robust enrichment of the microglia signature $(1,383$

329 significantly upregulated genes in reactive microglia compared to homeostatic microglia) within

$330 \gamma \mathrm{H} 2 \mathrm{AX}$-positive capture areas compared to $\gamma \mathrm{H} 2 \mathrm{AX}$-negative capture areas (Supplementary Figure

331 8a). This indicated that overall, the reactive microglia signature characterized in Mathys et al.,

3322017 was significantly associated with $\gamma \mathrm{H} 2 \mathrm{AX}$-positive neurons.

333 By mapping the density of $\gamma \mathrm{H} 2 \mathrm{AX}$-positive capture areas across all spatial clusters, we further

334 prioritized distinct spatial clusters enriched for $\gamma \mathrm{H} 2 \mathrm{AX}$-positive capture areas (Figure 4d,

335 Supplementary Figure $8 \mathrm{~b}$ ). We focused on spatial clusters with $\gamma \mathrm{H} 2 \mathrm{AX}$-positive capture areas

336 comprising $20 \%$ or more of total capture areas (Supplementary Figure $8 \mathrm{~b}$ ). This included clusters

33716 and 18, which corresponded to CA1,2,3 and the dentate gyrus respectively, and clusters 2,5,6,7, 
which were primarily localized to the cortex. Differential comparisons of $\gamma \mathrm{H} 2 \mathrm{AX}$-positive capture areas and $\gamma \mathrm{H} 2 \mathrm{AX}$-negative capture areas within each cluster of interest revealed a significant enrichment of the reactive microglia signature (Supplementary Figure 8a). Notably, many of the marker genes for reactive microglia displayed expression patterns that visually correlated with $\gamma \mathrm{H} 2 \mathrm{AX}$-positive capture areas, including the galectin gene $L g a l s 3 b p$, lipase $L p l$, cystatin $C s t 7$, and MHC Class I gene H2-D1 (Figure 4f). Genes also enriched in DSB-bearing neurons, such as

344 Cxcl10 and Ifitm3 was also closely correlated with $\gamma \mathrm{H} 2 \mathrm{AX}$-positive capture areas (Supplementary

345 Figure 9). Collectively, this spatial transcriptomic analysis indicated that reactive microglia are

346 closely associated with $\gamma \mathrm{H} 2 \mathrm{AX}$-positive neurons, suggesting the occurrence of neuro-immune

347 communication between damaged neurons and microglia.

To determine if microglia responded to DSB-mediated immune signaling, we sought to inhibit a master regulator of immune gene expression in neurons. Our data revealed that NFkB transcription factor activity is increased in DSB-bearing neurons, as are the genes downstream of NFkB

351 activation. Therefore, we focused on inhibiting NFkB transcriptional activity. To achieve this in 352 the CK-p25 mouse, we opted for brain-wide viral delivery of shRNAs targeting p65 ${ }^{55}$. We 353 performed retro-orbital injections of PHP.eb AAV shp65-RFP or Scramble-RFP into CK-p25 354 mice. CK control mice were injected with phosphate-buffered saline. Two weeks after injections, 355 mice were taken off doxycycline and induced for two weeks (Figure 4g).

357 were the primary targets of shRNA expression, and confirming the previously reported tropism of 358 PHP.eb for neuronal infectivity (Supplementary Figure 10a,b) ${ }^{55}$. The reduced expression of p65 in 359 mice was confirmed via qPCR and immunofluorescent staining (Supplementary Figure 10c-e). 360 The number of $\gamma \mathrm{H} 2 \mathrm{AX}$-positive neurons remained the same between p65 knock-down (p65kd) 
and Scramble mice (Supplementary Figure 10f,g). Next, we sorted $\gamma \mathrm{H} 2 \mathrm{AX}+$ and $\gamma \mathrm{H} 2 \mathrm{AX}-\mathrm{RFP}+$ neurons from both p65kd and Scramble mice to assess changes in immune gene expression

363 (Supplementary Figure 11a). RT-qPCR analysis revealed that a number of immune genes were

364 downregulated in DSB-bearing neurons from p65kd mice compared to Scramble, including Ccl2, confirmed via RNAscope (Supplementary Figure 11b,c). This indicated p65kd was able to alter neuron immune gene signatures.

To determine if suppressing neuron immune genes rescued microglial activation, we performed immunofluorescent staining with the microglia marker Iba1. We found that $\mathrm{p} 65 \mathrm{kd}$ mice showed a reduced number of microglia (Figure 4i), which was confirmed through flow cytometry analysis

371 of PU.1-positive nuclei from the entire cortex (Supplementary Figure 11d,e). We also found that

372 microglia soma area was significantly reduced, but differences in branch length and end points

373 were not statistically significant (Figure 4i). These data show that suppressing neuron immune

374 signaling significantly affects the number and morphology of microglia.

375 To determine if changes in microglial number and morphology were accompanied with changes

376 in gene expression, we performed RNA sequencing on sorted PU.1-positive nuclei from CK

377 control, CK-p25-scramble, and CK-p25-p65kd cortex (Figure 4g, Supplementary Figure 11d).

378 Differential analysis comparing microglia from p65kd and Scramble cortices revealed the 379 upregulation of 627 transcripts and downregulation of 160 transcripts (Figure 4j, Supplementary

380 Figure 11i,j). Interestingly, pathways related to synaptic activity and membrane organization were 381 significantly upregulated in microglia from p65kd mice. This included calcium channels Cacnalg,

382 Cacnalc, and Cacnb2, and sodium channel Scn1a, suggesting p65kd microglia may return to a 383 more homeostatic state embodied by CK microglia (Figure $4 \mathrm{j}$ ). In contrast, the genes that were 
384 downregulated in microglia from p65kd mice were involved in cell killing and antigen processing,

385 such as $H 2-Q 2, \operatorname{Lag} 3, \mathrm{Ccr} 5$, and $C c r 2$. Notably, the Ccr2 gene encodes the receptor for $\mathrm{Ccl} 2$,

386 further implying an alteration of the Cc12-Ccr2 axis (Figure 4k,1). We also observed a

387 downregulation of genes related to lipoprotein processing (Figure $4 \mathrm{k}, 1$ ). Together, these results

388 suggest that neuron immune signaling disrupts microglia homeostatic activity, and activate cell

389 killing and antigen processing mechanisms in microglia.

390 Finally, to determine if specific cytokines expressed and secreted by DSB-bearing neurons play a 391 role in microglia activation, we transitioned to our in vitro ETP model of DNA damage. First we

392 wanted to understand how conditioned media from ETP-treated neurons affect microglia 393 morphology. To do this, we generated organotypic acute brain slice cultures from Cx3cr1-GFP 394 mice, which express GFP in all microglia. After ETP treatment and washout with PBS, neurons 395 recovered in fresh media for 24 hours. This conditioned media was then collected and applied to 396 Cx3cr1-GFP brain slices for 6 hours (Figure 5a). The conditioned media from ETP-treated neurons 397 reduced microglia branch length (Figure 5b,c), but did not significantly affect the number of 398 endpoints per microglia (Figure 5d). We also observed increased soma area (Figure 5e). Treating 399 primary neurons with an NFkB activation inhibitor before and during ETP treatment significantly 400 reduced the soma area of Cx3crl-GFP-positive microglia, and increased branch length and end401 points (Figure 5b-e). Conditioned media from ETP-treated neurons did not elicit a robust 402 difference in the number of microglia analyzed per image (Figure 5f). These data further 403 demonstrate NFkB activity as a significant mediator of immune signaling in DSB-bearing neurons.

404 Notably, conditioned media from ETP-treated neurons was significantly enriched for Cxcl10 and $405 \mathrm{Ccl} 2$ (Figure 5g,h). Furthermore, the NFkB activation inhibitor reduced the concentration of these 406 cytokines (Figure 5g,h). Previously, we found that DSB-bearing neurons are the first cells to 

express Cxcl10 and Ccl2 in the CK-p25 cortex (Figure 1f, Supplementary Figure 2e). These cytokines induce the migration of microglia and macrophages to sites of viral infection, traumatic

409 brain injury, and gliomas, suggesting that they may be primary constituents of immune signaling

410 in DSB-bearing neurons ${ }^{23,43,44,56,57}$. To determine if they played a role in DSB-mediated microglia

411 activation, we immunodepleted $\mathrm{Ccl} 2$ or $\mathrm{Cxcl10}$ from conditioned media (Figure 5i). Both Cc12

412 and Cxcl10 depletion increased branch length and end-points per microglia compared to IgG

413 control depletion (Figure 5j,k,1). Interestingly, $\mathrm{Ccl} 2$ depletion reduced microglia soma area, but

414 Cxcl10 depletion did not (Figure 5m). Neither immunodepletion had an effect on microglia

415 number (Figure 5n). Combined, this suggests that while both $\mathrm{Ccl} 2$ and $\mathrm{Cxcl} 10$ elicit morphological

416 changes in microglia, they may play differential roles in microglia activation. Notably, both Cc12

417 and Cxcl10 are upregulated in aging and AD pathogenesis, suggesting their signaling activity via

418 DSB-bearing neurons could have significant effects on neuroinflammation ${ }^{44,58,59}$. Together, our

419 data indicate $\mathrm{Ccl} 2$ and $\mathrm{Cxcl} 10$ play important roles in recruiting and activating microglia to

420 neurons burdened with DSBs. This establishes a novel role for neuronal communication with

421 microglia in the context of age-associated neurodegenerative disease, and uncovers a new facet of

422 DSB toxicity in mature postmitotic neurons.

\section{Discussion}

424 Substantial evidence supports a causal role for microglia and neuroinflammation in AD

425 pathogenesis ${ }^{29,60,61}$. For example, many $\mathrm{AD}$ risk genes and genomic loci are most active in

426 microglia ${ }^{62-64}$, and reactive microglia are thought to create a cytotoxic environment for neurons

427 and exacerbate neurodegneration ${ }^{65-68}$. Here, we provide evidence that neurons participate in this

428 inflammatory signaling as well. Specifically, upon the accumulation of DSBs, neurons are able to 429 engage microglia through the secretion of proinflammatory factors, thus facilitating 
430 neuroinflammation and disease progression. This is a previously undefined role for neurons in the

431 context of neurodegenerative disease, and provides a mechanistic link between genomic fragility

432 and senescence in neurons and microglia activation.

433 Interestingly, the activation of inflammatory signaling in DSB-bearing neurons corresponds with

434 a progressive erosion of cell identity. Using single-nuclei RNA-seq, we find that DSB-bearing

435 neurons engage DNA repair pathways first, but transition to immune gene expression at later stages

436 of genome toxicity. These cells are most enriched for senescent markers, such as Cdkn $1 a$ and $U b b$,

437 rather than neuronal markers. The age-dependent erosion of neuronal identity has been observed

438 in both sporadic and familial Alzheimer's disease patients, and coincides with the activation of a

439 number of biological pathways identified in our study, including DNA damage response, cell-

440 cycle re-entry, and NF-kB signaling ${ }^{31,32}$. Interpreted in the context of the present study, these

441 observations could suggest that the age-associated accumulation of DSBs degrades chromatin

442 integrity, ultimately resulting in the activation of immune signaling that engage microglia.

443 The SASP and canonical antiviral response is activated through the detection of non-endogenous

444 nucleic acids within the cytosol. Thus, it is likely that loss of nuclear and genomic integrity may

445 lead to the release of DNA fragments in DSB-bearing neurons. In fact, we observed increased

446 expression of nucleic acid sensors in DSB-bearing neurons, including cGAS, which plays a

447 significant role in innate immune activation associated with senescence ${ }^{18}$. Nevertheless, many

448 other mechanisms may be at play, including de-repression of transposable elements ${ }^{69}$, epigenetic

$449 \mathrm{drift}^{14}$, and ATM-mediated NFkB transcription ${ }^{19,20}$. There is also evidence that nucleic acids

450 released from mitochondria play roles in neuron immune gene expression ${ }^{70}$. It remains to be

451 addressed whether DSB-induced immune gene expression is a product of age-associated decline 
452 in DNA repair, or whether multiple cellular functions that are also known to decline with age

453 engage immune signaling as a convergent mechanism of neuronal distress.

454 We identified NFkB as a major regulator of immune gene expression in DSB-bearing neurons

455 from both CK-p25 mice, primary neurons, and postmortem human brain. In addition, we found

456 that suppression of NFkB activity through p65 knock down or small molecule inhibition primarily

457 in neurons was sufficient to reduce activated microglia morphology and gene expression. NFkB

458 has been previously identified as a therapeutic target for AD, albeit in the context of microglia and

459 astrocytes $^{71}$. Our results now indicate that this transcription factor plays a pivotal role in DSB-

460 bearing neurons as well. Importantly, in rodent models of learning and memory, NFkB activity in

461 neurons is neuroprotective. Suppression of NFkB in forebrain excitatory neurons impairs spatial

462 learning and neuronal plasticity ${ }^{72}$, and exacerbates cell death following exposure to neurotoxic

463 stimuli $^{73}$. Therefore, the primary role of NFkB may regulate healthy neuronal function in times of

464 homeostasis, but it can also orchestrate immune activation in response to cell stressors.

465 Finally, we identify Cc12 and Cxcl10 as primary signaling molecules secreted from DSB-bearing

466 neurons to recruit and activate microglia. In the CK-p25 model, DSB-bearing neurons are the first

467 cell type to express $\mathrm{Cxc110}$ and $\mathrm{Ccl} 2$. Other cell types express these chemokines at a later

468 timepoint, presumably in response to DSB-bearing neurons. Furthermore, spatial transcriptomics

469 reveal that signatures of reactive microglia are closely associated with $\gamma \mathrm{H} 2 \mathrm{AX}$-positive neurons,

470 suggesting that DSB-bearing neurons are hubs for neuro-immune communication. Notably,

471 increased levels of both of these cytokines are implicated in the pathogenesis of AD, and affect

472 blood brain barrier permeability to aid the infiltration of peripheral monocytes ${ }^{23,43,74,75}$. However,

473 manipulation of either signaling axis seem to have varying effects on AD pathology. For example,

474 Ccr2 deficiency in murine models of AD aggravate amyloid pathology and cognitive decline, but 
$475 \mathrm{Ccl} 2$ overexpression seems to also increase amyloid deposition ${ }^{42,76,77}$. Another study demonstrates

476 that deficiency of the $\mathrm{Cxcl10}$ receptor Cxcr3 reduces amyloid deposition and behavioral deficits ${ }^{78}$.

477 These findings suggest that $\mathrm{Ccl} 2$ and $\mathrm{Cxcl} 10$ are crucial for effective microglia recruitment and

478 activation, but imbalance in these signaling axes have detrimental effects on cognition and

479 pathology clearance.

480 As a whole, we leveraged bulk, single-nuclei, and spatial transcriptomic techniques in parallel with

481 in vitro and in vivo manipulations of NFkB signaling to characterize DSB-bearing neurons and

482 their relationship with microglia in the context of age-associated neurodegenerative disease. We

483 demonstrate that DSB accumulation elicits senescent and antiviral-like signaling in neurons, which

484 recruits and activates microglia in an NFkB-dependent manner. Our data posit that neurons play

485 meaningful roles in neuroinflammation, which historically was largely thought to be driven solely

486 by glial cells. Crucially, this axis of neuron-microglia communication is mediated by DNA damage

487 accumulation in neurons, revealing that two hallmarks of Alzheimer's disease, genome fragility

488 and neuroinflammation, are mechanistically linked. 


\section{Fluorescence-activated nuclei sorting}

491 Frozen cortices were disrupted with a handheld homogenizer in ice-cold PBS with protease inhibitors (cat no. 11836170001, Roche, Basel Switzerland) and RNAse inhibitors (cat no. EO0382, Thermo Fisher Scientific, Waltham MA). Samples were fixed with $1 \%$ paraformaldehyde for 10 minutes at room temperature, then quenched with $2.5 \mathrm{M}$ glycine for 5 minutes. Nuclei were isolated through dounce-homogenization followed by filtration with a 70uM cell strainer (cat no. 21008-952, VWR, Radnor PA). The following antibodies were used to immunotag nuclei: anti-H2A.X-Phosphorylated (Ser139) antibody conjugated to APC (cat no. 613416, BioLegend, San Diego CA), anti-ATM-phospho (Ser1981), antibody conjugated to PE (cat no. 651203 , BioLegend, San Diego CA), anti-NeuN antibody conjugated to Alexa Fluor 488 (cat no. MAB377X, EMD Millipore, Burlington MA), anti-PU.1 antibody conjugated to Alexa 647 (cat no. 2240S, Cell Signaling Technology, Danvers MA), anti-RFP antibody (cat no. 600401-379, Rockland Antibodies and Assays, Gilbertsville, PA), and anti-CaMKII- $\alpha$ (6G9) antibody (cat no. 50049S, Cell signaling Technology, Danvers MA). Antibodies were incubated with nuclei in $1 \% \mathrm{BSA} / \mathrm{PBS}$ at $4{ }^{\circ} \mathrm{C}$ for one hour or overnight. For non-conjugated antibodies, samples were washed once with 1\% BSA/PBS, then resuspended with 1:1000 Alexa Fluor secondary antibody (Thermo Fisher Scientific, Waltham MA) for one hour at $4^{\circ} \mathrm{C}$. Samples were strained through a 40um filter (21008-949, VWR) and stained with DAPI (cat no. D9542, Sigma Aldrich, St. Louis MO) before sorting. Sorting was performed on a FACSAria at the Koch Institute Flow Cytometry Core (BD Biosciences, US). At least 50,000 nuclei of each cell type was collected for RNAsequencing. Nuclei were sorted into $1 \% \mathrm{BSA} / \mathrm{PBS}$, then spun at $2 \mathrm{kG}$ for 15 minutes in a cooled centrifuge (cat no. 97058-916, Epindorf North America) for downstream analysis. For singlenucleus RNA-sequencing, nuclei were not fixed. Single nuclei were sorted into a 96-well plate then transported immediately to the MIT BioMicro Center for library preparation. Flow cytometry analysis was performed using FlowJo software (Ashland, OR).

\section{Human $\gamma \mathrm{H} 2 \mathrm{AX}$ fluorescence-activated nuclei sorting}

516 Frozen temporal cortex tissue samples were homogenized and stained following the protocol described above. A two-week induced CK-p25 mouse sample was used to set a $\gamma \mathrm{H} 2 \mathrm{AX}$ -

518 positive/negative threshold for human nuclei.

\section{Bulk RNA sequencing}

520 For bulk RNA-sequencing of sorted nuclei, samples were treated for 15 minutes with Proteinase $521 \mathrm{~K}$ at $50^{\circ} \mathrm{C}$ and then for 13 minutes at $80^{\circ} \mathrm{C}$. RNA was extracted using Direct-zol RNA MicroPrep 522 kit according to manufacturer's instructions (cat no. R2062, Zymo Research, Irvine CA). Purified 523 RNA samples underwent fragment analysis at the MIT BioMicro Center. Libraries were generated 524 from samples passing quality control (DV200<50\%). Library generation was performed using 525 the SMARTer Stranded Total RNA-Seq Kit v2 - Pico Input Mammalian (cat no. 634412, Takara 
Bio Inc), then submitted to the MIT BioMicro Center for quality control (fragment analysis and qPCR), followed by sequencing. Paired-end sequencing was performed using the Illumina NextSeq500 platform according to standard operating procedures.

529 For bulk RNA sequencing of etoposide-treated primary neurons, cultures were collected in Trizol 530 LS (cat no. 10296028, Thermo Fisher Scientific, Waltham MA). mRNA was purified and extracted using Direct-zol RNA MicroPrep kit according to manufacturer's instructions. RNA was submitted to the MIT BioMicro Center for library preparation and sequencing. Libraries were prepared using NEBNext ${ }^{\circledR}$ Ultra $^{\text {TM }}$ II RNA Library Prep Kit for Illumina ${ }^{\circledR}$ (cat no. E7770, New England Biolabs, Ipswich MA). Single-end sequencing was performed using the Illumina NextSeq500 platform according to standard operating procedures.

\section{Mouse Bulk RNA-seq Read cleaning and bulk RNA-Seq pipeline}

From the paired-end fastq files, the first three nucleotides were trimmed off the second sequencing read using cutadapt version 1.16. TrimGalore version 0.4 .5 was used in paired mode to trim adapters and low-quality portions of reads. Reads were aligned using Salmon version 0.12.0 to mouse genome version GRCm38.94 and human genome version GRCh38.94. For downstream analysis, TPM files from Salmon were imported into R version 3.6.1 using tximport version 1.14.0 with the option to generate estimated counts using abundance estimates scaled up to library size, and additionally scaled using the average transcript length over samples and the library size (countsFromAbundance $=$ lengthScaledTPM).

\section{Mouse Bulk RNA-seq Differential Analysis}

We performed differential analysis using $\mathrm{R}$ version 3.6.1 and DESeq2 version 1.26.0. For each corresponding cell type and condition, we performed differential expression using only the corresponding samples. We selected genes as significant if they met the cutoff threshold (absolute value of the $\log 2$ fold change $>1$, adjusted $p$-value $<0.05$ ).

\section{Mouse Bulk RNA-seq Gene Set Enrichment Analysis}

We used the complete set of RNA-Seq results for each differential analysis for downstream GSEA processing. Genes for which adjusted p-value could not be calculated were excluded. We ran GSEA version 3.0 in ranked list mode with default settings. The gene ontology biological pathways gene sets from Molecular Signatures Database v7.3 (MSigDB) were used for analysis.

\section{Enrichr} occupation in MSigDB gene ontology biological processes containing the keyword "immune." 
This resulted in a Stage 2 immune module comprised of 940 genes. The corresponding Entrez gene symbols were then entered in to the Enrichr website: https://maayanlab.cloud/Enrichr/\#. The resulting transcription factor enrichment data were downloaded as tables from the following transcription factor libraries: ChEA 2016, ENCODE and ChEA Consensus TFs from ChIP-X, TRRUST Transcription Factors 2019, Enrichr Submission TF-Gene Coocurrence, TRANSFAC and JASPAR PWMs, and ENCODE TF ChIP-seq 2015. Data are available in Supplementary Table 1. More information about the Enrichr libraries can be accessed on the Enrichr website.

\section{Smart-seq pipeline}

Single nuclei library preparation was performed by the MIT BioMicro Center using the SMARTSeq ${ }^{\circledR}$ v4 Ultra ${ }^{\circledR}$ Low Input RNA Kit for Sequencing (Takara Bio Inc) according to manufacturer's instruction. Libraries were sequenced on a MiSeq Illumina sequencer according to standard operating procedures.

\section{Read processing.}

574 We aligned 40-bp paired-end reads to the mm10 genome for each of 1,357 single nuclei from 12 mice (64 cells each for $6 \mathrm{CK}$ controls and an average of 162 cells in each of $6 \mathrm{CKp} 25$ mice) using bwa mem (options: -k $15-\mathrm{M}$ ) and filtered out improperly aligned reads and secondary alignments with samtools (options: -f 3 -F 1280). We ran HTSeq-count10 on each cell's filtered bam file to compute the cell's transcriptomic coverage over each gene's exons in vM25 GENCODE gene annotation.

\section{Transgene detection.}

We first aligned 40-bp single-end reads from each sequenced single nucleus separately with the STAR aligner6 against the b37 genome with decoy contigs using a two pass alignment (options: -outFilterMultimapNmax $20 \quad$--alignSJoverhangMin $\quad 8 \quad$--alignSJDBoverhangMin $1 \quad$-alignIntronMin 20 --alignIntronMax 1000000). We then used Picard tools 7 to revert and merge the alignment with unaligned reads and marked duplicates on the merged bam file. We identified and removed alignments on decoy contigs, sorted and fixed NM, MD, and UQ tags with Picard

587 tools, filtered duplicates, unmapped, and non-primary alignment reads, and split reads by Ns in 588 their CIGAR string.

\section{Cell type annotation}

\section{Cell identities}

591 We used SCANPY11 to process and cluster the expression profiles and infer cell identities. We 592 kept only 21,859 protein coding genes detected in at least 3 cells and filtered out 349 cells with 593 less than 100 expressed genes, leaving 1,008 cells over the 12 mice. We used the filtered dataset 
594 to calculate the low dimensional embedding of the cells (UMAP) from the log 1p matrix PCA with $\mathrm{k}=50$ and nearest neighbor graph with $\mathrm{N}=20$ (UMAP default parameters, min_dist=0.2), and clustered it with Leiden clustering (resolution=2), giving 17 preliminary clusters. We then manually assigned clusters based on the following 2-3 major marker genes per class: Excitatory neurons: Camk2a, Gria2, Syt1; Inhibitory neurons: Gad1, Gad2; Astrocytes: Gfap; Microglia: Cd33, Csf1r; Oligodendrocytes: Plp1, Mbp; Oligodendrocyte progenitor cells (OPCs): Bcan; Stage 2: Cdkn1a, Ubb. We merged clusters sharing marker genes to obtain 7 final clusters, defining two broad neuronal subtypes (484 excitatory and 108 inhibitory cells), three glial clusters (50 microglia, 131 oligodendrocytes, and 33 OPCs), 179 Stage 2 cells, and 23 cells with both high read counts and broad, non-specific marker gene expression, likely due to doublets or other sorting artifacts. The doublet cluster was removed from downstream analyses. We also identified a cluster of cells that expressed fewer than 500 genes and had high expression of mitochondrial genes. Due to the questionable quality of these cells, this cluster was also removed from downstream analysis. We further sub-annotated neuronal subtypes from neuronal clusters with distinctive expression in the original Leiden clustering, resulting in four excitatory subclusters (314 Ex0, $91 \mathrm{Ex} 1,32 \mathrm{Ex} 2$, and 47 Ex3 neurons) and two inhibitory subclusters (71 In0 and 37 In1 neurons).

\section{Signature analysis on single-cells}

For each signature, a joint expression value was calculated as the number of reads per 10k reads in each cell coming from all of the signature's genes (genes with adjusted p-values $<0.05$ ). Signature expression was transformed by $\log 1 \mathrm{p}$ and averaged across all cells in a given neuronal subtype and mouse to obtain average signature values for plotting. We used two-sided t-tests to compare the signature expression levels of pairs of excitatory neuronal subtypes (Supplementary Figure 3f-h).

\section{Trajectory analysis}

\section{Pseudotime}

We performed pseudotime analysis using Monocle3 (v0.2.3.0) on the 349 Ex1, Ex2, Ex3, and Stage 2 neuronal populations. We normalized total counts per cell in the read count matrix to the median number of counts, $\log 1 \mathrm{p}$ transformed the matrix, and regressed out the counts per gene using Monocle. We clustered the subsetted data in a new UMAP, clustered cells, learned a trajectory graph, and ordered cells by choosing the initial node as the Ex1 end of the graph to get a pseudotime across the graph. We plot the $\log 1 \mathrm{p}$ read counts across the gene set as well as the generalized additive model (GAM) smoothed fits of each signature's per-cell expression values across pseudotime (Figure 2e,f).

628 We used tradeSeq to detect differentially expressed genes along the pseudotime, by fitting GAMs 
630 test to test for genes with pseudotime-associated expression patterns. We found 530 significant genes. We selected a number of representative genes and plotted their smoothed fit (binned into 100 bins across pseudotime and scaled) and their expression ( $\log 1 \mathrm{p}$ per-cell normalized counts) across the 349 pseudotime-ordered cells. We labeled each gene with the neuronal subtype closest to the pseudotime point with its peak expression (Supplementary Figure 3f).

\section{Ontology enrichment}

636 We created 20 tiled windows of 20 quantiles in width across cellular pseudotime, for each window selected genes whose peak smoothed expression fell into the pseudotime range, and performed GO enrichment on each window's genes using gprofiler, limiting results to terms with fewer than 2,500 genes in GO and Reactome. For visualization, we manually curated 34 representative top enriched terms across all Reactome and GO enrichments (Supplementary Figure 3j).

\section{Human bulk RNA-seq analysis}

642 Paired-end reads were trimmed for adapters using TrimGalore 643 (https://github.com/FelixKrueger/TrimGalore) and aligned to the human genome reference 644 GRCh38/hg38 using the R software package Rsubread (Liao, Smyth, and Shi 2019). Mapped reads 645 were summarized to gene level counts using the featureCounts function of Rsubread, considering 646 the latest RefSeq Reference Genome Annotation for gene reference. Protein coding genes with 647 detected counts in at least 1 sample library were retained and normalized in rpkm units. 648 Normalization and differential expression analysis was performed using the edgeR package 649 (Robinson, McCarthy, and Smyth 2010). All statistical analyses and plots were performed using 650 the statistical programming language R (http://www.r-project.org/). Gene ontology and functional 651 enrichment analyses were performed using gprofiler2 package (Kolberg et al. 2020).

\section{Human bulk gene set activity analysis}

653 Gene set activity for cell type marker genes and gene signatures identified in the Cpk25 mouse 654 model were quantified per human bulk RNAseq sample using Gene Set Variation Analysis 655 (GSVA) (Hänzelmann, Castelo, and Guinney 2013), as implemented in the Bioconductor package 656 GSVA (https://www.bioconductor.org/packages/release/bioc/html/GSVA.html). For cell cell type 657 signature analysis the consensus human brain cell type markers reported in (Mohammadi, Davila658 Velderrain, and Kellis 2020) were used as reference. Differential gene signature activity across 659 conditions $(\gamma \mathrm{H} 2 \mathrm{AX}+/ \mathrm{ctr}, \mathrm{AD} /$ noAD) and/or cell groups ( $\mathrm{NeuN}+\mathrm{NeuN}-)$ was assessed using a 660 two-sided wilcoxon rank sum test.

\section{Human snRNA-seq analysis}

662 Single-nucleus transcriptomic sequencing data from postmortem cortical samples (prefrontal 663 cortex, Brodmann area 10) of 48 subjects with varying levels of AD pathology was obtained from 664 (Mathys et al. 2019). Individual-level celltype expression profiles were computed by averaging for 
each individual the normalized gene-expression profiles across cells of the same cell type. Average profiles were subsequently mean-centered and scaled to compute gene-wise correlation coefficients of gene expression versus individual-level measures of global AD pathology burden reported as part of the ROSMAP cohort. Briefly, global burden of AD pathology is a quantitative summary of AD pathology derived from counts of three AD pathologies: neuritic plaques (n), diffuse plaques (d), and neurofibrillary tangles ( $\mathrm{nft}$ ), as determined by microscopic examination of silver-stained slides (Mathys et al. 2019).

Global consistency between gene signatures observed in CKp25 mice and a neuronal-specific association between gene expression and AD pathology in human tissue was assessed statistically using a nonparametric resampling test. To test whether cell-type specific expression of CKp25 Stage 2 signature genes tends to correlate with pathology in the human brain, a z-score statistic was computed to quantify the deviation of their correlation coefficient rank scores, relative to random expectation. Expected scores were estimated by randomly sampling same-sized gene sets $(\mathrm{n}=1,000$ replicates). This analysis was performed for excitatory neurons, inhibitory neurons, and microglia cells independently.

\section{Stage 1 and 2 signature generation}

681 Stage 1 and Stage 2 gene signatures were curated by performing differential expression analysis on the CK-p25 bulk RNAseq dataset. Stage 1 vs Baseline provided the genes for the Stage 1 signature, and Stage 2 vs Baseline provided the genes for the Stage 2 signature. Only genes which met the cutoff threshold $(\log 2$ fold change $>1$, adjusted $p$-value $<0.05)$ were retained.

\section{Visium spatial transcriptomics library generation}

Mice were transcardially perfused with ice-cold saline, then brains were dissected and flash frozen in OCT. A cryostat was used to generate $10 \mathrm{uM}$ coronal sections of the hippocampus. These sections were applied to $10 \mathrm{X}$ Visium Spatial Gene Expression slides. Sections were immunostained with $\gamma \mathrm{H} 2 \mathrm{AX}$ and DAPI following manufacturer's instructions. Sectioning and staining was performed at the MIT Hope Babette Tang (1983) Histology Core Facility. Sections were imaged immediately after staining using a Olympus FV1200 Laser Scanning Confocal Microscope at the MIT Microscopy Core Facility. Sections were then used to generate 10X Visium Spatial Gene Expression Libraries according to manufacturer's instructions at the MIT BioMicro

694 Center. Libraries were sequenced on a NovaSeq6000 Illumina sequencer according to standard 695 operating procedures.

\section{Visium spatial transcriptomics data processing}

697 Samples were processed using Scanpy 1.7.2. The 7 sample data matrices were merged into one 698 matrix which was then processed. The sample id and location of each capture area of the resulting 699 matrix were saved and used for visualization. Counts were normalized (total count of 10,000 per 700 capture area) and logarithmized (using scanpy's $\log 1 \mathrm{p}$ function). The resulting counts matrix, 
called raw normalized was used for expression visualization and differential expression analysis. For dimension resolution purposes, the raw normalized matrix was further processed: genes that were not characterized as highly variable enough were filtered out (minimum mean of 0.0125 , max mean of 3 , minimum dispersion of 0.5 ), and linear regression was performed to eliminate the effect of covariates (total_counts, and mitochondrial genes percentage). The data was then scaled (standard scaling, max value of 10). Afterwards, PCA was performed, as well as sample-level batch correction, using Harmony. Then, a knn network was constructed for the creation of a UMAP embedding. Clusters were discovered using the leiden algorithm.

\section{Visium immunohistochemistry image processing}

710 Immunostaining images were first processed as shades of gray pictures. The largest

711 autofluorescence artifacts were removed manually. The signal was then amplified and cleaned 712 using a $85 \%$ contrast increase on each image.

713 As the immunochemistry images of the tissue align perfectly to the pictures taken for 10X Visium

714 purposes, calibration was performed to reconstruct the capture areas grid on the immunostaining 715 images. In that image, for each capture area, the mean signal within a circle maximizing image coverage is calculated, and recorded as the DNA-Damage signal. After standard scaling of this variable, a threshold of 0.4 standard deviation was set, to assign a capture areas as positive or negative for DNA damage.

\section{Visium differential expression and Mathys et al., 2017 microglia signature analysis}

Differential expression analysis was performed using Wilcoxon rank sum test, and the resulting pvalues were corrected using Benjamini-Hochberg FDR correction. For GSEA, the package gseapy was used, with 100 permutation and the signal-to-noise method. As input, the raw normalized

\section{RT-qPCR}

725 RNA was extracted from primary tissue cultures using the RNeasy Plus mini kit (cat no. 74136,

726 Qiagen, Hilden Germany). Reverse transcription was performed using Invitrogen SuperScript IV 727 First Strand Synthesis System with Oligo dT primers according to the manufacturer's protocol (cat

728 no. 18091050, Thermo Fisher Scientific, Waltham MA). cDNA was quantified with a NanoDrop 729 spectrophotometer. qPCR was performed using a Bio-Rad CFX-96 quantitative thermal cycler (cat 730 no. 1855195, Bio-Rad, Hercules CA) and SsoFast EvaGreen Supermix (cat no. 1725202, Bio-Rad, 731 Hercules CA). Relative changes in gene expression were determined using the $2^{-\Delta \Delta \mathrm{Ct}}$ method.

732 Cycle numbers for the gene Gapdh or Rpl11 were used for housekeeping Ct values.

\section{Immunofluorescent microscopy}

734 Mice were transcardially perfused with ice-cold PBS, then fixed with ice-cold 4\% 735 paraformaldehyde in PBS. Dissected brains were drop-fixed overnight in 4\% paraformaldehyde in 
PBS at $4^{\circ} \mathrm{C}$. Forebrains were sectioned with a vibrating microtome (Leica BioSystems, Wetzlar Germany) to generate $40 \mathrm{uM}$ coronal slices. Slices were blocked for two hours at room temperature in blocking buffer (10\% NGS, $0.3 \%$ Triton X-100, PBS), then incubated with primary antibody overnight at $4^{\circ} \mathrm{C}$. Slices were washed 3 x 10 minutes with PBS, and Alexa Fluor Secondary antibodies (Thermo Fisher Scientific) were added at a 1:1000 dilution for 1 hour at room temperature. Slices were washed again 3 x 10 minutes with PBS, then stained with DAPI (cat no. D9542, Sigma Aldrich, St. Louis MO) and mounted onto Fisherbrand ${ }^{\mathrm{TM}}$ Superfrost $^{\mathrm{TM}}$ Plus Microscope Slides (cat no. 12-550-15, Thermo Fisher Scientific, Waltham MA) with Fluoromount-G ${ }^{\mathrm{TM}}$ Slide Mounting Medium (cat no. 100502-406, VWR, Radnor PA). once with PBS, then fixed with 4\% paraformaldehyde/PBS for 15 minutes at room temperature. Immunostaining proceeded as described.

Free-floating slices, at 40um thickness from postmortem human brain blocked for 1 hour at room temperature in blocking buffer before being incubated in primary antibodies for 72 hours at $4^{\circ} \mathrm{C}$ with gentle rocking in blocking buffer (Anti-NeuN, Synaptic Systems cat no. 266 004, 1:500; Anti-phospho-Histone H2A.X, Millipore cat no. 05-636, 1:100; Anti-NFkB p65 Invitrogen cat no. 51-0500, 1:300). Samples were then rinsed three times in 1xPBS for 5 minutes and incubated in secondary antibodies (Alexa Fluor-488, 594 or 647, ThermoFisher Scientific; 1:1,000) for 2 hours at room temperature. After 1x PBS rinse, samples were incubated in 1:10,000 Hoechst in PBS (Invitrogen cat no. H3569) followed by 2 minutes in TrueBlack Lipofuscin Autofluorescence Quencher (Biotium cat no. 23007) with 3 subsequent 1x PBS rinses before mounting and imaging.

Mounted samples were imaged with a Zeiss LSM 710 confocal microscope. Images were quantified using ImageJ (NIH Image Analysis) and Imaris (Oxford Instruments). At least two coronal slices were used for each mouse for image quantification. For PHP.eb AAV shp65-RFP cell-type analysis, $40 \mathrm{uM}$ coronal brain sections were stained for RFP and either NeuN, Iba1, GFAP, or Olig2. First, RFP-positive cells were identified in each image. Then, the percent of RFPpositive cells that also had positive immunoreactivity for a given cell-type marker was quantified. GFAP-positive cell number and intensity were calculated using Imaris software (Oxford Instruments, UK).

\section{Primary Neuron Culture}

Cortices were dissected from E15 Swiss-Webster embryos in ice-cold HBSS (cat no. 14175103, Thermo Fisher Scientific, Waltham MA) and dissociated with papain (cat no. LS003126, Worthington Biochemical Corp., Lakewood NJ) and DNAse I (cat no. 10104159001, Roche, Basel Switzerland). Cells were resuspended in plating media (Neurobasal media (cat no. 21103049, Thermo Fisher Scientific, Waltham MA), 1\% Penicillin/Streptomycin Solution (cat no. 400-109, 
773 Cell Counter (cat no. AMQAX1000, Thermo Fisher Scientific, Waltham MA), then plated on poly-

774 D-Lysine-coated 12-well culture dishes, $0.5 \times 10^{\wedge} 6$. Cultures were maintained in $5 \% \mathrm{CO} 2$ at $37^{\circ} \mathrm{C}$ 775 in a cell culture incubator. After allowing four hours for the cells to adhere to the plate, the media 776 was replaced and maintained with neurobasal media supplemented with B-27 (cat no. 17504-044, 777 Invitrogen, Carlsbad CA), 1\% Penicillin/Streptomycin, and 1\% GlutaMAX Supplement (cat no. 778 35050-079, Thermo Fisher Scientific, Waltham MA).

\section{Etoposide Treatment}

780 Primary cortical neuron cultures (DIV11-13) were treated with 1, 5, 10, 25, or 50uM etoposide prepared from 20mM stock (cat no. E1383-250MG, Sigma, St. Louis MO). Cultures were treated for either 3 or 6 hours before collection for downstream experiments. We used two SASP cytokines, $\mathrm{Ccl} 2$ and $\mathrm{Cxcl10}$ (found significantly upregulated in Stage 2 neurons) as biomarkers for activation of immune signaling. Quantitative reverse transcription PCR (RT-qPCR) was used to measure gene expression. A six-hour $50 \mathrm{uM}$ etoposide treatment resulted in a robust induction of compared to other time points and drug concentrations. We also observed that a 50uM etoposide treatment resulted in a marked increase in $\gamma \mathrm{H} 2 \mathrm{AX}$ nuclear intensity compared to control cultures

788 (Figure 2a).

NFkB Activation Inhibitor

Primary neuron cultures were treated with 10uM NF-kappaB Activation Inhibitor VI, benzoxathiole compound from Abcam (cat no. ab145954). Cultures were pre-treated for at least 30 minutes before ETP exposure.

\section{RNAScope In-Situ Hybridization}

794 Fluorescent in-situ hybridization was performed using the RNAscope ${ }^{\circledR}$ Multiplex Fluorescent 795 Reagent Kit v2 according to manufacturer's instructions (cat no. 323100, Advanced Cell 796 Diagnostics, Newark CA). Probes targeting murine Ccl2 (cat no. 311791) and Cxcl10 (cat no. 408921) were purchased from Advanced Cell Diagnostics. Following the RNAscope protocol, slices were stained for $\gamma \mathrm{H} 2 \mathrm{AX}$ (cat no. 05-636, EMD Millipore, Burlington MA). Samples were imaged with a Zeiss LSM 710 confocal microscope at 40x objective. Images were analyzed with ImageJ.

\section{RNAscope Analysis} Process - $>$ "Find Maxima" was used to count mRNA puncta within ROIs. Prominence $>100.00$ with "Strict" setting. The number of $\gamma \mathrm{H} 2 \mathrm{AX}$-positive nuclei with $2 \leq$ maxima were quantified, as well as the number of maxima per $\gamma \mathrm{H} 2 \mathrm{AX}$-positive nucleus. $\gamma \mathrm{H} 2 \mathrm{AX}$-negative nuclei were identified by thresholding for individual nuclei using the DAPI channel, then excluding ROIs that overlapped with $\gamma \mathrm{H} 2 \mathrm{AX}$-positive ROIs. Individual nuclei were identified following the Nuclei 
808 Watershed Separation process described on the ImageJ website:

809 https://imagej.net/Nuclei_Watershed_Separation

810 Brain tissue samples

$811 \quad$ MADRC brain tissue samples

812 Fresh frozen postmortem brain samples were generously provided by the Massachusetts 813 Alzheimer's Disease Research Center. These samples were used for bulk RNA-sequencing. 814 Individuals were selected based on clinical diagnosis and Braak score. The three samples labeled 815 as $\mathrm{AD}$ all had a clinical diagnosis of $\mathrm{AD}$, and a Braak score of VI. The three samples labeled as 816 non-AD did not have a clinical diagnosis of AD, and had Braak scores of I, II and II. Sample 817 metadata is available in Supplementary Table 3.

\section{ROSMAP brain tissue samples}

819 Fixed frozen postmortem brain samples were chosen from the Religious Orders Study and Memory 820 and Aging Project cohort (ROSMAP). ROSMAP is a longitudinal cohort study of ageing and 821 dementia in elderly nuns, brothers and priests. Sample metadata is available in Supplementary 822 Table 4. In-depth description of metadata variables are available at the Rush Alzheimer's Disease 823 Center (RADC) website: https://www.radc.rush.edu/docs/var/variables.htm.

\section{$824 \quad$ P65 knock-down}

825 Custom p65 and scramble shRNA oligos cloned into an AAV backbone (pAV-U6-RFP) were purchased from ViGene Biosciences (Rockville, MD). P65 shRNA sequences were:

1. GATCCGGCAGGCTATCAGTCAGCGCATTGTGCTTATG CGCTGACTGATAGCCTGCTTTTTA

2. GATCCGCGGATTGAGGAGAAACGTAAATGTGCTTTTTA CGTTTCTCCTCAATCCGTTTTTA

3. GATCCGCACCATCAACTATGATGAGTTTGTGCTTAACT CATCATAGTTGATGGTGTTTTTA

4. GATCCGCCTGAGGCTATAACTCGCCTATGTGCTTTAGG CGAGTTATAGCCTCAGGTTTTTA

5. shRNA scramble: GATCCGCAACAAGATGAAGAGCACCAACTCGAGTTGG TGCTCTTCATCTTGTTGTTTTTA

P65 knockdown was confirmed via RT-qPCR in-house. PHP.eB AAV was generated by Janelia Viral Services. PHP.eB AAV shRNA or PBS was delivered retro-orbitally to anesthetized CK-p25 mice, $2 \times 10^{\wedge} 11$. Two weeks after injection, mice were induced by removing doxycycline diet. Following induction, mice were transcardially perfused with ice-cold PBS. One hemisphere was drop-fixed overnight in $4 \%$ paraformaldehyde/PBS at $4^{\circ} \mathrm{C}$ for immunostaining. The other 
844 qPCR. A separate cohort of 7 CK, 5 Scramble, and 6 p65kd CK-p25 mice were used for PU.1845 positive RNA-sequencing, and Ibal and GFAP imaging analyses.

847 CK-p25 double transgenic mice were raised and maintained on a doxycycline diet. All mice were induced by removing doxycycline from their diet to drive the expression of p25-GFP in forebrain excitatory neurons. All mice were induced at 3-4 months old.

\section{Conditioned media and Immunodepletion}

851 Following etoposide treatment, cultures were washed once with PBS. Cultures then recovered in 852 fresh media for 24 hours. Media was collected and spun at 2,000g for 10 minutes to remove cellular 853 debris. Media was stored at $-80^{\circ} \mathrm{C}$ for future experiments. Conditioned media from etoposide854 treated neurons were incubated with $\mathrm{IgG}, \mathrm{Ccl} 2$, or Cxcl10 antibodies (all $40 \mathrm{ug} / \mathrm{mL}$ ) for 4 hours at $8554^{\circ} \mathrm{C}$. Dynabeads ${ }^{\mathrm{TM}}$ Protein $\mathrm{G}$ were added to pull down the antibody complex.

\section{Organotypic Brain Slice Culture}

8578 through 12 week-old $\mathrm{Cx} 3 \mathrm{cr}$-GFP male mice were anesthetized with isoflurane and 858 transcardially-perfused, dissected, and sliced in ice-cold NMDG-cutting solution containing (in $859 \mathrm{mM}$ ): $2.5 \mathrm{KCl}, 0.5 \mathrm{CaCl}$, $10 \mathrm{MgSO} 4,1.25 \mathrm{NaH} 2 \mathrm{PO} 4,20$ HEPES, 2 Thiourea, 5 sodium 860 ascorbate, 3 sodium pyruvate, $92 \mathrm{NMDG}, 30 \mathrm{NaHCO} 3,25 \mathrm{D}-\mathrm{Glucose}, \mathrm{pH} 7.3-7.4$ with HCl. The 861 slicing chamber was bubbled with $95 \% \mathrm{O} 2 / 5 \% \mathrm{CO} 2$, and coronal slices were cut at $250 \mu \mathrm{m}$ 862 thickness using a vibratome (Leica, VT1000s). After the last slice was collected, slices were 863 transferred to a well-plate containing fresh aCSF and placed in an incubator set at $37 \mathrm{C}$ and $95 \%$ $864 \mathrm{O} 2 / 5 \% \mathrm{CO} 2$ for 30 minutes. The aCSF solution contained (in $\mathrm{mM}$ ): $125 \mathrm{NaCl}, 2.5 \mathrm{KCl}, 1.2$ $865 \mathrm{NaH} 2 \mathrm{PO} 4,1.2 \mathrm{MgCl} 2,2.4 \mathrm{CaCl}$, $26 \mathrm{NaHCO}$, 11 D-Glucose. Afterward, 100\% of aCSF was 866 removed and a 1:1 mixture of fresh aCSF and conditioned media was added to the slices and placed 867 back into the incubator for 6 hours. At the completion of the experiment, slices were fixed 868 overnight at $4^{\circ} \mathrm{C}$ with $4 \%$ paraformaldehyde. Slices were then incubated in $30 \%$ glucose overnight 869 at $4^{\circ} \mathrm{C}$. Slices were sub-sectioned into $25 \mathrm{um}$ slices using a cryostat, then cover-slipped for imaging.

\section{$870 \quad$ ELISA}

871 The Mouse MCP1 ELISA Kit and Mouse IP-10 ELISA Kit from Abcam (cat no. ab208979 and 872 ab214563 respectively) were used to quantify $\mathrm{Ccl} 2$ and $\mathrm{Cxcl} 10$ in conditioned media from primary

873 neurons. Assays were quantified on a plate reader and protein concentration was calculated 874 according to manual instructions.

\section{Microglia morphological analysis}

876 Microglia morphology from CK-p25 and acute slice cultures was analyzed according to the 877 protocol described in Young and Morrison, 2018 with minor alterations ${ }^{79}$. Gray Scale Attribute 
878 Filtering (default settings, connectivity: 8) from the MorphoLibJ plug-in version 1.4.1 was used to 879 reduce background noise when thresholding images for skeleton analysis. Following skeleton 880 analysis, Morphological Filters (Operation: Opening, Element: Octagon, Radius (in pixels): 2) 881 from MorphoLibJ was used to quantify soma area. This analysis was performed for both Ibal and 882 Cx3cr1-GFP imaging experiments. 


\section{References}

884 1. Madabhushi, R., Pan, L. \& Tsai, L.-H. DNA Damage and Its Links to Neurodegeneration. Neuron 83, $885 \quad 266-282(2014)$.

2. Martin, L. J. DNA Damage and Repair: Relevance to Mechanisms of Neurodegeneration. $J$. Neuropathol. Exp. Neurol. 67, 377-387 (2008).

3. Hoeijmakers, J. H. J. DNA Damage, Aging, and Cancer. N. Engl. J. Med. 361, 1475-1485 (2009).

4. Lu, T. et al. Gene regulation and DNA damage in the ageing human brain. Nature 429, 883-891 (2004).

5. Adamec, E., Vonsattel, J. P. \& Nixon, R. A. DNA strand breaks in Alzheimer's disease. Brain Res. 849, 67-77 (1999).

6. Lovell, M. A. \& Markesbery, W. R. Oxidative DNA damage in mild cognitive impairment and latestage Alzheimer's disease. Nucleic Acids Res. 35, 7497-7504 (2007).

7. Jacobsen, E., Beach, T., Shen, Y., Li, R. \& Chang, Y. Deficiency of the Mre11 DNA repair complex in Alzheimer's disease brains. Mol. Brain Res. 128, 1-7 (2004).

8. Shanbhag, N. M. et al. Early neuronal accumulation of DNA double strand breaks in Alzheimer's disease. Acta Neuropathol. Commun. 7, 77 (2019). Alzheimer disease phenotypes. Nucleic Acids Res. 43, 943-959 (2015). neurons. Nat. Neurosci. 16, 1008-1015 (2013). 
904 12. Suberbielle, E. et al. DNA repair factor BRCA1 depletion occurs in Alzheimer brains and impairs 905 cognitive function in mice. Nat. Commun. 6, 8897 (2015).

906 13. Suberbielle, E. et al. Physiologic brain activity causes DNA double-strand breaks in neurons, with 907 exacerbation by amyloid-ß. Nat. Neurosci. 16, 613-621 (2013).

908 14. Hayano, M. et al. DNA Break-Induced Epigenetic Drift as a Cause of Mammalian Aging.

909 https://papers.ssrn.com/abstract=3466338 (2019) doi:10.2139/ssrn.3466338.

910 15. Wu, W. et al. Neuronal enhancers are hotspots for DNA single-strand break repair. Nature 593, 440-

$911444(2021)$.

912 16. Reid, D. A. et al. Incorporation of a nucleoside analog maps genome repair sites in postmitotic 913 human neurons. Science 372, 91-94 (2021).

914 17. Härtlova, A. et al. DNA Damage Primes the Type I Interferon System via the Cytosolic DNA Sensor 915 STING to Promote Anti-Microbial Innate Immunity. Immunity 42, 332-343 (2015).

916 18. Dou, Z. et al. Cytoplasmic chromatin triggers inflammation in senescence and cancer. Nature 550, $917 \quad 402-406(2017)$.

918 19. Janssens, S. \& Tschopp, J. Signals from within: the DNA-damage-induced NF- $\kappa$ B response. Cell 919 Death Differ. 13, 773-784 (2006).

920 20. McCool, K. W. \& Miyamoto, S. DNA damage-dependent NF-кB activation: NEMO turns nuclear $921 \quad$ signaling inside out. Immunol. Rev. 246, 311-326 (2012).

922 21. Rodier, F. et al. Persistent DNA damage signalling triggers senescence-associated inflammatory 923 cytokine secretion. Nat. Cell Biol. 11, 973-979 (2009).

924 22. Chien, Y. et al. Control of the senescence-associated secretory phenotype by NF- B promotes 925 senescence and enhances chemosensitivity. Genes Dev. 25, 2125-2136 (2011). 
23. Klein, R. S. et al. Neuronal CXCL10 Directs CD8+ T-Cell Recruitment and Control of West Nile Virus Encephalitis. J. Virol. 79, 11457-11466 (2005).

24. Préhaud, C., Mégret, F., Lafage, M. \& Lafon, M. Virus Infection Switches TLR-3-Positive Human Neurons To Become Strong Producers of Beta Interferon. J. Virol. 79, 12893-12904 (2005).

25. Cruz, J. C., Tseng, H.-C., Goldman, J. A., Shih, H. \& Tsai, L.-H. Aberrant Cdk5 Activation by p25 Triggers Pathological Events Leading to Neurodegeneration and Neurofibrillary Tangles. Neuron 40,

26. Mathys, H. et al. Temporal Tracking of Microglia Activation in Neurodegeneration at Single-Cell

$934 \quad$ Resolution. Cell Rep. 21, 366-380 (2017).

27. Cruz, J. C. et al. p25/Cyclin-Dependent Kinase 5 Induces Production and Intraneuronal Accumulation of Amyloid beta In Vivo. J. Neurosci. 26, 10536-10541 (2006). Prolonged Expression of p25 in Synaptic Plasticity and Hippocampus-Dependent Memory. Neuron

29. Gjoneska, E. et al. Conserved epigenomic signals in mice and humans reveal immune basis of

$941 \quad$ Alzheimer's disease. Nature 518, 365-369 (2015).

942 30. Mah, L.-J., El-Osta, A. \& Karagiannis, T. C. $\gamma \mathrm{H} 2 \mathrm{AX}$ : a sensitive molecular marker of DNA damage 943 and repair. Leukemia 24, 679-686 (2010).

944 31. Caldwell, A. B. et al. Dedifferentiation and neuronal repression define familial Alzheimer's disease. 945 Sci. Adv. 6, eaba5933 (2020).

946 32. Mertens, J. et al. Age-dependent instability of mature neuronal fate in induced neurons from Alzheimer's patients. Cell Stem Cell (2021) doi:10.1016/j.stem.2021.04.004. 
33. Yousef, A. et al. Neuron loss and degeneration in the progression of TDP-43 in frontotemporal lobar degeneration. Acta Neuropathol. Commun. 5, 68 (2017).

34. Ünal-Çevik, I., Kılınç, M., Gürsoy-Özdemir, Y., Gurer, G. \& Dalkara, T. Loss of NeuN immunoreactivity after cerebral ischemia does not indicate neuronal cell loss: a cautionary note.

35. McPhail, L. T., McBride, C. B., McGraw, J., Steeves, J. D. \& Tetzlaff, W. Axotomy abolishes NeuN Brain Res. 1015, 169-174 (2004).

36. Wu, K.-L. et al. Loss of Neuronal Protein Expression in Mouse Hippocampus After Irradiation. $J$. Neuropathol. Exp. Neurol. 69, 272-280 (2010).

37. Collombet, J.-M. et al. Early reduction of NeuN antigenicity induced by soman poisoning in mice can be used to predict delayed neuronal degeneration in the hippocampus. Neurosci. Lett. 398, 337-342

38. Subramanian, A. et al. Gene set enrichment analysis: A knowledge-based approach for interpreting genome-wide expression profiles. Proc. Natl. Acad. Sci. 102, 15545-15550 (2005).

39. Yang, Y., Geldmacher, D. S. \& Herrup, K. DNA Replication Precedes Neuronal Cell Death in Alzheimer's Disease. J. Neurosci. 21, 2661-2668 (2001).

40. Busser, J., Geldmacher, D. S. \& Herrup, K. Ectopic Cell Cycle Proteins Predict the Sites of Neuronal Cell Death in Alzheimer's Disease Brain. J. Neurosci. 18, 2801-2807 (1998). Inflammation Observed in Alzheimer's Disease. Brain Pathol. 19, 392-398 (2009). 
970 43. Chai, Q., She, R., Huang, Y. \& Fu, Z. F. Expression of Neuronal CXCL10 Induced by Rabies Virus 971 Infection Initiates Infiltration of Inflammatory Cells, Production of Chemokines and Cytokines, and 972 Enhancement of Blood-Brain Barrier Permeability. J. Virol. 89, 870-876 (2014).

973 44. Conductier, G., Blondeau, N., Guyon, A., Nahon, J.-L. \& Rovère, C. The role of monocyte 974 chemoattractant protein MCP1/CCL2 in neuroinflammatory diseases. J. Neuroimmunol. 224, 93-100 975 (2010).

976 45. Chen, E. Y. et al. Enrichr: interactive and collaborative HTML5 gene list enrichment analysis tool. BMC Bioinformatics 14, 128 (2013).

978 46. Kuleshov, M. V. et al. Enrichr: a comprehensive gene set enrichment analysis web server 2016 $979 \quad$ update. Nucleic Acids Res. 44, W90-97 (2016).

47. Xie, Z. et al. Gene Set Knowledge Discovery with Enrichr. Curr. Protoc. 1, e90 (2021).

981 48. Trapnell, C. et al. The dynamics and regulators of cell fate decisions are revealed by pseudotemporal ordering of single cells. Nat. Biotechnol. 32, 381-386 (2014).

983 49. Qiu, X. et al. Reversed graph embedding resolves complex single-cell trajectories. Nat. Methods 14, $984 \quad 979-982(2017)$.

50. Cao, J. et al. The single-cell transcriptional landscape of mammalian organogenesis. Nature 566, $986 \quad 496-502(2019)$.

987 51. Wang, C. et al. Gain of toxic apolipoprotein E4 effects in human iPSC-derived neurons is 988 ameliorated by a small-molecule structure corrector. Nat. Med. 24, 647-657 (2018).

989 52. Dunphy, G. et al. Non-canonical Activation of the DNA Sensing Adaptor STING by ATM and IFI16 990 Mediates NF-кB Signaling after Nuclear DNA Damage. Mol. Cell 71, 745-760.e5 (2018). 
991 53. Mathys, H. et al. Single-cell transcriptomic analysis of Alzheimer's disease. Nature 570, 332-337

992 (2019).

993 54. Variable Details | RADC.

994 https://www.radc.rush.edu/docs/var/detail.htm?category=Pathology\&subcategory=Alzheimer\%27s $+\mathrm{d}$

995 isease\&variable $=$ gpath.

996 55. Chan, K. Y. et al. Engineered AAVs for efficient noninvasive gene delivery to the central and

997 peripheral nervous systems. Nat. Neurosci. 20, 1172-1179 (2017).

998 56. Chang, A. L. et al. CCL2 Produced by the Glioma Microenvironment Is Essential for the Recruitment 999 of Regulatory T Cells and Myeloid-Derived Suppressor Cells. Cancer Res. 76, 5671-5682 (2016).

57. Semple, B. D., Bye, N., Rancan, M., Ziebell, J. M. \& Morganti-Kossmann, M. C. Role of CCL2 (MCP-1) in traumatic brain injury (TBI): evidence from severe TBI patients and CCL2-/- mice. $J$. Cereb. Blood Flow Metab. Off. J. Int. Soc. Cereb. Blood Flow Metab. 30, 769-782 (2010).

1003 58. Galimberti, D. et al. Serum MCP-1 levels are increased in mild cognitive impairment and mild 1004 Alzheimer's disease. Neurobiol. Aging 27, 1763-1768 (2006).

1005 59. Bradburn, S. et al. Dysregulation of C-X-C motif ligand 10 during aging and association with 1006 cognitive performance. Neurobiol. Aging 63, 54-64 (2018). Alzheimer's Disease. Cell 153, 707-720 (2013). Alzheimer's Disease. Cell 169, 1276-1290.e17 (2017). 
1011

1012

1013

1014

1015

1016

1017

1018

1019

1020

1021

1022

1023

1024

1025

1026

1027

1028

1029

1030

1031

1032

62. Alzheimer Disease Genetics Consortium (ADGC), et al. Genetic meta-analysis of diagnosed Alzheimer's disease identifies new risk loci and implicates $A \beta$, tau, immunity and lipid processing. Nat. Genet. 51, 414-430 (2019).

63. Nott, A. et al. Brain cell type-specific enhancer-promoter interactome maps and disease - risk association. Science 366, 1134-1139 (2019).

64. Ramamurthy, E. et al. Cell type-specific histone acetylation profiling of Alzheimer's Disease subjects and integration with genetics. 2020.03.26.010330

https://www.biorxiv.org/content/10.1101/2020.03.26.010330v1 (2020)

doi:10.1101/2020.03.26.010330.

65. Shi, Y. et al. Microglia drive APOE-dependent neurodegeneration in a tauopathy mouse model. $J$. Exp. Med. 216, 2546-2561 (2019).

66. Spangenberg, E. et al. Sustained microglial depletion with CSF1R inhibitor impairs parenchymal plaque development in an Alzheimer's disease model. Nat. Commun. 10, 3758 (2019).

67. Spangenberg, E. E. et al. Eliminating microglia in Alzheimer's mice prevents neuronal loss without modulating amyloid- $\beta$ pathology. Brain 139, 1265-1281 (2016).

68. Song, W. M. \& Colonna, M. The identity and function of microglia in neurodegeneration. Nat. Immunol. 19, 1048-1058 (2018).

69. Simon, M. et al. LINE1 Derepression in Aged Wild-Type and SIRT6-Deficient Mice Drives Inflammation. Cell Metab. 29, 871-885.e5 (2019).

70. Lee, H. et al. Cell Type-Specific Transcriptomics Reveals that Mutant Huntingtin Leads to Mitochondrial RNA Release and Neuronal Innate Immune Activation. Neuron 107, 891-908.e8 (2020). 
71. Wyss-Coray, T. \& Rogers, J. Inflammation in Alzheimer Disease-A Brief Review of the Basic Science and Clinical Literature. Cold Spring Harb. Perspect. Med. 2, (2012).

72. Kaltschmidt, B. et al. NF-kappaB regulates spatial memory formation and synaptic plasticity through protein kinase A/CREB signaling. Mol. Cell. Biol. 26, 2936-2946 (2006).

73. Fridmacher, V. et al. Forebrain-Specific Neuronal Inhibition of Nuclear Factor- $\kappa B$ Activity Leads to

1038 Loss of Neuroprotection. J. Neurosci. 23, 9403-9408 (2003).

74. Mahad, D. et al. Modulating CCR2 and CCL2 at the blood-brain barrier: relevance for multiple sclerosis pathogenesis. Brain 129, 212-223 (2006).

75. Dimitrijevic, O. B., Stamatovic, S. M., Keep, R. F. \& Andjelkovic, A. V. Effects of the Chemokine CCL2 on Blood-Brain Barrier Permeability during Ischemia-Reperfusion Injury. J. Cereb. Blood Flow Metab. 26, 797-810 (2006).

76. Naert, G. \& Rivest, S. CC Chemokine Receptor 2 Deficiency Aggravates Cognitive Impairments and 1045 Amyloid Pathology in a Transgenic Mouse Model of Alzheimer's Disease. J. Neurosci. 31, 6208$1046 \quad 6220(2011)$.

1047 77. Yamamoto, M. et al. Overexpression of Monocyte Chemotactic Protein-1/CCL2 in $\beta$-Amyloid 1048 Precursor Protein Transgenic Mice Show Accelerated Diffuse $\beta$-Amyloid Deposition. Am. J. Pathol. $1049 \quad \mathbf{1 6 6}, 1475-1485(2005)$.

78. Krauthausen, M. et al. CXCR3 promotes plaque formation and behavioral deficits in an Alzheimer's disease model. J. Clin. Invest. 125, 365-378 (2015). 
Methods References

1056 1. Kolberg, Liis, Uku Raudvere, Ivan Kuzmin, Jaak Vilo, and Hedi Peterson. "Gprofiler2 -- an R 1057 Package for Gene List Functional Enrichment Analysis and Namespace Conversion Toolset 1058 g:Profiler.” F1000Research 9 (November 17, 2020): 709.

1059 https://doi.org/10.12688/f1000research.24956.2.

1060 2. Liao, Yang, Gordon K. Smyth, and Wei Shi. "The R Package Rsubread Is Easier, Faster, 1061 Cheaper and Better for Alignment and Quantification of RNA Sequencing Reads.” Nucleic 1062 Acids Research 47, no. 8 (May 7, 2019): e47. https://doi.org/10.1093/nar/gkz114.

1063 3. Mohammadi, Shahin, Jose Davila-Velderrain, and Manolis Kellis. "A Multiresolution 1064 Framework to Characterize Single-Cell State Landscapes.” Nature Communications 11, no. 1 1065 (October 26, 2020): 5399. https://doi.org/10.1038/s41467-020-18416-6.

1066 4. Robinson, Mark D., Davis J. McCarthy, and Gordon K. Smyth. "EdgeR: A Bioconductor 1067 Package for Differential Expression Analysis of Digital Gene Expression Data.”

1068 Bioinformatics (Oxford, England) 26, no. 1 (January 1, 2010): 139-40.

1069 https://doi.org/10.1093/bioinformatics/btp616.

1070 5. Bennett, David A., Aron S. Buchman, Patricia A. Boyle, Lisa L. Barnes, Robert S. Wilson, 1071 and Julie A. Schneider. "Religious Orders Study and Rush Memory and Aging Project." 1072 Journal of Alzheimer's Disease: JAD 64, no. s1 (2018): S161-89.

1073 https://doi.org/10.3233/JAD-179939.

1074 6. Swiech, Lukasz, Matthias Heidenreich, Abhishek Banerjee, Naomi Habib, Yinqing Li, John 1075 Trombetta, Mriganka Sur, and Feng Zhang. "In Vivo Interrogation of Gene Function in the 1076 Mammalian Brain Using CRISPR-Cas9.” Nature Biotechnology 33, no. 1 (January 2015): 1077 102-6. https://doi.org/10.1038/nbt.3055. 
1078 7. Dobin, Alexander, Carrie A. Davis, Felix Schlesinger, Jorg Drenkow, Chris Zaleski, Sonali 1079 Jha, Philippe Batut, Mark Chaisson, and Thomas R. Gingeras. "STAR: Ultrafast Universal 1080 RNA-Seq Aligner.” Bioinformatics (Oxford, England) 29, no. 1 (January 1, 2013): 15-21. $1081 \quad$ https://doi.org/10.1093/bioinformatics/bts635.

1082 8. Anders, Simon, Paul Theodor Pyl, and Wolfgang Huber. "HTSeq--a Python Framework to 1083 Work with High-Throughput Sequencing Data.” Bioinformatics (Oxford, England) 31, no. 2 1084 (January 15, 2015): 166-69. https://doi.org/10.1093/bioinformatics/btu638.

1085 9. Wolf, F. Alexander, Philipp Angerer, and Fabian J. Theis. "SCANPY: Large-Scale Single1086 Cell Gene Expression Data Analysis.” Genome Biology 19, no. 1 (February 6, 2018): 15. 1087 https://doi.org/10.1186/s13059-017-1382-0.

1088 10. De Jager, Philip L., Yiyi Ma, Cristin McCabe, Jishu Xu, Badri N. Vardarajan, Daniel Felsky, 1089 Hans-Ulrich Klein, et al. "A Multi-Omic Atlas of the Human Frontal Cortex for Aging and 1090 Alzheimer's Disease Research.”Scientific Data 5, no. 1 (August 7, 2018): 180142. $1091 \quad$ https://doi.org/10.1038/sdata.2018.142.

1092 11. Storey, John D., Andrew J. Bass, Alan Dabney, David Robinson, and Gregory Warnes.

1093 Qvalue: Q-Value Estimation for False Discovery Rate Control (version 2.22.0). Bioconductor 1094 version: Release (3.12), 2021. https://doi.org/10.18129/B9.bioc.qvalue.

1095 12. Raudvere, Uku, Liis Kolberg, Ivan Kuzmin, Tambet Arak, Priit Adler, Hedi Peterson, and 1096 Jaak Vilo. “G:Profiler: A Web Server for Functional Enrichment Analysis and Conversions of 1097 Gene Lists (2019 Update).” Nucleic Acids Research 47, no. W1 (July 2, 2019): W191-98. 1098 https://doi.org/10.1093/nar/gkz369. 
1099 13. Jassal, Bijay, Lisa Matthews, Guilherme Viteri, Chuqiao Gong, Pascual Lorente, Antonio

1100 Fabregat, Konstantinos Sidiropoulos, et al. “The Reactome Pathway Knowledgebase.” Nucleic

1101 Acids Research 48, no. D1 (January 8, 2020): D498-503. https://doi.org/10.1093/nar/gkz1031.

1102 14. Ligtenberg, W. "Reactome.Db: A Set of Annotation Maps for Reactome Assembled Using

1103 Data from Reactome.” Bioconductor, 2019. http://bioconductor.org/packages/reactome.db/.

1104 15. Mathys, Hansruedi, Jose Davila-Velderrain, Zhuyu Peng, Fan Gao, Shahin Mohammadi,

1105 Jennie Z. Young, Madhvi Menon, et al. "Single-Cell Transcriptomic Analysis of Alzheimer's

1106 Disease.” Nature 570, no. 7761 (June 2019): 332-37. https://doi.org/10.1038/s41586-019-

$1107 \quad \underline{1195-2 .}$

\section{Acknowledgements}

1109 We thank M. Saturno-Condon, M. Jennings, M. Griffin, and G. Paradis from the Swanson

1110 Biotechnology Center Flow Cytometry Facility for assistance with FANS. We thank S. Levine, N.

1111 Kamelamela, and A. Hendricks from the MIT BioMicro Center for assistance with RNA

1112 sequencing and library preparation. We thank K. Cormier from the MIT Hope Babetter Tang

1113 (1983) Histology Core Facility and J. Khun from the MIT Microscopy Core Facility for assistance

1114 with Visium Spatial Gene Expression sample preparation. This work was supported by NIH grants

1115 AG054012, AG058002, AG062377, NS110453, NS115064, AG067151, AG062335, MH109978,

1116 MH119509, and HG008155 (to M.K. and L.H.T), 5 R01 NS102730-03 (to L.H.T), and CureAlz

1117 CIRCUITS, and the Glenn Foundation for Medical Research. This work was also supported by

1118 NIH fellowship 5F31NS113464-02 to G.W. V.D. is supported by the AARF-19-618751 grant from

1119 the Alzheimer's Association. M.B.V. is supported by HHMI Hanna H. Gray Fellowship. C.A.B.

1120 is supported by the NIH training grant GM087237. 


\section{Figure Legends}

1122

1123

1124

1125

1126

1127

1128

1129

1130

1131

1132

1133

1134

1135

1136

1137

1138

1139

1140

1141

1142

1143

1144

1145

1146

1147

1148

1149

1150

1151

1152

1153

1154

1155

1156

Figure 1. Neurons marked by DNA DSBs activate inflammatory signaling at early stages of neurodegeneration.

a. Flow cytometry dot plots of $\gamma \mathrm{H} 2 \mathrm{AX}+$ nuclei from $\mathrm{CK}$ and $\mathrm{CK}-\mathrm{p} 25$ cortex. $\gamma \mathrm{H} 2 \mathrm{AX}+$ are highlighted in turquoise, and percent total population is indicated above the gating box.

b. CK-p25 mice were taken off dox for 1 through 6 weeks, then cortical nuclei were extracted and stained for $\gamma \mathrm{H} 2 \mathrm{AX}$. Each datapoint represents percent $\gamma \mathrm{H} 2 \mathrm{AX}+$ nuclei from one mouse cortex.

c. RNA seq workflow for the gated populations. Bulk RNA-seq: Cortices were extracted from CK and CK-p25 mice ( $\mathrm{n}=2$ per genotype for bulk RNA-seq, 2-week timepoint only). Tissue was homogenized, then nuclei were extracted and stained for NeuN and $\gamma \mathrm{H} 2 \mathrm{AX}$. 50,000 nuclei were sorted from each gated population. snRNA-seq: Cortices were extracted from CK and CK-p25 mice ( $\mathrm{n}=3$ per genotype $\times$ timepoint for SMART-seq, 1-week and 2-week timepoints). Tissue was homogenized, then nuclei were extracted and stained for NeuN and $\gamma \mathrm{H} 2 \mathrm{AX} .48$ nuclei were sorted for each gated CK-p25 population per mouse, and 32 nuclei were sorted for each gated CK population per mouse.

d. Differential gene ontology terms from gene set enrichment analysis (GSEA) of bulk RNAseq data. Left column depicts GSEA results from Stage 1 vs. CK-p25 Baseline contrast. Right column depicts GSEA results from Stage 2 vs. CK-p25 Baseline contrast. Color indicates normalized enrichment score (NES). Size indicates false discovery rate (FDR).

e. Heatmap of differentially expressed genes belonging to inflammatory gene sets from bulk RNA-seq data. Each column represents one mouse. Gene sets are organized by biological function.

f. (left): Representative images of Ccl2 RNAscope combined with $\gamma \mathrm{H} 2 \mathrm{AX}$ immunofluorescent staining. Imaging was performed on $1 \mathrm{wk}$ and $2 \mathrm{wk}$ CK-p25 cortex. (right): Quantification of number of $\gamma \mathrm{H} 2 \mathrm{AX}+$ and $\gamma \mathrm{H} 2 \mathrm{AX}$ - cells with $2 \leq \mathrm{Cc} 22$ puncta. Each datapoint respresents average $\% \mathrm{Ccl} 2$-positive cells in one image from one mouse. 4-3 images were taken per mouse. (Ccl2 1wk n=4, Cc12 2wk n=5)

g. Representative image of p65 immunostaining in CK-p25 cortex at two-week timepoint. White arrowheads indicate $\gamma \mathrm{H} 2 \mathrm{AX}+$ nuclei with nuclear p65 immunoreactivity. Quantification of p65 mean intensity for $\gamma \mathrm{H} 2 \mathrm{AX}+$ and $\gamma \mathrm{H} 2 \mathrm{AX}$ - nuclei. Each data point represents the average $\mathrm{p} 65$ nuclear mean gray value of 20-60 nuclei from one mouse.

h. UMAP of gated populations from CK and CK-p25 cortex at 1-week and 2-week timepoints. Colors indicate cell type annotation.

i. Marker gene expression for each cell type cluster. Columns represent 985 individual cells ordered by cell type cluster. 
1157 j. Trajectory analysis of Ex0,1,2,3, and Stage 2 neurons. Cells from each cluster (indicated by 1158 color annotation) are ordered across pseudotime (indicated by color gradient).

1159 k. Trajectory analysis of Ex0,1,2,3, and Stage 2 neurons. Smoothened gene signature expression 1160 across pseudotime. Stage 1=Significantly upregulated genes from Stage 1 vs. Baseline contrast. 1161 Stage $2=$ Significantly upregulated genes from Stage 2 vs. Baseline contrast. Immune= Genes 1162 belonging to immune gene ontologies that were significantly upregulated in Stage 2 neurons. $1163 \operatorname{padj}<0.05, \log 2$ fold-change $\geq 1.0$.

1164 Error bars represent standard error of mean (S.E.M.); **** $\mathrm{P}<0.0001, * * * \mathrm{P}<0.001, * * \mathrm{P}<0.01$, $1165 * \mathrm{P}<0.05$; One-way ANOVA with Tukey's test for multiple comparisons (b,f). Two-way ANOVA 1166 followed by Sidak's test for multiple comparisons (g). Data are pooled from 4 independent 1167 experiments (b). Data are representative of two independent experiments (f,g). 
Figure 2. Induction of DNA DSBs is sufficient to elicit immune pathway signaling in neuron primary culture.

a. Top: Representative images of NeuN and $\gamma \mathrm{H} 2 \mathrm{AX}$ immunostaining in ETP and vehicle-treated primary cultures. $\gamma \mathrm{H} 2 \mathrm{AX}$ immunoreactivity is quantified for each condition. Each data point represents $\gamma \mathrm{H} 2 \mathrm{AX}$ mean gray value for one nucleus in the representative image. Bottom: Representative images of p65 immunostaining in ETP and vehicle-treated primary cultures. p65 immunoreactivity is quantified for each condition. Each data point represents p65 mean gray value for one nucleus in the representative image.

b. Schematic of etoposide (ETP) treatment. DIV13 neuron primary culture were treated with either 50uM ETP or vehicle control (DMSO) for 6 hours. mRNA was extracted from the cultures and 1178 sequenced.

1179 c. Differential gene ontology terms identified through gene set enrichment analysis (GSEA) from 1180 ETP vs. DMSO contrast.

d. Heatmap of Stage 1 and Stage 2 signature enrichment (from bulk RNAseq) in DMSO and ETPtreated neurons.

e. Venn diagram of significantly upregulated protein-coding genes from ETP-treated neurons and Stage 1 and Stage 2 gene signatures. Percentages are in reference to the total number of unique genes from all three gene sets. Example genes are shown to the right. Genes overlapping in ETP and Stage 2 are in turquoise. Genes overlapping in ETP, Stage 2, and Stage 1 are in magenta. The area of the circles are in proportion to the size of the gene sets.

f. Representative images of $\mathrm{NeuN}$ and $\gamma \mathrm{H} 2 \mathrm{AX}$ immunostaining from 10 Gy and 0 Gy $\mathrm{x}$-ray irradiation treated primary neurons.

g. qRT-PCR of Cdkn1a, Ccl2, and Cxcl10 in 10 Gy irradiated primary neurons. Each datapoint represents one biological replicate.

1192 Error bars represent standard error of mean (S.E.M.); ****P $<0.0001$, ***P $<0.001$. Student's Ttest $(a, g)$. Data are representative of 3 independent experiments (a). Data are representative of 2 independent experiments $(\mathrm{g})$. 
Figure 3. Inflammatory signaling in DSB-bearing neurons is positively correlated with Alzheimer's disease pathology.

1197 a. Schematic of the Stage 2 signature analysis in the snRNA-seq dataset from Mathys et al., 2019.

b. Quantification of Stage 2 signature correlation with global pathology in celltype clusters from Mathys et al., 2019. It was tested if stage 2 genes were significantly and positively correlated with the global pathology metric for each major celltype. The - $\log 10 \mathrm{p}$-value for these tests are shown in the histogram. The dashed line indicates a p-value of 0.01 after Bonferroni correction for multiple testing. Excitatory neurons (Ex), Inhibitory neurons (In), Astrocytes (Ast), Oligodendrocytes (Oli), Oligodendrocyte precursor cells (Opc), Microglia (Mic).

c. Stage 2 signature genes ranked by their correlation with global pathology in excitatory neurons. Stage 2 genes with positive correlation are shown with red circles.

d. Gene ontology enrichment of Stage 2 signature genes positively correlated with global pathology.

e. Schematic of $\gamma \mathrm{H} 2 \mathrm{AX}+$ nuclei sorting from $\mathrm{AD}$ and non-AD brain tissue.

f. Heatmap of Stage 1 and Stage 2 signature enrichment in $\gamma \mathrm{H} 2 \mathrm{AX}+$ and $\gamma \mathrm{H} 2 \mathrm{AX}$ - human NeuN + nuclei.

1211 g-i. Quantification of Stage 1 and Stage 2 signature enrichment in $\gamma \mathrm{H} 2 \mathrm{AX}+$ and $\gamma \mathrm{H} 2 \mathrm{AX}-$ human NeuN+ nuclei samples by FANS gate $(g)$, and FANS gate and disease status $(\mathrm{h}, \mathrm{i})$.

1213 j. Representative image of $\gamma \mathrm{H} 2 \mathrm{AX}$, p65, and NeuN in the AD brain. Left: two NeuN-positive nuclei are outlined (white dashed line). Right: magnification of the two outlined nuclei. Top nucleus (A) represents low $\gamma \mathrm{H} 2 \mathrm{AX}$ burden, bottom nucleus (B) represents high $\gamma \mathrm{H} 2 \mathrm{AX}$ burden.

1216 k. Quantification of p65 nuclear enrichment in low and high $\gamma \mathrm{H} 2 \mathrm{AX}$-burdened neurons. Each dot represents the average of 23-41 NeuN+ nuclei per individual.

1218 Error bars represent standard error of mean (S.E.M.); **** $\mathrm{P}<0.0001, * * * \mathrm{P}<0.001, * * \mathrm{P}<0.01$, $* \mathrm{P}<0.05$, ns not significant. T-test $(\mathrm{k})$. Wilcoxon test (g-i). 
Figure 4. Immune signaling in neurons recruits and activates microglia.

1221 a. Schematic of spatial transcriptomics experiment. CK $(n=3)$ and CK-p25 $(n=4)$ were induced for two weeks. Coronal brain sections were stained and imaged for $\gamma \mathrm{H} 2 \mathrm{AX}$, then sequenced.

1223 b. UMAP of capture areas from all samples. Leiden clusters are indicated by color and number. Each dot represents one capture area.

1225

c. Leiden clusters superimposed onto a CK-p25 brain slice used for spatial transcriptomics.

d. UMAP indicating the density of $\gamma \mathrm{H} 2 \mathrm{AX}$-positive capture areas.

e. $\gamma \mathrm{H} 2 \mathrm{AX}$-positive capture areas identified in one CK-p25 brain slice.

f. Spatial clusters, $\gamma \mathrm{H} 2 \mathrm{AX}$-positive capture areas, and reactive microglia signature gene expression in one CK-p25 sample.

1230 g. Schematic of neuronal p65 knock-down experiment. CK-p25 mice receive retro-orbital injections of scramble shRNA-RFP AAV or shp65-RFP AAV. CK mice received retro-orbital injections of PBS. Mice were allowed to recover for two weeks before being taken off dox. Brains were collected for subsequent analysis at the two week timepoint.

h. qRT-PCR of immune genes in sorted $\gamma \mathrm{H} 2 \mathrm{AX}+$ nuclei.

i. Representative images of Iba1 immunostaining in CK, Scramble, and p65kd cortex.

j. Quantification of (left to right): Iba1+ soma area, number Iba1+ per image, average branch length per Iba1+ cell, and number end-points per Iba1+ cell. Each data point represents one image. Two images were taken per mouse.

k. Heat map of differentially expressed genes from P65 vs. Scramble contrast. Each column represents one mouse.

1241 I. Upregulated and downregulated gene ontology (biological pathway) terms identified through gene set enrichment analysis (GSEA) from p65 kd vs. scramble contrast.

m. Heatmap of significantly upregulated and downregulated genes in Pu.1+ nuclei from p65 kd cortex compared to Pu.1+ nuclei from scramble cortex. Each row represents gene expression from 1245 one mouse.

Error bars represent standard error of mean (S.E.M.); **** $\mathrm{P}<0.0001, * * * \mathrm{P}<0.001, * * \mathrm{P}<0.01$, $* \mathrm{P}<0.05$; n.s. not significant. Student's t-test (h). One-way ANOVA followed by Holm-Sidak's test for multiple comparisons (i). h: Scramble $(n=5)$, p65kd $(n=5)$. i: CK $(n=7)$, Scramble $(n=5)$, p65kd $(n=6)$. 
Figure 5. Ccl2 and Cxcl10 are secreted from DSB-bearing neurons to activate microglia.

a. Schematic for treating acute $\mathrm{Cx} 3 \mathrm{cr} 1-\mathrm{GFP}$ slices with conditioned media from etoposide-treated primary neuron cultures. Cultures were either treated with 50uM ETP or vehicle control (DMSO) for six hours. In a separate condition, primary neurons were treated with 50uM ETP and 10uM NF-kappaB Activation Inhibitor VI (IKK2 inhibitor). Cultures were washed with PBS after six hours and media was replaced. After 24 hours, this media was applied to acute Cx3cr1-GFP slices for 6 hours.

b. Representative images of GFP in Cx3cr1-GFP acute slices treated with primary neuron conditioned media.

c-f. Quantification of branch length per microglia (c), end-points per microglia (d), soma area (e), and number of microglia per image (f). Each data point represents the average measurement from two images in one acute slice.

g,h. Quantification of $\mathrm{Cxcl10}(\mathrm{g})$ and $\mathrm{Ccl} 2(\mathrm{~h})$ from conditioned media from control and etoposidetreated primary neurons. Each datapoint represents one biological replicate.

i. Schematic of ETP conditioned media experiment. Primary neurons were treated with either ETP or DMSO for 6 hours, washed with PBS, then media was replaced. Cultures recovered for 24 hours before conditioned media was collected. IgG, Ccl2, or Cxcl10 antibodies were used to immunodeplete conditioned media before they were applied to Cx3cr1-GFP acute slices for 6 hours.

j. Representative images of microglia from acute slices treated with different conditioned media.

k-n. Quantification of (k) branch length per microgli (l) end-points per microglia, soma area (m), and number of microglia per image (n). Each data point represents the average measurement from two images in one acute slice.

Error bars represent standard error of mean (S.E.M.); ${ }^{* * * *} \mathrm{P}<0.0001,{ }^{* * *} \mathrm{P}<0.001,{ }^{*} \mathrm{P}<0.01$, $* \mathrm{P}<0.05$, ns not significant. Two-way ANOVA followed by Sidak's test for multiple comparisons (c-f). One-way ANOVA followed by Tukey's test for multiple comparisons (k-n). Data are combined from two independent experiments $(c-f, k-n)$. Data are combined from three independent experiments $(\mathrm{h}, \mathrm{i})$. 


\section{Supplementary Figure 1.}

1279 a. Representative images of $\gamma \mathrm{H} 2 \mathrm{AX}$ immunostaining in CK and CK-p25 cortex over a 6-week 1280 timeline analysis.

1281 b. Average number of $\gamma \mathrm{H} 2 \mathrm{AX}+$ nuclei quantified per image at each timepoint. Each data point 1282 represents one mouse.

1283 c. Flow cytometry dot plot of $\gamma \mathrm{H} 2 \mathrm{AX}+\mathrm{pATM}+$ nuclei in CK and CK-p25 cortex.

d. Quantificaton of $\gamma \mathrm{H} 2 \mathrm{AX}+\mathrm{pATM}+$ nuclei in $\mathrm{CK}$ and $\mathrm{CK}-\mathrm{p} 25$ cortex. Each data point represents percent $\gamma \mathrm{H} 2 \mathrm{AX}+\mathrm{pATM}+$ nuclei for one mouse.

e. Flow cytometry dot plot of $\gamma \mathrm{H} 2 \mathrm{AX}$, Camk2a, and NeuN immunoreactivity in nuclei from CKp25 cortex. $\gamma \mathrm{H} 2 \mathrm{AX}+\mathrm{Camk} 2 \mathrm{a}+$ nuclei are gated in the left graph. This gated population is highlighted in turquoise in the two graphs to the right.

f. Camk2a median fluorescent intensity (MFI) is quanitifed for Glia, Baseline, Stage 1, and Stage

2 populations. MFI values are normalized to the Glia population.

1291 g. Representative dot plot of $\gamma \mathrm{H} 2 \mathrm{AX}$ and NeuN immunoreactivity in 2-week induced CK-p25

1292 cortex.

1293 h. Representative images of cell type and $\gamma \mathrm{H} 2 \mathrm{AX}$ immunostaining in the 2wk-induced CK-p25 cortex. Celltype markers from left to right: neurons (NeuN), astrocytes (GFAP),

1295 oligodendrocytes and oligodendrocyte precursor cells (Olig2), microglia (Iba1).

1296 i. Quantification of percent of $\gamma \mathrm{H} 2 \mathrm{AX}$-positive nuclei overlapping with each celltype stain. Each dot represents one mouse. 50-100 $\gamma \mathrm{H} 2 \mathrm{AX}$-positive nuclei were analyzed for each mouse and 1298 celltype marker.

1299 j. Expression p25 in CK and CK-p25 gated populations. One datapoint represents fragments per kilobase per million (FPKM) from a gated population from one mouse.

1301 k. Representative images of GFP, $\gamma \mathrm{H} 2 \mathrm{AX}$, and NeuN immunostaining from CK and CK-p25 cortex at the $2 \mathrm{wk}$ time point. Arrowheads indicate $\gamma \mathrm{H} 2 \mathrm{AX}+$ nuclei that express $\mathrm{p} 25-\mathrm{GFP}$ regardless of NeuN immunoreactivity.

1304 I. Quantification of GFP expression across Stage 1 and Stage 2 populations. Each data point 1305 represents percent $\gamma \mathrm{H} 2 \mathrm{AX}+$ population from Stage 1 or Stage 2 from one CK-p25 mouse.

1306 m. Representative images of Neurod1, $\gamma \mathrm{H} 2 \mathrm{AX}$, and NeuN from CK-p25 cortex at two-week 1307 timepoint. White arrowhead indicates a $\gamma \mathrm{H} 2 \mathrm{AX}+$ nucleus with Neurod 1 immunoreactivity but not NeuN immunoreactivity. 
1309 n. Quantification of Neurod1 expression across Stage 1 and Stage 2 populations. Each data point 1310 represents percent $\gamma \mathrm{H} 2 \mathrm{AX}+$ population from Stage 1 or Stage 2 from one CK-p25 mouse.

1311 Error bars represent standard error of mean (S.E.M.); $* * * * \mathrm{P}<0.0001, * * * \mathrm{P}<0.001,{ }^{*} \mathrm{P}<0.05$, n.s. 1312 not significant; One-way ANOVA with Tukey's test for multiple comparisons (b,f). Student's T1313 test (d). Data are averages of 4 images per mouse (b). Data are representative of at least 2 1314 independent experiments (d). Data are pooled from 2 independent experiments (f). 


\section{Supplementary Figure 2.}

a. Principle component analysis (PCA) of normalized gene expression matrix from $\mathrm{CK}$ and $\mathrm{CK}$ p25 subpopulations. Principle component 1 (PC1): 66\% variance. Principle component 2 (PC2): $29 \%$ variance.

b. Volcano plots of (top) Stage 1 vs. CK-p25 Baseline and (bottom) Stage 2 vs. CK-p25 Baseline contrasts. Gray circles: non-significant (ns) transcripts. Red circles: transcripts with FDR adjusted p-value $<0.05$ and $\log 2$ fold change $>|0.1|$.

c. Heatmap of differentially expressed genes from Stage 2 vs. CK-p25 Baseline and Stage 1 vs. CK-p25 Baseline contrasts. Each column represents one mouse.

d. Representative image of RNAscope probes for Camk2a (yellow) and Cxcl10 (magenta), and $\gamma \mathrm{H} 2 \mathrm{AX}$ immunostaining (turquoise). To the right, the percent of $\gamma \mathrm{H} 2 \mathrm{AX}$-positive cells that are Camk2a-positive ( 2 or more Camk2a puncta within the nucleus) are quantified. Each datapoint represents one 2-week induced CK-p25 mouse. 17-42 $\gamma \mathrm{H} 2 \mathrm{AX}$-positive cells were quantified for each mouse. Turquoise outlines indicate $\gamma \mathrm{H} 2 \mathrm{AX}$-positive Camk2a-positive cells. Gray outlines indicate $\gamma \mathrm{H} 2 \mathrm{AX}$-negative cells. White arrows indicate Camk2a-negative cells.

e. Representative image of the RNAscope probe for Cxcl10 (magenta) combined with $\gamma \mathrm{H} 2 \mathrm{AX}$ immunostaining (turquoise). Imaging was performed on $1 \mathrm{wk}$ and $2 \mathrm{wk}$ CK-p25 cortices. To the right, the number of $\gamma \mathrm{H} 2 \mathrm{AX}$-positive and $\gamma \mathrm{H} 2 \mathrm{AX}$-negative cells with 2 or more Cxc110 puncta are quantified. Each datapoint represents the average \%Cxcl10-positive cells in one image from one mouse. 4-3 images were taken per mouse. (CK-p25 1wk n=4, CK-p25 2wk n=4).

f. Representative dot plots of $\gamma \mathrm{H} 2 \mathrm{AX}$ and NeuN immunoreactivity in CK and CK-p25 mice at 1, 2 , and 6 weeks induction. Stage 1 and Stage 2 percent population was calculated with respect to the total $\gamma \mathrm{H} 2 \mathrm{AX}$-positive population.

1338 g. Stage 1 and Stage 2 percent population are quantified for each timepoint. $* \mathrm{P}<0.05$, n.s. not significant; Two-way ANOVA followed by Sidak's test for multiple comparisons $(e, g)$. Data are representative of 2 independent experiments (d). Data are pooled from four independent experiments $(\mathrm{g})$. 


\section{Supplementary Figure 3.}

1344 a. UMAPs labeled by genotype, mouse id, and timepoint.

1345 b. Distribution of Stage 2-gated nuclei in in silico cell type clusters, stratified by biological 1346 replicate.

1347 c. Percent FANS label distribution across scRNA cell type clusters. Bargraph colors refer to FANS 1348 gate label.

1349 d. Bulk RNA-seq gene signature enrichment in neuron cell type clusters. Stage 1 signature 1350 enrichment (left), Stage 2 signature enrichment (middle), and immune genes from Stage 2 1351 enrichment (right). Each datapoint indicates average gene expression across all cells from one 1352 mouse. Only CK-p25 mice were used for this analysis.

$1353 * * * * \mathrm{P}<0.0001, * * * \mathrm{P}<0.001, * * \mathrm{P}<0.01, * \mathrm{P}<0.05$, n.s. not significant; One-way ANOVA with 1354 Tukey's test for multiple comparisons (d). 


\section{Supplementary Figure 4.}

1356 a. Representative images of celltype immunostaining in DIV13 primary neuron cultlures. The percent nuclei that stained positively for each celltype marker are quantified on the right. Three images were analyzed for each marker.

1359 b. PCA plot of normalized gene expression matrix from ETP and DMSO-treated neurons. Principle component 1 (PC1): 75\% variance. Principle component 2 (PC2): 7\% variance.

1361 c. Volcano plot of ETP vs. DMSO contrast. Gray circles: non-significant (ns) transcripts. Red 1362 circles: transcripts with FDR adjusted p-value $<0.05$ and $\log 2$ fold change $>|0.1|$.

1363 d. Heatmap of differentially expressed genes from ETP vs. DMSO contrast. Columns represent 1364 biological replicates.

1365 e. Quantification of Stage 1 and Stage 2 signature enrichment (from bulk RNAseq) in DMSO and 1366 ETP-treated neurons. Each data point represents one biological replicate.

1367 f. Number of $\mathrm{Ccl} 2$ puncta (magenta) are quantified for Rbfox3-positive nuclei (2 or more Rbfox3 1368 puncta within or surrounding the nucleus, yellow), and Rbfox3-negative nuclei. Cyan circles 1369 outline nuclei. Each datapoint represents one nucleus.

1370 Error bars represent standard error of mean (S.E.M.); ****P<0.0001, *** $\mathrm{P}<0.001, * \mathrm{P}<0.05$. 1371 Wilcoxon test (e). Student's t-test (f). Data are representative of two independent experiments $(\mathrm{a}, \mathrm{f})$. 


\section{Supplementary Figure 5.}

1373 a. Flow cytometry dot plot of $\gamma \mathrm{H} 2 \mathrm{AX}$ gating for human $\mathrm{NeuN}+$ and NeuN- nuclei. Percent total 1374 population is indicated for each gating box.

1375 b. Correlation heatmap of $\gamma \mathrm{H} 2 \mathrm{AX}+$ and $\gamma \mathrm{H} 2 \mathrm{AX}-/ \mathrm{NeuN}+$ and NeuN- bulk RNAseq libraries from 1376 postmortem temporal cortex.

1377 c. $\mathrm{PCA}$ plot of normalized gene expression from $\gamma \mathrm{H} 2 \mathrm{AX}+$ and $\gamma \mathrm{H} 2 \mathrm{AX}-/ \mathrm{NeuN}+$ and $\mathrm{NeuN}-$ 1378 samples.

1379 d. Quantification of normalized neuronal and glial marker gene expression in NeuN+ and NeuNsamples. Mean gene expression is compared with a Wilcoxon test. Gene set enrichment was calculated using Gene Set Variation Analysis (GSVA).

1382 e. Gene expression heatmap of top five marker genes for major brain cell types. Each column 1383 represents one human sample.

1384 f. Gene expression heatmap of all marker genes for major brain cell types. Each column represents 1385 one human sample.

1386 g. Distribution of $\operatorname{logFC}$ values for Stage 1 genes (light blue), Stage 2 genes (dark blue), and other genes (red). $\operatorname{LogFC}$ values are from $\gamma \mathrm{H} 2 \mathrm{AX}+\mathrm{vs} \gamma \mathrm{H} 2 \mathrm{AX}$ - comparison. Mean LogFC for each gene set are compared with a Wilcoxon test.

h. Heatmap of ETP and $\gamma \mathrm{H} 2 \mathrm{AX}+$ and $\gamma \mathrm{H} 2 \mathrm{AX}$ - human neuronal nuclei transcriptional correlation.

1390 Excitatory (Ex), Inihibtory (In), Astrocyte (Ast), Microglia (Mic), Oligodendrocyte (Oli), 


\section{Supplementary Figure 6.}

1393 a. Schematic for binning $\gamma \mathrm{H} 2 \mathrm{AX}$ expression in NeuN-positive cells, and nuclear p65 1394 quanitification. For each individual, the median $\gamma \mathrm{H} 2 \mathrm{AX}$ expression in NeuN-positive cells was 1395 calculated. Cells were then binned as $\gamma \mathrm{H} 2 \mathrm{AX}$ high or $\gamma \mathrm{H} 2 \mathrm{AX}$ low based off of median $\gamma \mathrm{H} 2 \mathrm{AX}$ 1396 expression. Nuclear p65 intensity was then calculated for all cells.

1397 b. Raw data from the four AD individuals used for analysis.

1398 Error bars represent standard error of mean (S.E.M.); **** $\mathrm{P}<0.0001, * * * \mathrm{P}<0.001, * * \mathrm{P}<0.01$, $1399 * \mathrm{P}<0.05$, ns not significant. A t-test was performed to compare mean p65 intensity between $1400 \gamma \mathrm{H} 2 \mathrm{AX}$ low and $\gamma \mathrm{H} 2 \mathrm{AX}$ high cells. 


\section{Supplementary Figure 7.}

1402 a. Distrubution of capture areas amongst spatial clusters for each sample. The y-axis refers to 1403 number capture areas. Colors refer to spatial cluster id.

1404 b. Distribution of samples by spatial cluster. Colors refer to sample id.

1405 c. Top: $\gamma \mathrm{H} 2 \mathrm{AX}$ immunostaining for each CK-p25 section. Bottom: the corresponding capture 1406 areas identified as $\gamma \mathrm{H} 2 \mathrm{AX}$-positive are marked in yellow. $\gamma \mathrm{H} 2 \mathrm{AX}$-negative capture areas are 1407 marked in purple. 


\section{Supplementary Figure 8.}

1409 a. Gene set enrichment analysis of $\gamma \mathrm{H} 2 \mathrm{AX}$-positive capture area DEGs. DEGs were tested for 1410 the enrichment of the reactive microglia signature characterized in Mathys et al., 2019. The 1411 analysis was first performed for all $\gamma \mathrm{H} 2 \mathrm{AX}$-posistive capture areas, then for individual clusters 1412 comprised of $20 \%$ or more $\gamma \mathrm{H} 2 \mathrm{AX}$-positive capture areas. Normalized enrichment score (NES).

1413 b. Percent $\gamma \mathrm{H} 2 \mathrm{AX}$-positive capture areas by spatial cluster. Orange indicates $\gamma \mathrm{H} 2 \mathrm{AX}$-postive 1414 capture areas. Blue indicates $\gamma \mathrm{H} 2 \mathrm{AX}$-negative capture areas. 


\section{Supplementary Figure 9.}

1416 a. Distribution of spatial clusters for each sample.

1417 b-d. Expression of reactive microglia and DSB-bearing neuron genes (b) Ifitm3, (c) H2-D1, and 1418 (c) Cxcl10 for each sample.

1419 e. Distribution of $\gamma \mathrm{H} 2 \mathrm{AX}$-positive capture areas for each CK-p25 sample. 


\section{Supplementary Figure 10.}

1421 a. Representative images of cell type specific immunostaining and RFP immunostaining in 1422 scramble and p65kd cortex. Top to bottom, left to right: NeuN=neurons, Iba1=microglia, $1423 \gamma \mathrm{H} 2 \mathrm{AX}=\gamma \mathrm{H} 2 \mathrm{AX}+$ cells, Olig2=oligodendrocytes and oligodendrocyte precursor cells, 1424 GFAP=astrocytes.

1425 b. Quantification of cell type distribution of RFP+ cells. Total number of RFP+ cells are quantified 1426 per image, then the fraction co-stained for a celltype marker are calculated. Each datapoint 1427 represents one mouse.

1428 c. P65 and RFP immunostaining in scramble and p65kd CK-p25 cortex.

1429 d. Quantification of p65 mean intensity. Analysis was performed on three animals, two sections 1430 each. Scramble $n=113$ cells, $\mathrm{p} 65 \mathrm{kd} n=103$ cells.

1431 e. qRT-PCR of p65 in RFP+ NeuN+ nuclei from scramble and shp65-treated CK-p25 mice.

1432 f. Representative images of $\gamma \mathrm{H} 2 \mathrm{AX}$ immunostaining in p65 kd and scramble CK-p25 cortex.

1433 g. Number of $\gamma \mathrm{H} 2 \mathrm{AX}+$ per image are quantified. Each data point represents one mouse.

1434 Error bars represent standard error of mean (S.E.M.); **** $\mathrm{P}<0.0001, * * * \mathrm{P}<0.001, * * \mathrm{P}<0.01$, $1435 * \mathrm{P}<0.05$, n.s. not significant. Student's t-test $(\mathrm{d}, \mathrm{e}, \mathrm{g})$. 


\section{Supplementary Figure 11.}

1437 a. Sorting schematic for RFP $+\gamma \mathrm{H} 2 \mathrm{AX}+$ and $\gamma \mathrm{H} 2 \mathrm{AX}$ - neurons. 30,000 nuclei were collected for 1438 each gate for each animal.

1439 b. Representative images of Ccl2 RNAscope combined with $\gamma \mathrm{H} 2 \mathrm{AX}$ immunofluorescence in 1440 Scramble and p65kd cortex.

1441 c. Quantification of Ccl2 puncta per $\gamma \mathrm{H} 2 \mathrm{AX}+$ cell. Each datapoint represents one cell ( $\mathrm{n}=197 \mathrm{for}$ 1442 scramble, $\mathrm{n}=157$ for $\mathrm{p} 65 \mathrm{kd}$ ). 20-40 cells were analyzed per mouse.

1443 d. Sorting schematic for Pu.1+ nuclei for RNA-sequencing.

1444 e. Quantification of total percent Pu.1+. One datapoint represents one mouse.

1445 f. PCA plot of normalized gene expression matrix from Pu.1+ bulk RNA-sequencing.

1446 g. Volcano plot from P65 vs. Scramble contrast.

1447 Error bars represent standard error of mean (S.E.M.); **** $\mathrm{P}<0.0001, * * * \mathrm{P}<0.001, * * \mathrm{P}<0.01$, $1448 * \mathrm{P}<0.05$, ns not significant. One-way ANOVA followed by Tukey's test for multiple comparisons 1449 (e). 


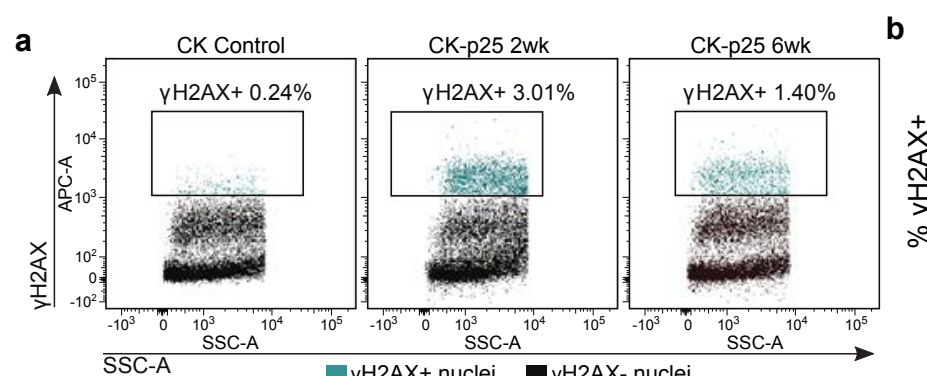

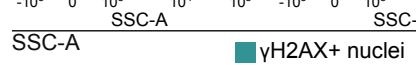

bioRxiv preprint doi: https://doi.org/10.1101/2021.12.23.474

Week

c

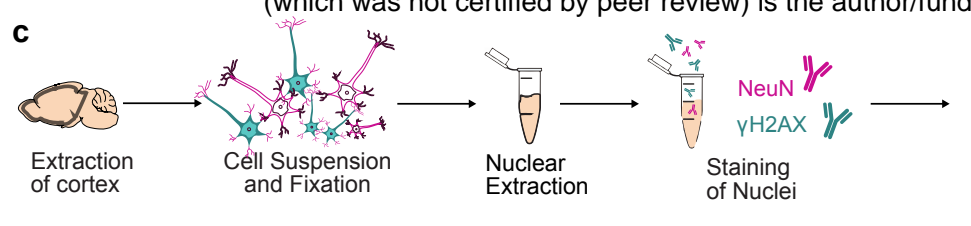

b 13$\urcorner r^{* * * *} \longmapsto$

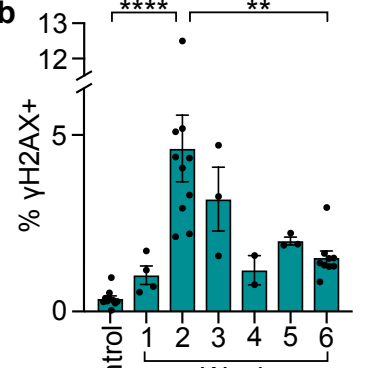

d

NES

$\geq 0.25$

Mitotic nuclear division

Signal transduction by P53 class mediator Chromatin assembly
Cell cycle checkpoint Intrinsic apoptotic sign. path. in resp. to DNA damage DNA double-strand break repair Senescence-associated secretory phenotype (SASP)

Cytosolic sensors of pathogen-associated DNA Hallmark apoptosis .

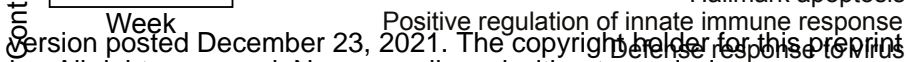

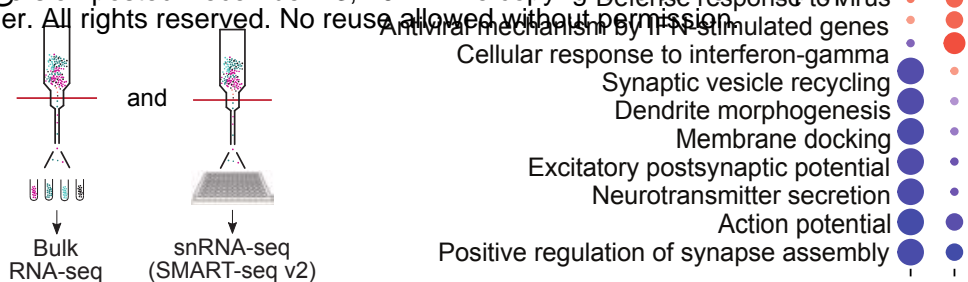

STAGE: 12
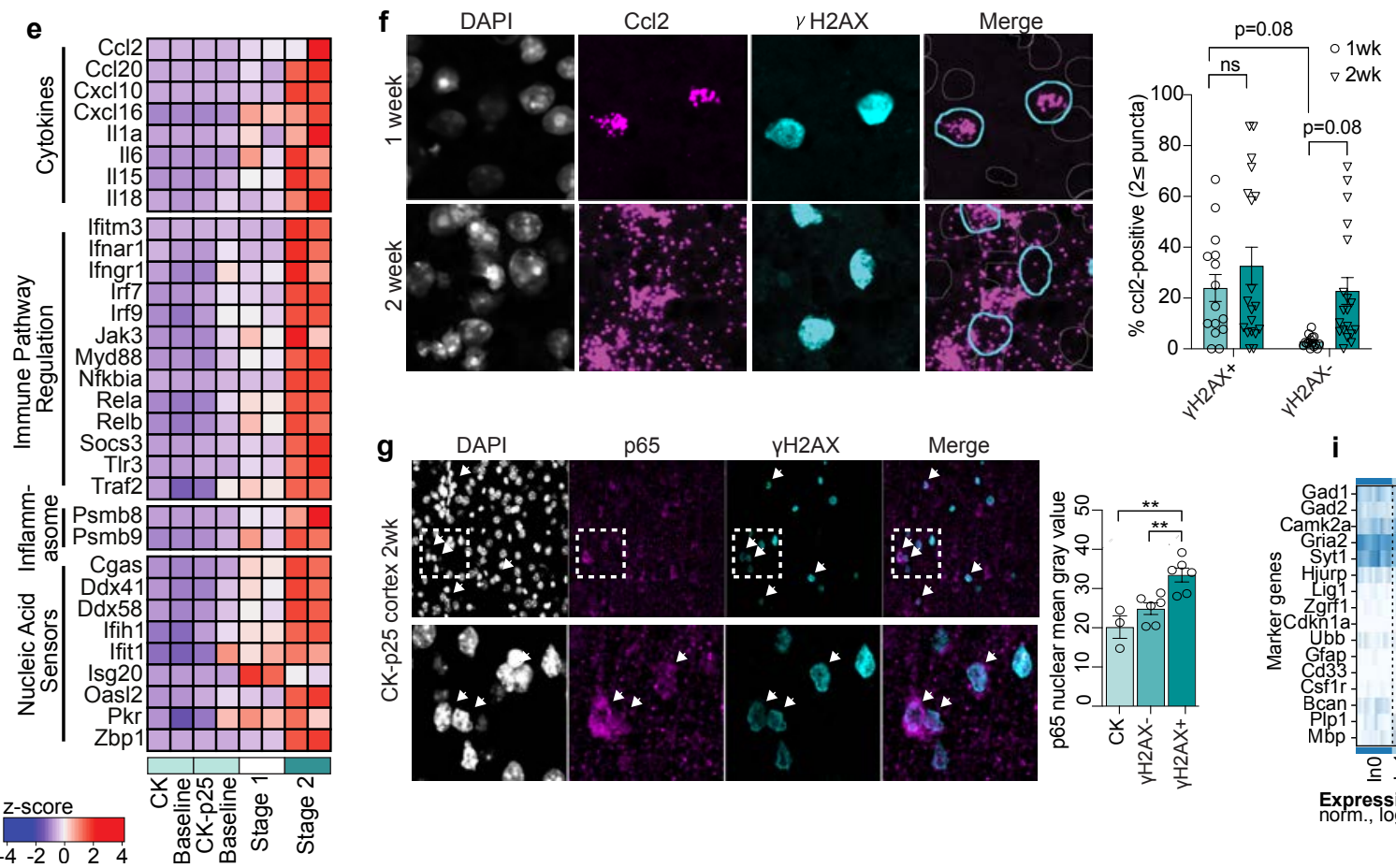

h

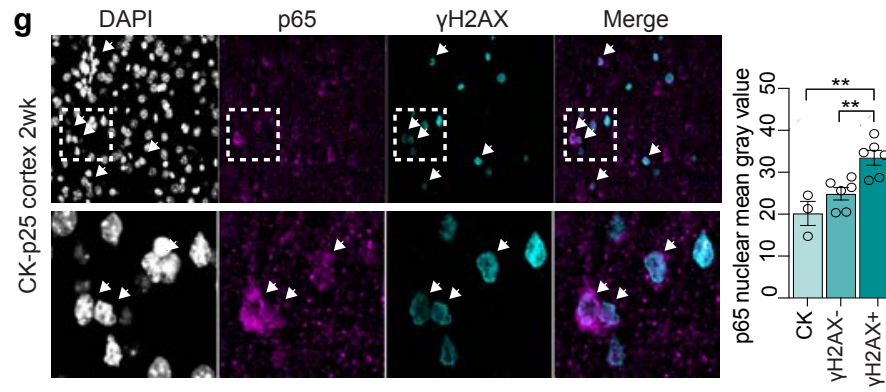

$x^{2 v^{+}}$

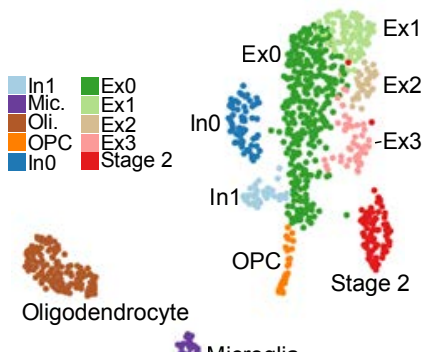

Microglia
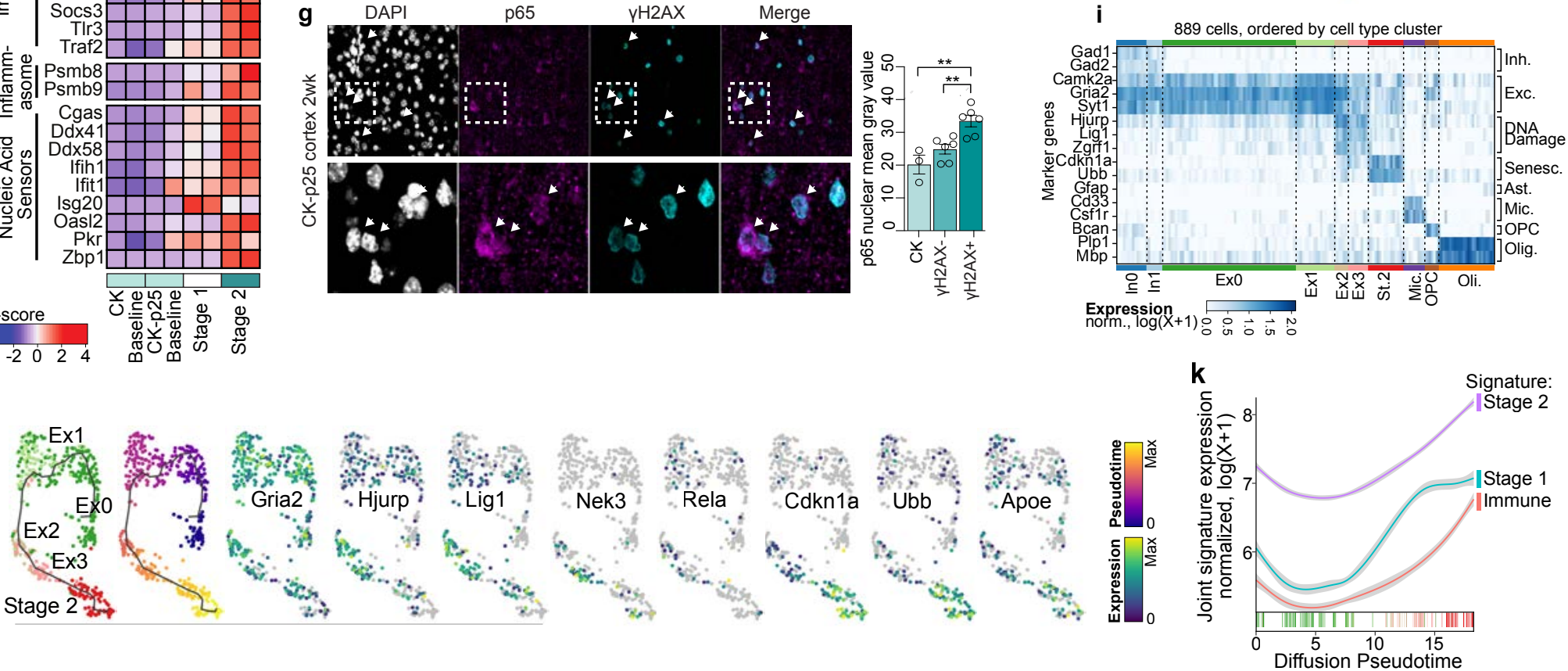

Figure 1. Neurons marked by DNA DSBs activate inflammatory signaling at early stages of neurodegeneration.

a. Flow cytometry dot plots of $\mathrm{yH} 2 \mathrm{AX}+$ nuclei from $\mathrm{CK}$ and $\mathrm{CK}-\mathrm{p} 25$ cortex. $\mathrm{yH} 2 \mathrm{AX}+$ are highlighted in turquoise, and percent total population is indicated above the gating box.

b. CK-p25 mice were taken off dox for 1 through 6 weeks, then cortical nuclei were extracted and stained for $\mathrm{yH} 2 \mathrm{AX}$. Each datapoint represents percent $\mathrm{yH} 2 \mathrm{AX}+$ nuclei from one mouse cortex.

c. RNA seq workflow for the gated populations. Bulk RNA-seq: Cortices were extracted from CK and CK-p25 mice (n=2 per genotype for bulk RNA-seq, 2 -week timepoint only). Tissue was homogenized, then nuclei were extracted and stained for NeuN and $\mathrm{YH} 2 \mathrm{AX}$. 50,000 nuclei were sorted from each gated population. snRNA-seq: Cortices were extracted from CK and CK-p25 mice ( $n=3$ per genotypextimepoint for SMART-seq, 1-week and 2-week timepoints). Tissue was homogenized, then nuclei were extracted and stained for NeuN and yH2AX. 48 nuclei were sorted for each gated CK-p25 population per mouse, and 32 nuclei were sorted for each gated CK population per mouse.

d. Differential gene ontology terms from gene set enrichment analysis (GSEA) of bulk RNAseq data. Left column depicts GSEA results from Stage 1 vs. CK-p25 Baseline contrast. Right column depicts GSEA results from Stage 2 vs. CK-p25 Baseline contrast. Color indicates normalized enrichment score (NES). Size indicates false discovery rate (FDR).

e. Heatmap of differentially expressed genes belonging to inflammatory gene sets from bulk RNA-seq data. Each column represents one mouse. Gene sets are organized by biological function.

f. (left): Representative images of $\mathrm{Ccl} 2 \mathrm{RNAscope} \mathrm{combined} \mathrm{with} \mathrm{yH} 2 \mathrm{AX}$ immunofluorescent staining. Imaging was performed on 1 wk and 2 wk CK-p25 cortex (right): Quantification of number of $\mathrm{yH} 2 \mathrm{AX}+$ and $\mathrm{yH} 2 \mathrm{AX}$ - cells with $2 \leq \mathrm{Ccl} 2$ puncta. Each datapoint respresents average \%Ccl2-positive cells in one image from one mouse. 4-3 images were taken per mouse. (Ccl2 1 wk n=4, Ccl2 2wk n=5)

g. Representative image of p65 immunostaining in CK-p25 cortex at two-week timepoint. White arrowheads indicate $\mathrm{yH} 2 \mathrm{AX}+$ nuclei with nuclear p65 immunoreactivity. Quantification of p65 mean intensity for $\mathrm{yH} 2 \mathrm{AX}+$ and $\mathrm{yH} 2 \mathrm{AX}$ - nuclei. Each data point represents the average p65 nuclear mean gray value of 20-60 nuclei from one mouse.

h. UMAP of gated populations from CK and CK-p25 cortex at 1-week and 2-week timepoints. Colors indicate cell type annotation.

i. Marker gene expression for each cell type cluster. Columns represent 985 individual cells ordered by cell type cluster.

j. Trajectory analysis of Ex0,1,2,3, and Stage 2 neurons. Cells from each cluster (indicated by color annotation) are ordered across pseudotime (indicated by color gradient).

k.Trajectory analysis of Ex0,1,2,3, and Stage 2 neurons. Smoothened gene signature expression across pseudotime. Stage $1=$ Significantly upregulated genes from Stage 1 vs. Baseline contrast. Stage 2= Significantly upregulated genes from Stage 2 vs. Baseline contrast. Immune= Genes belonging to immune gene ontologies that were significantly upregulated in Stage 2 neurons. padj $<0.05, \log _{2}$ fold-change $\geq 1.0$.

Error bars represent standard error of mean (S.E.M.); ${ }^{* * *} \mathrm{P}<0.0001,{ }^{* *} \mathrm{P}<0.001,{ }^{* *} \mathrm{P}<0.01,{ }^{*} \mathrm{P}<0.05$; One-way ANOVA with Tukey's test for multiple comparisons $(\mathrm{b}, \mathrm{f})$. Two-way ANOVA followed by Sidak's test for multiple comparisons $(\mathrm{g})$. Data are pooled from 4 independent experiments (b). Data are representative of two independent experiments $(\mathrm{f}, \mathrm{g})$. 

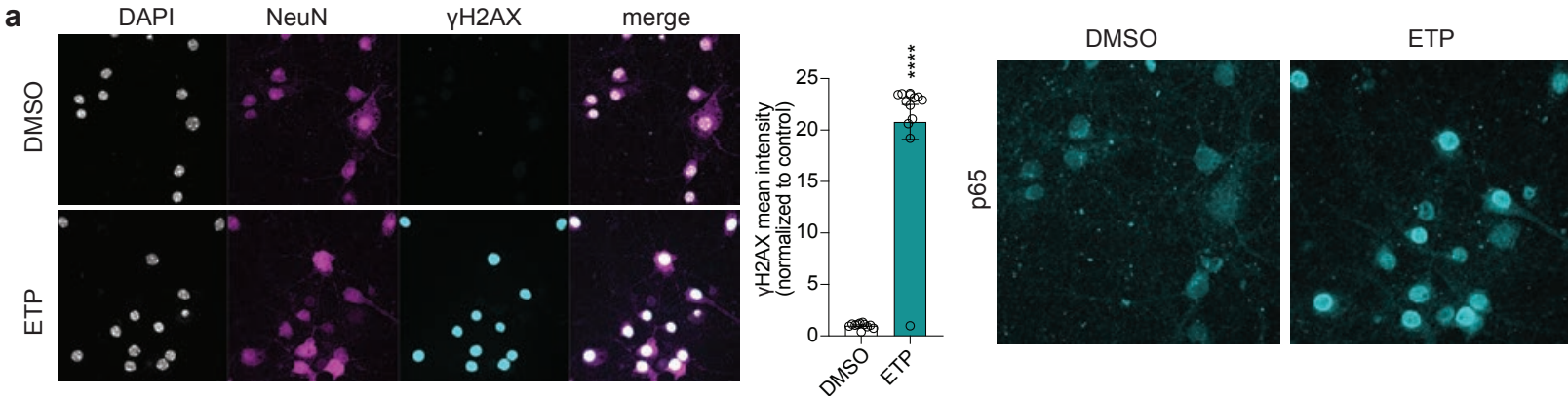

DIV 13 mouse primary neurons

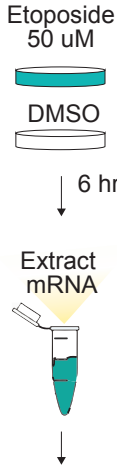

RNA-seq
C

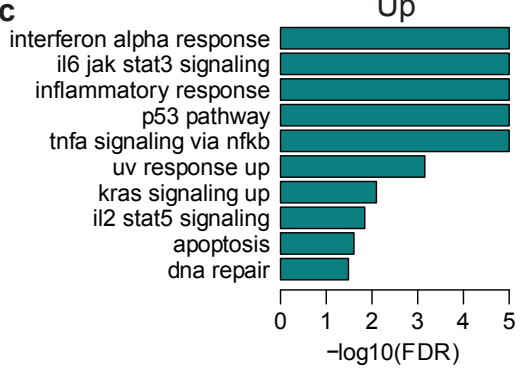

Down

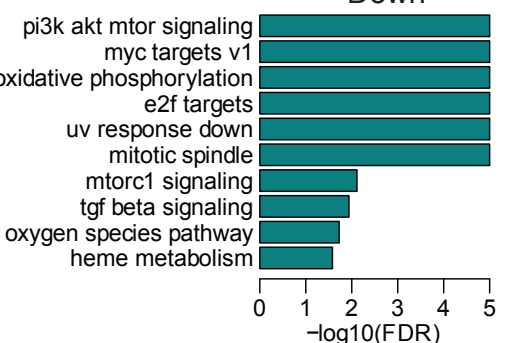

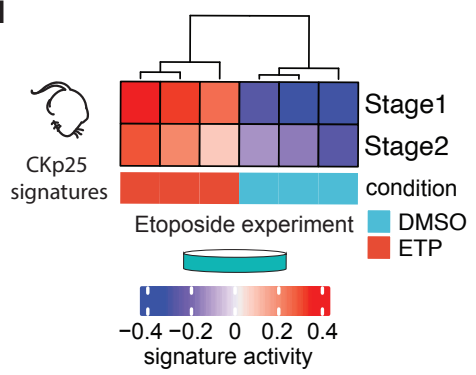

e

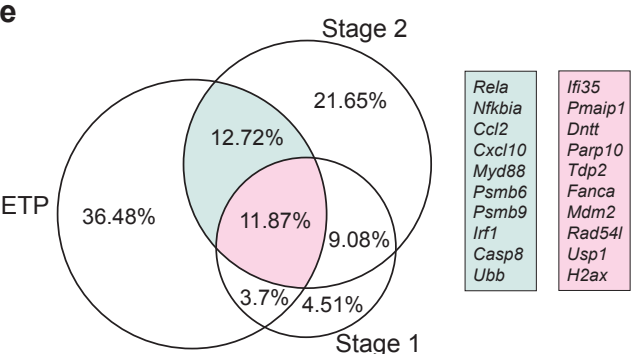

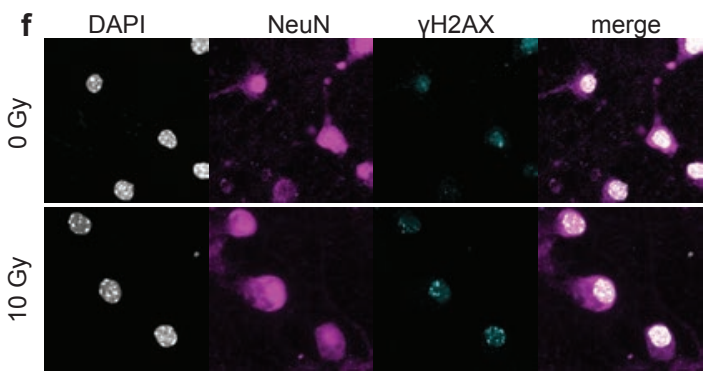
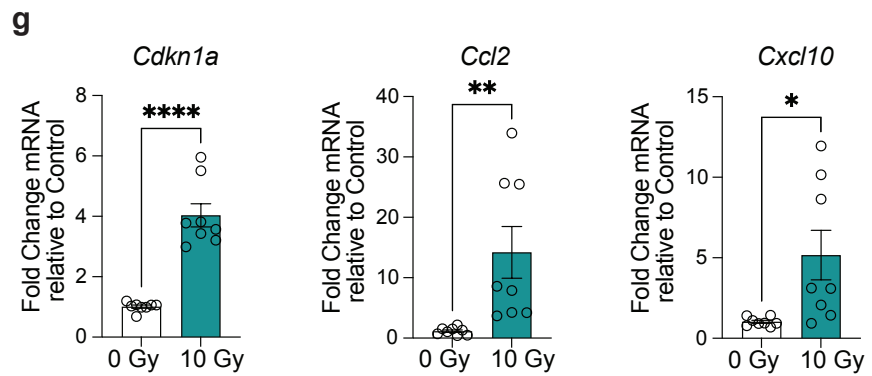

Figure 2. Induction of DNA DSBs is sufficient to elicit immune pathway signaling in neuron primary culture.

a. Top: Representative images of $\mathrm{NeuN}$ and $\mathrm{yH} 2 \mathrm{AX}$ immunostaining in ETP and vehicle-treated primary cultures. $\mathrm{yH} 2 \mathrm{AX}$ immunoreactivity is quantified for each condition. Each data point represents $\mathrm{yH} 2 \mathrm{AX}$ mean gray value for one nucleus in the representative image. Bottom: Representative images of p65 immunostaining in ETP and vehicle-treated primary cultures. p65 immunoreactivity is quantified for each condition. Each data point represents p65 mean gray value for one nucleus in the representative image.

b. Schematic of etoposide (ETP) treatment. DIV13 neuron primary culture were treated with either 50uM ETP or vehicle control (DMSO) for 6 hours. mRNA was extracted from the cultures and sequenced.

c. Differential gene ontology terms identified through gene set enrichment analysis (GSEA) from ETP vs. DMSO contrast.

d. Heatmap of Stage 1 and Stage 2 signature enrichment (from bulk RNAseq) in DMSO and ETP-treated neurons.

e. Venn diagram of significantly upregulated protein-coding genes from ETP-treated neurons and Stage 1 and Stage 2 gene signatures. Percentages are in reference to the total number of unique genes from all three gene sets. Example genes are shown to the right. Genes overlapping in ETP and Stage 2 are in turquoise. Genes overlapping in ETP, Stage 2, and Stage 1 are in magenta. The area of the circles are in proportion to the size of the gene sets.

f. Representative images of NeuN and $\mathrm{yH} 2 \mathrm{AX}$ immunostaining from $10 \mathrm{~Gy}$ and $0 \mathrm{~Gy} \mathrm{x}$-ray irradiation treated primary neurons.

g. qRT-PCR of Cdkn1a, Ccl2, and Cxcl10 in $10 \mathrm{~Gy}$ irradiated primary neurons. Each datapoint represents one biological replicate.

Error bars represent standard error of mean (S.E.M.); ${ }^{* * *} \mathrm{P}<0.0001,{ }^{* * *} \mathrm{P}<0.001$. Student's T-test (a,g). Data are representative of 3 independent experiments (a). Data are representative of 2 independent experiments (g). 
b

$A D$ cortex single-nucleous transcriptomes
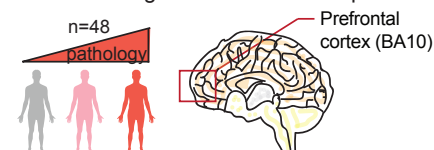

Mathys et al. 2019
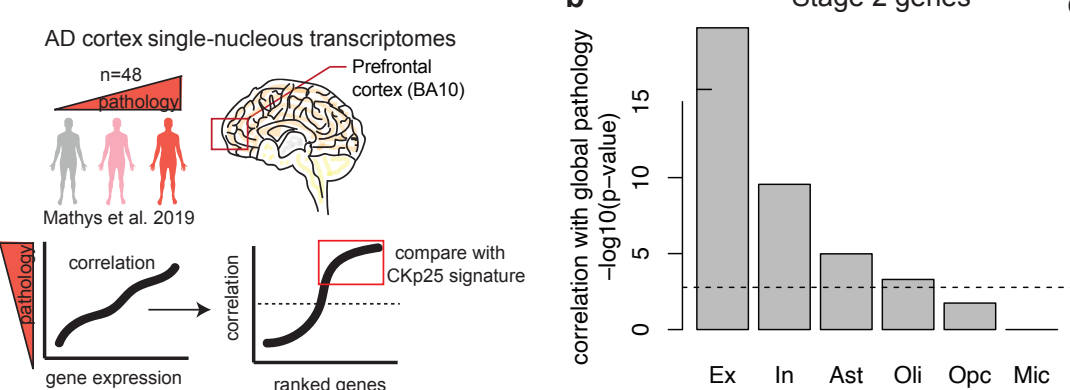

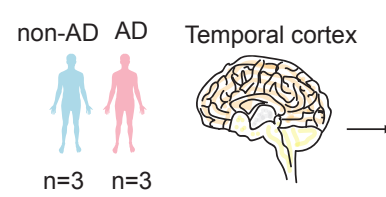

Nuclei Extraction

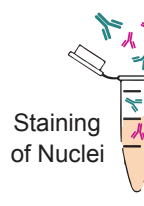

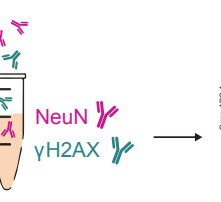

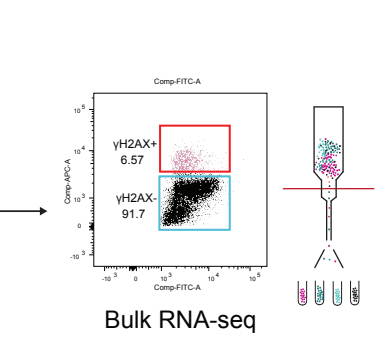

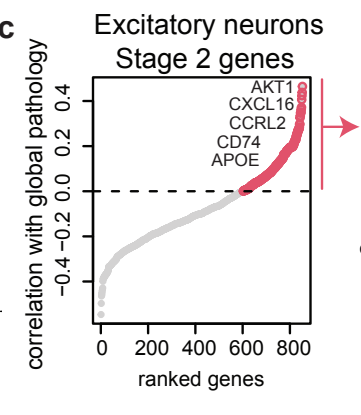

d
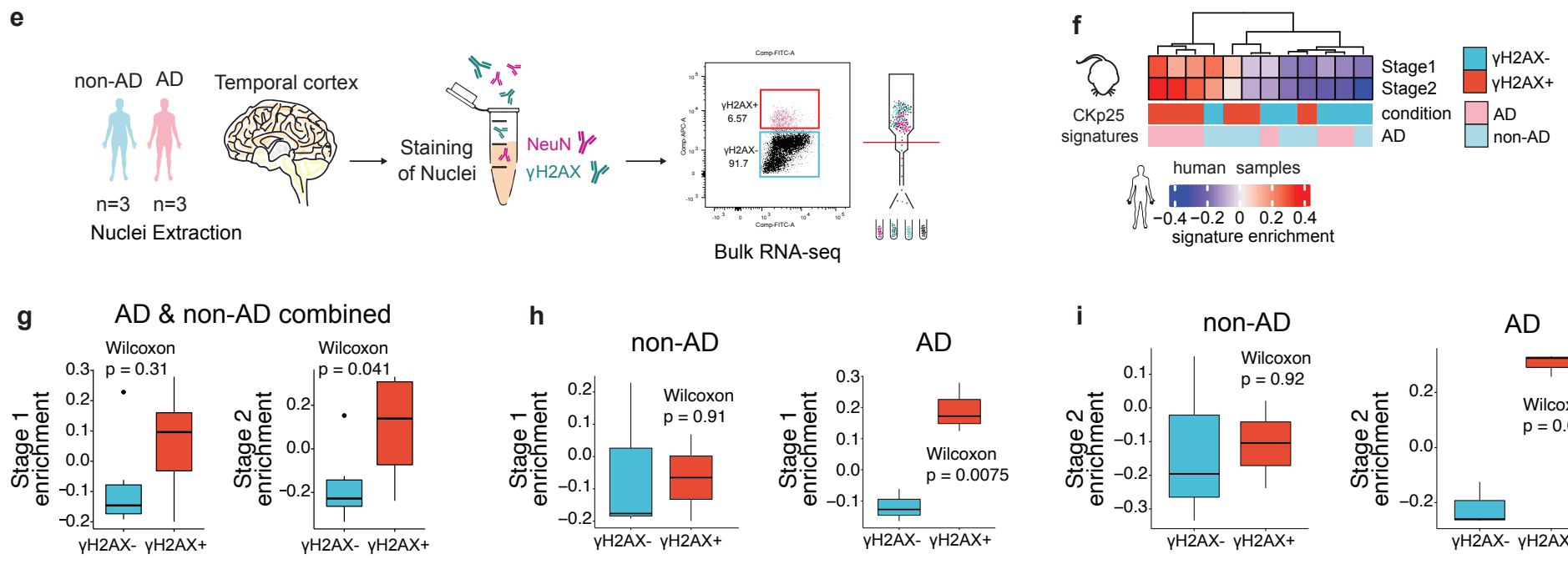

h
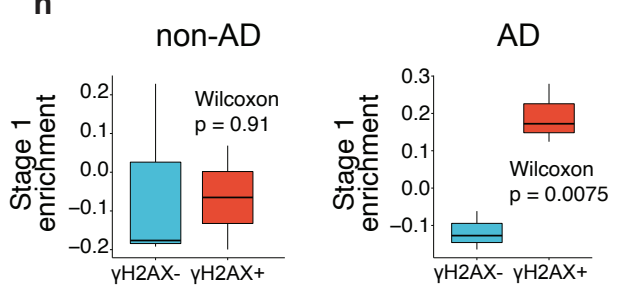

i

non-AD
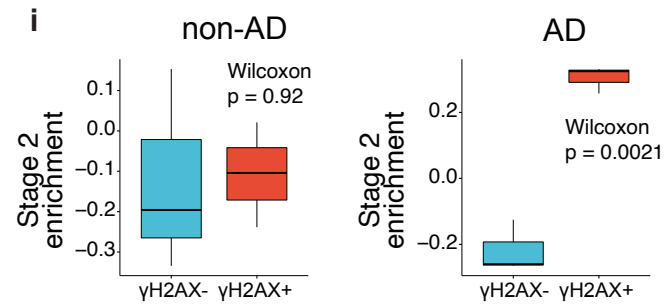

j
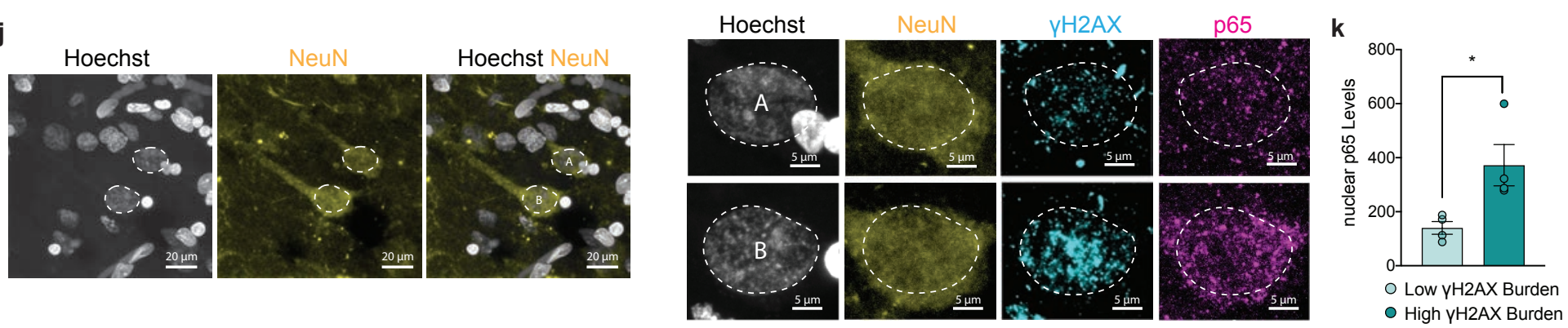

Figure 3. Inflammatory signaling in DSB-bearing neurons is positively correlated with Alzheimer's disease pathology.

a. Schematic of the Stage 2 signature analysis in the snRNA-seq dataset from Mathys et al., 2019.

b. Quantification of Stage 2 signature correlation with global pathology in celltype clusters from Mathys et al., 2019. It was tested if stage 2 genes were significantly and positively correlated with the global pathology metric for each major celltype. The $-\log _{10} p$-value for these tests are shown in the histogram. The dashed line indicates a p-value of 0.01 after Bonferroni correction for multiple testing. Excitatory neurons (Ex), Inhibitory neurons (In), Astrocytes (Ast), Oligodendrocytes (Oli), Oligodendrocyte precursor cells (Opc), Microglia (Mic).

c. Stage 2 signature genes ranked by their correlation with global pathology in excitatory neurons. Stage 2 genes with positive correlation are shown with red circles.

d. Gene ontology enrichment of Stage 2 signature genes positively correlated with global pathology.

e. Schematic of $\mathrm{YH} 2 \mathrm{AX}+$ nuclei sorting from $\mathrm{AD}$ and non-AD brain tissue.

f. Heatmap of Stage 1 and Stage 2 signature enrichment in $\mathrm{\gamma H} 2 \mathrm{AX}+$ and $\mathrm{\gamma H} 2 \mathrm{AX}$ - human NeuN+ nuclei.

g-i. Quantification of Stage 1 and Stage 2 signature enrichment in $\mathrm{yH} 2 \mathrm{AX}+$ and $\mathrm{yH} 2 \mathrm{AX}-$ human NeuN+ nuclei samples by FANS gate (g), and FANS gate and disease status $(\mathrm{h}, \mathrm{i})$.

j. Representative image of $\mathrm{yH} 2 \mathrm{AX}$, p65, and NeuN in the AD brain. Left: two NeuN-positive nuclei are outlined (white dashed line). Right: magnification of the two outlined nuclei. Top nucleus $(A)$ represents low $\mathrm{YH} 2 \mathrm{AX}$ burden, bottom nucleus $(B)$ represents high $\mathrm{YH} 2 \mathrm{AX}$ burden.

k. Quantification of p65 nuclear enrichment in low and high y $\mathrm{H} 2 \mathrm{AX}$-burdened neurons. Each dot represents the average of $23-41 \mathrm{NeuN}+$ nuclei per individual. 
a

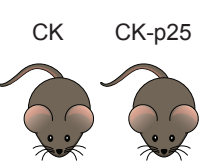

$\downarrow$

Induce p25

Exprie日3ixiv preprint doi: https://doi.org//\%0.1101/2021.

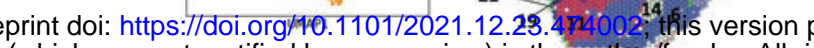

b

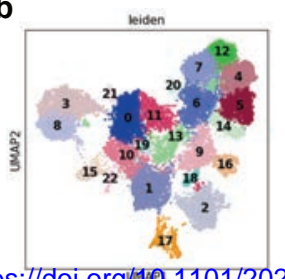

C

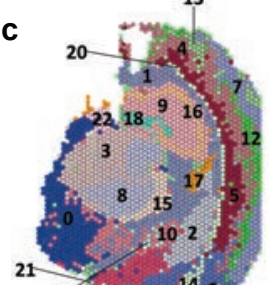

f

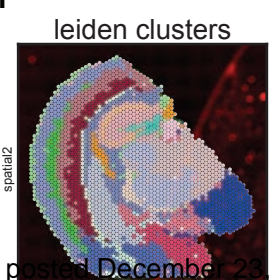

yH2AX-positive

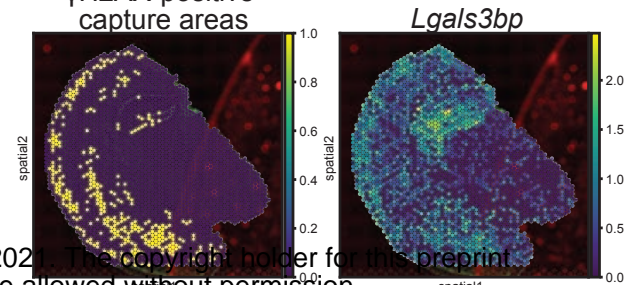

$H 2-D 1$
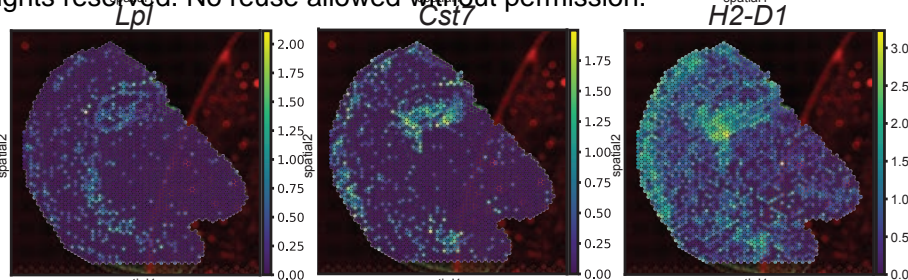

g

h
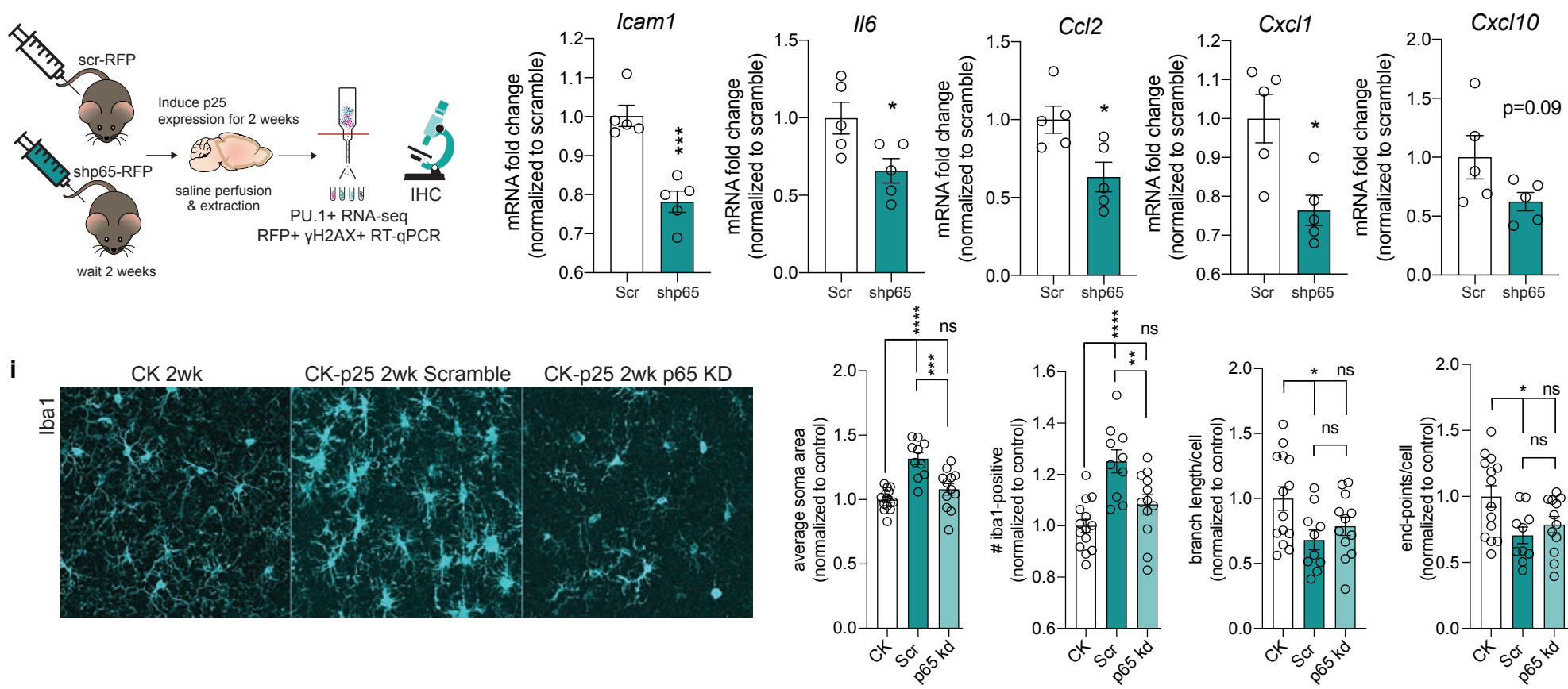

j

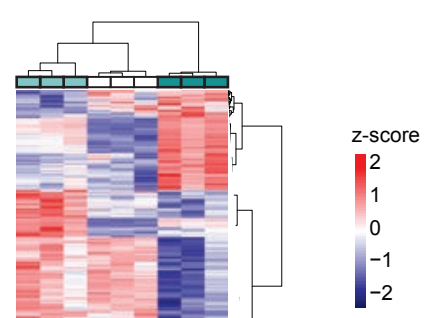

$\square \mathrm{CK}-\mathrm{p} 25 \mathrm{p} 65 \mathrm{kd}$

$\square$ CK PBS

$\square$ cK-p25 Scramble k Upregulated in CK-p25 p65 kd microglia vesicle-mediated transport in synapse cell-cell adh. via plasma-membrane adh mol synaptic vesicle mole synaptic vesicle cycle
synaptic transmission, glutamatergic
glutamate receptor signaling pathway regulation of membrane potential vesicle localization
multicellular organismal signaling adult behavior

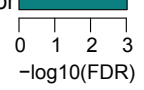

Downregulated in CK-p25 kd microglia cell killing

antigen processing and presentation adaptive immune response

negative regulation of cell activation

cell. response to lipoprotein particle stim.

regulation of innate immune response response to virus

leukocyte mediated immunity leukocyte apoptotic process

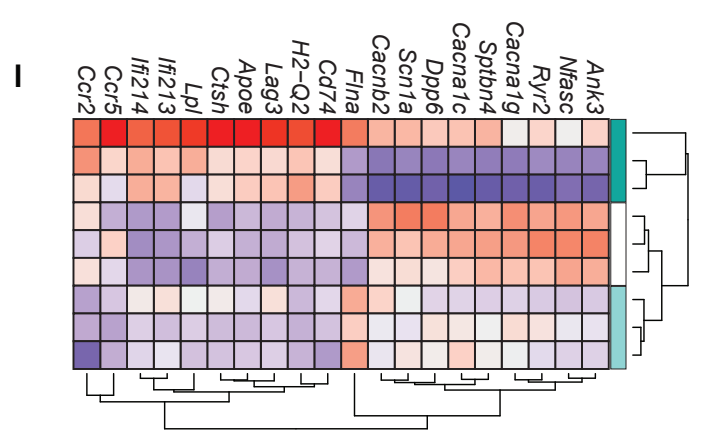

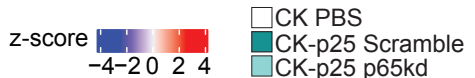

Figure 4. Immune signaling in neurons recruits and activates microglia.

a. Schematic of spatial transcriptomics experiment. CK $(n=3)$ and CK-p25 $(n=4)$ were induced for two weeks. Coronal brain sections were stained and imaged for $y$ H2AX then sequenced.

b. UMAP of capture areas from all samples. Leiden clusters are indicated by color and number. Each dot represents one capture area.

c. Leiden clusters superimposed onto a CK-p25 brain slice used for spatial transcriptomics.

d. UMAP indicating the density of $\mathrm{HH} 2 \mathrm{AX}$-positive capture areas.

e. $\mathrm{HH} 2 \mathrm{AX}$-positive capture areas identified in one CK-p25 brain slice. $\mathrm{YH} 2 \mathrm{AX}$-positive capture areas are shown in red. Captures are superimposed over DAPI staining (white).

f. Spatial clusters, yH2AX-positive capture areas, and reactive microglia signature gene expression in one CK-p25 sample.

g. Schematic of neuronal p65 knock-down experiment. CK-p25 mice receive retro-orbital injections of scramble shRNA-RFP AAV or shp65-RFP AAV. CK mice

received retro-orbital injections of PBS. Mice were allowed to recover for two weeks before being taken off dox. Brains were collected for subsequent analysis at the two week timepoint.

h. qRT-PCR of immune genes in sorted $\mathrm{yH} 2 \mathrm{AX}+$ nuclei.

i. Representative images of Iba1 immunostaining in CK, Scramble, and p65kd cortex.

j. Quantification of (left to right): Iba1+ soma area, number lba1+ per image, average branch length per Iba1+ cell, and number end-points per Iba1+ cell. Each data point represents one image. Two images were taken per mouse.

k. Heat map of differentially expressed genes from P65 vs. Scramble contrast. Each column represents one mouse.

I. Upregulated and downregulated gene ontology (biological pathway) terms identified through gene set enrichment analysis (GSEA) from p65 kd vs. scramble contrast.

m. Heatmap of significantly upregulated and downregulated genes in Pu.1+ nuclei from p65 kd cortex compared to Pu.1+ nuclei from scramble cortex. Each row represents gene expression from one mouse. 
50 uM Etoposide Vehicle Control $\longrightarrow$

50 uM Etoposide or Vehicle Control

+ NFkB Activation Inhibitor, $10 \mathrm{uM}$

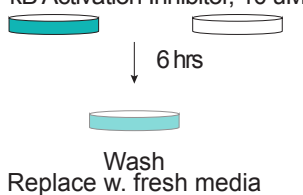

Replace w. fresh media

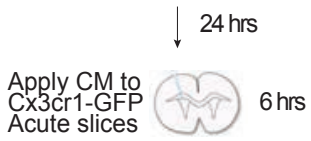

$6 \mathrm{hrs}$

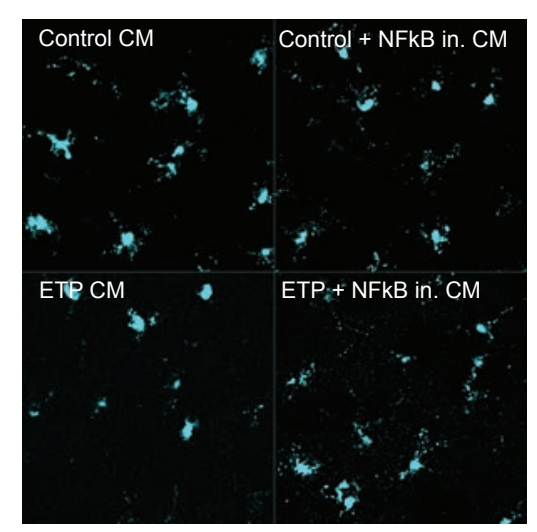

h $\quad \mathrm{Ccl} 2(\mathrm{pg} / \mathrm{ml})$ $\stackrel{\infty}{\circ}$

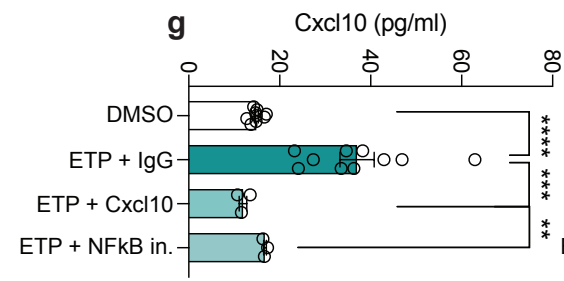

\section{j}

50 uM Etoposide Vehicle Control

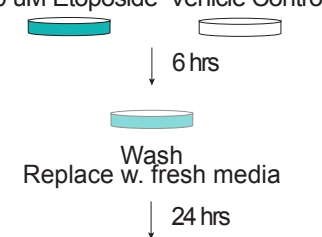

Ccl2 Immunodepletion

Cxcl10 Immunodepeltion

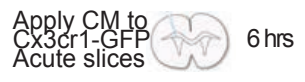

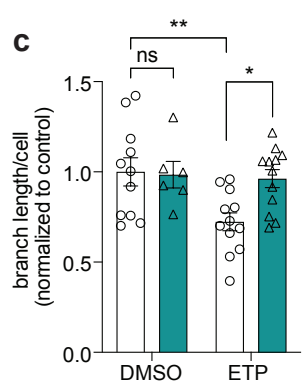
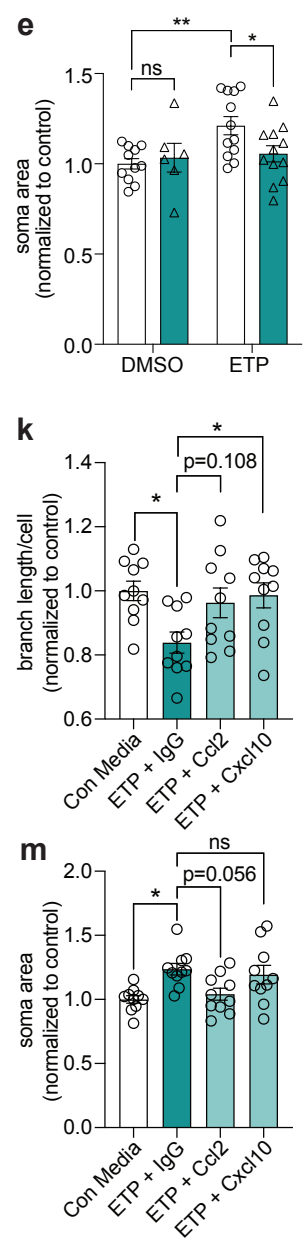

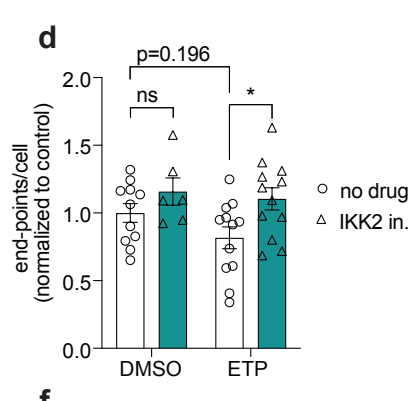

f
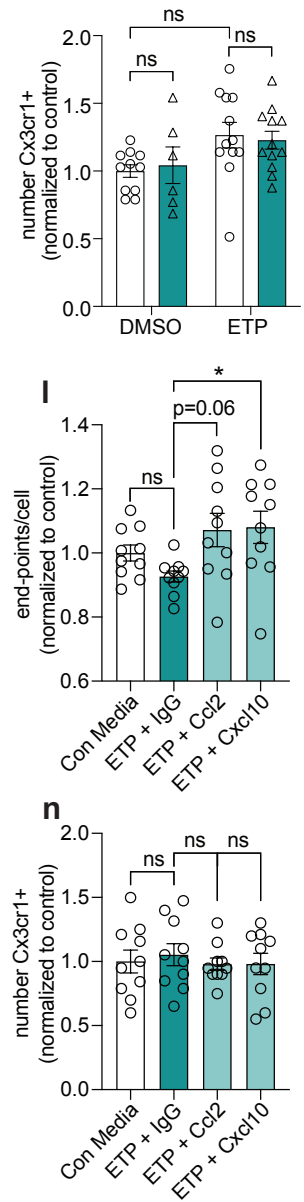

Figure 5. $\mathrm{Ccl} 2$ and $\mathrm{Cxcl} 10$ are secreted from DSB-bearing neurons to activate microglia.

a. Schematic for treating acute Cx3cr1-GFP slices with conditioned media from etoposide-treated primary neuron cultures. Cultures were either treated with 50uM ETP or vehicle control (DMSO) for six hours. In a separate condition, primary neurons were treated with 50uM ETP and 10uM NF-kappaB Activation Inhibitor VI (IKK2 inhibitor). Cultures were washed with PBS after six hours and media was replaced. After 24 hours, this media was applied to acute Cx3cr1-GFP slices for 6 hours.

b. Representative images of GFP in Cx3cr1-GFP acute slices treated with primary neuron conditioned media.

c-f. Quantification of branch length per microglia (c), end-points per microglia (d), soma area (e), and number of microglia per image (f). Each data point represents the average measurement from two images in one acute slice.

g,h. Quantification of Cxcl10 (g) and Ccl2 (h) from conditioned media from control and etoposide-treated primary neurons. Each datapoint represents one biological replicate.

i. Schematic of ETP conditioned media experiment. Primary neurons were treated with either ETP or DMSO for 6 hours, washed with PBS, then media was replaced. Cultures recovered for 24 hours before conditioned media was collected. IgG, Ccl2, or Cxcl10 antibodies were used to immunodeplete conditioned media before they were applied to Cx3cr1-GFP acute slices for 6 hours.

j. Representative images of microglia from acute slices treated with different conditioned media.

k-n. Quantification of $(k)$ branch length per microgli $(\mathrm{l})$ end-points per microglia, soma area $(\mathrm{m})$, and number of microglia per image $(\mathrm{n})$. Each data point represents the average measurement from two images in one acute slice.

Error bars represent standard error of mean (S.E.M.); ${ }^{* * *} \mathrm{P}<0.0001,{ }^{* *} \mathrm{P}<0.001,{ }^{* *} \mathrm{P}<0.01,{ }^{*} \mathrm{P}<0.05$, ns not significant. Two-way ANOVA followed by Sidak's test for multiple comparisons (c-f). One-way ANOVA followed by Tukey's test for multiple comparisons (k-n). Data are combined from two independent experiments (c-f, k-n). Data are combined from three independent experiments $(h, i)$. 
a
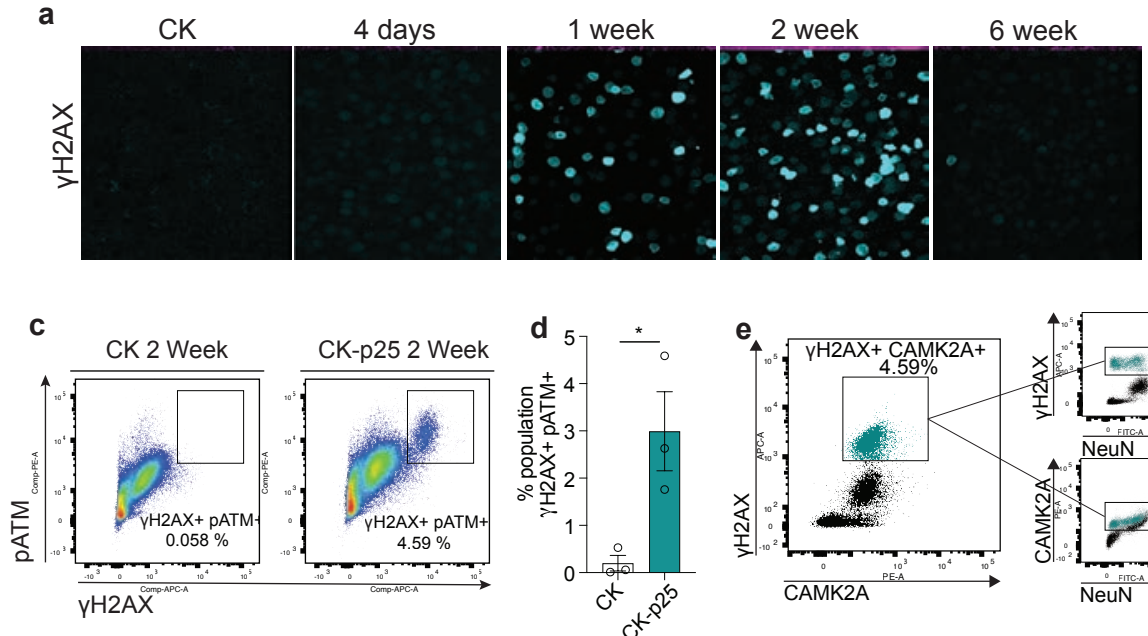

b
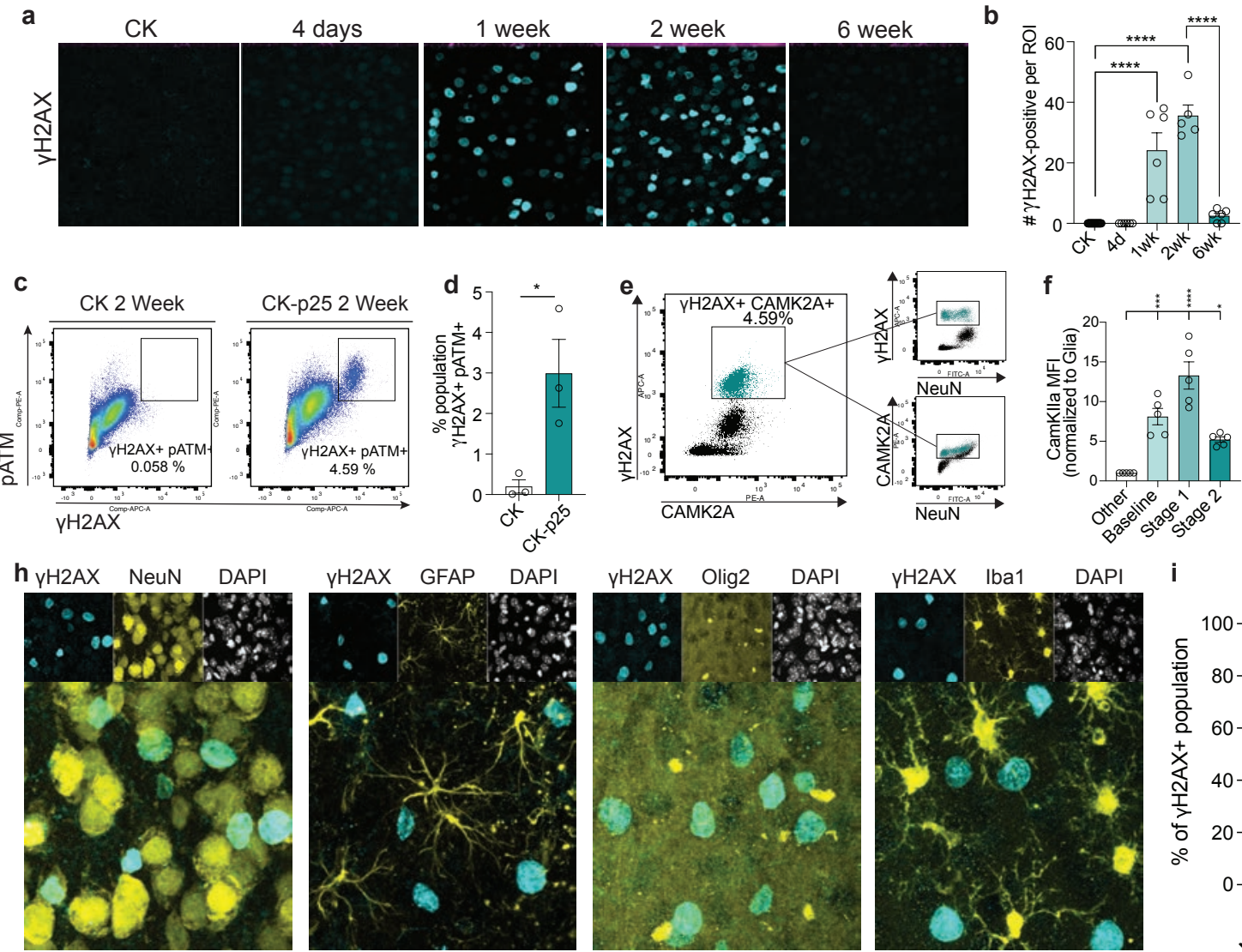

YH2AX/NeuN Merge

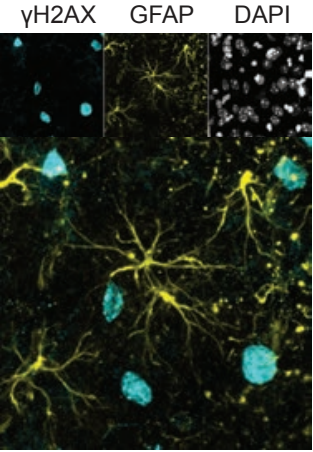

YH2AX/GFAP Merge

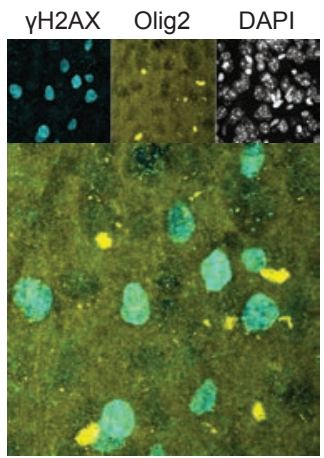

yH2AX/Olig2 Merge
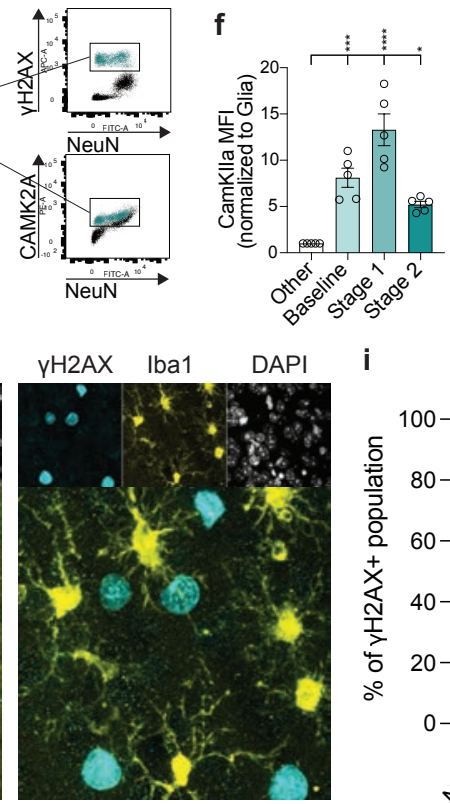

YH2AX/lba1 Merge
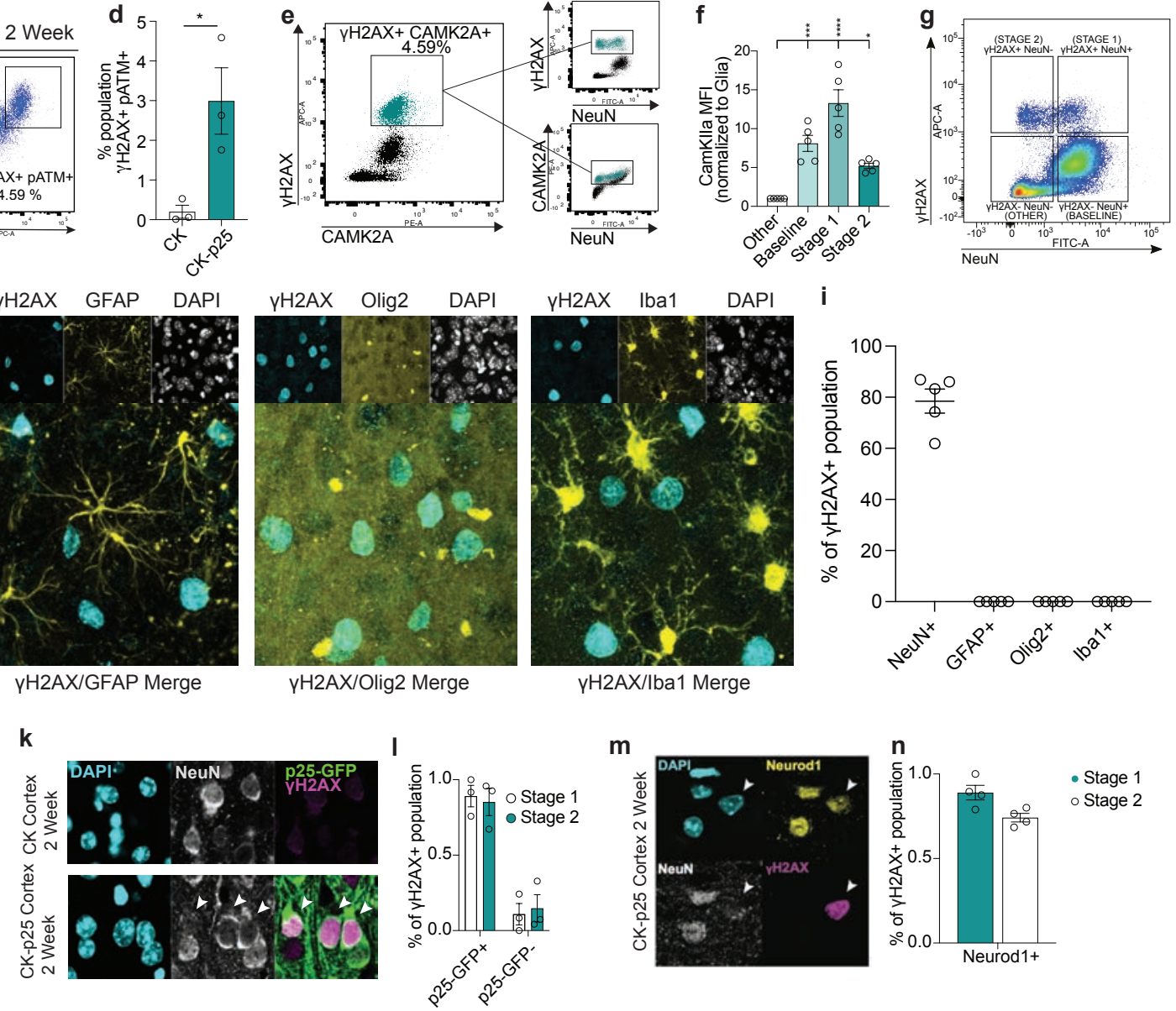

m
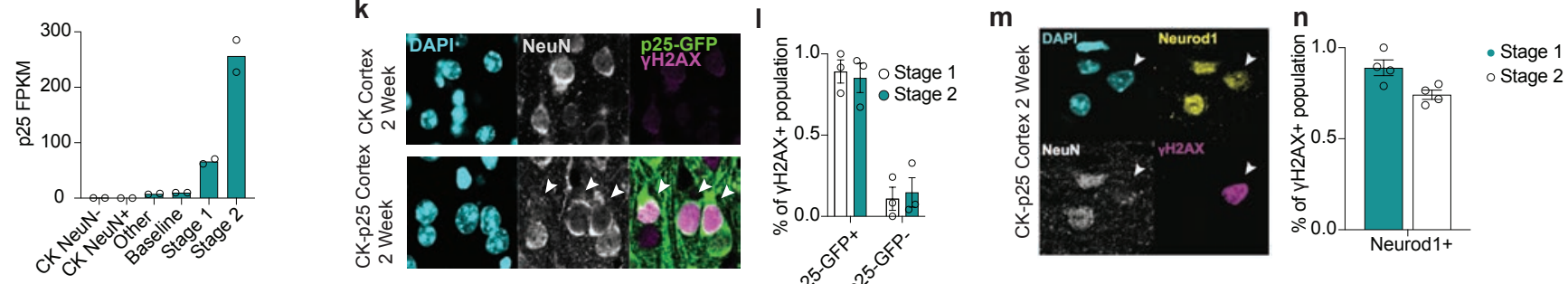

\section{Supplementary Figure 1.}

a. Representative images of $\mathrm{yH} 2 \mathrm{AX}$ immunostaining in $\mathrm{CK}$ and $\mathrm{CK}-\mathrm{p} 25$ cortex over a 6-week timeline analysis

b. Average number of $\mathrm{yH} 2 \mathrm{AX}+$ nuclei quantified per image at each timepoint. Each data point represents one mouse.

c. Flow cytometry dot plot of $\mathrm{YH} 2 \mathrm{AX}+\mathrm{pATM}+$ nuclei in $\mathrm{CK}$ and CK-p25 cortex.

d. Quantificaton of $\mathrm{yH} 2 \mathrm{AX}+\mathrm{pATM}+$ nuclei in $\mathrm{CK}$ and $\mathrm{CK}-\mathrm{p} 25$ cortex. Each data point represents percent $\mathrm{yH} 2 \mathrm{AX}+\mathrm{pATM}+$ nuclei for one mouse.

e. Flow cytometry dot plot of $\mathrm{yH} 2 \mathrm{AX}$, Camk2a, and NeuN immunoreactivity in nuclei from CK-p25 cortex. yH2AX+ Camk2a+ nuclei are gated in the left graph. This gated population is highlighted in turquoise in the two graphs to the right.

f. Camk2a median fluorescent intensity (MFI) is quanitifed for Glia, Baseline, Stage 1, and Stage 2 populations. MFI values are normalized to the Glia population.

g. Representative dot plot of $\mathrm{\gamma H} 2 \mathrm{AX}$ and NeuN immunoreactivity in 2-week induced CK-p25 cortex.

h. Representative images of cell type and $\mathrm{yH} 2 \mathrm{AX}$ immunostaining in the 2 wk-induced CK-p25 cortex. Celltype markers from left to right: neurons

(NeuN), astrocytes (GFAP), oligodendrocytes and oligodendrocyte precursor cells (Olig2), microglia (Iba1).

i. Quantification of percent of $\mathrm{yH} 2 \mathrm{AX}$-positive nuclei overlapping with each celltype stain. Each dot represents one mouse. $50-100 \mathrm{yH} 2 \mathrm{AX}-$ positive nuclei were analyzed for each mouse and celltype marker.

j. Expression p25 in CK and CK-p25 gated populations. One datapoint represents fragments per kilobase per million (FPKM) from a gated population from one mouse.

k. Representative images of GFP, $y \mathrm{H} 2 \mathrm{AX}$, and NeuN immunostaining from CK and CK-p25 cortex at the 2wk time point. Arrowheads indicate $\mathrm{Y}$

$\mathrm{H} 2 \mathrm{AX}+$ nuclei that express $\mathrm{p} 25-\mathrm{GFP}$ regardless of NeuN immunoreactivity.

I. Quantification of GFP expression across Stage 1 and Stage 2 populations. Each data point represents percent $\mathrm{yH} 2 \mathrm{AX}+$ population from Stage 1 or Stage 2 from one CK-p25 mouse.

m. Representative images of Neurod1, $\mathrm{yH} 2 \mathrm{AX}$, and NeuN from CK-p25 cortex at two-week timepoint. White arrowhead indicates a yH2AX+ nucleus with Neurod1 immunoreactivity but not NeuN immunoreactivity.

n. Quantification of Neurod1 expression across Stage 1 and Stage 2 populations. Each data point represents percent $y H 2 A X+$ population from Stage 1 or Stage 2 from one CK-p25 mouse.

Error bars represent standard error of mean (S.E.M.); ${ }^{* * *} P<0.0001$, ${ }^{* * *} P<0.001,{ }^{*} P<0.05$, n.s. not significant; One-way ANOVA with Tukey's test for multiple comparisons $(b, f)$. Student's T-test $(d)$. Data are averages of 4 images per mouse $(b)$. Data are representative of at least 2 independent experiments (d). Data are pooled from 2 independent experiments (f). 
b Stage 1 vs Baseline

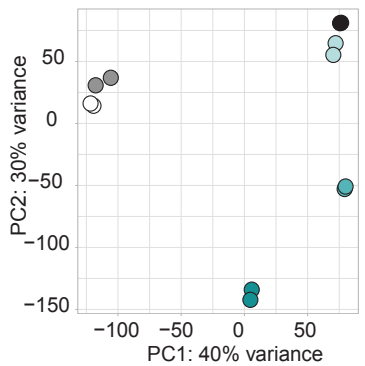

- CK NeuN+ O CK-p25 Baseline

- CK NeuN- O CK-p25 Other

O Stage 2

Stage 1
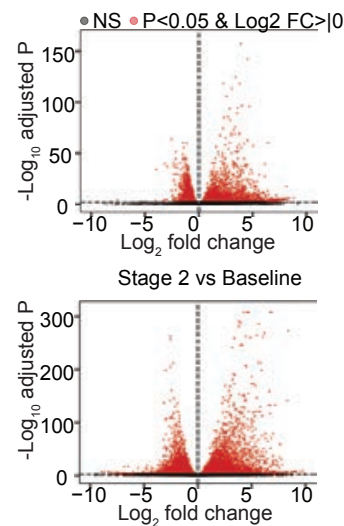

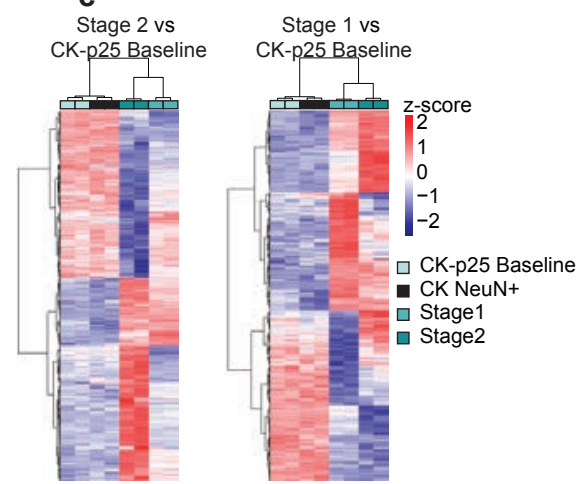

d
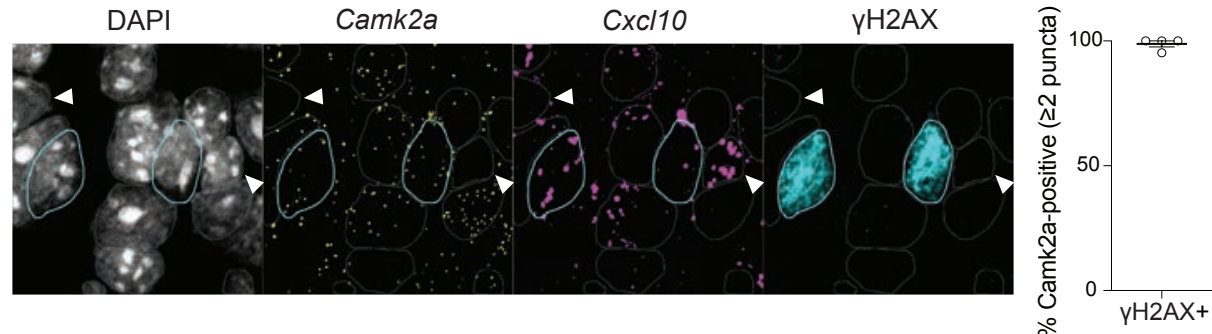

e
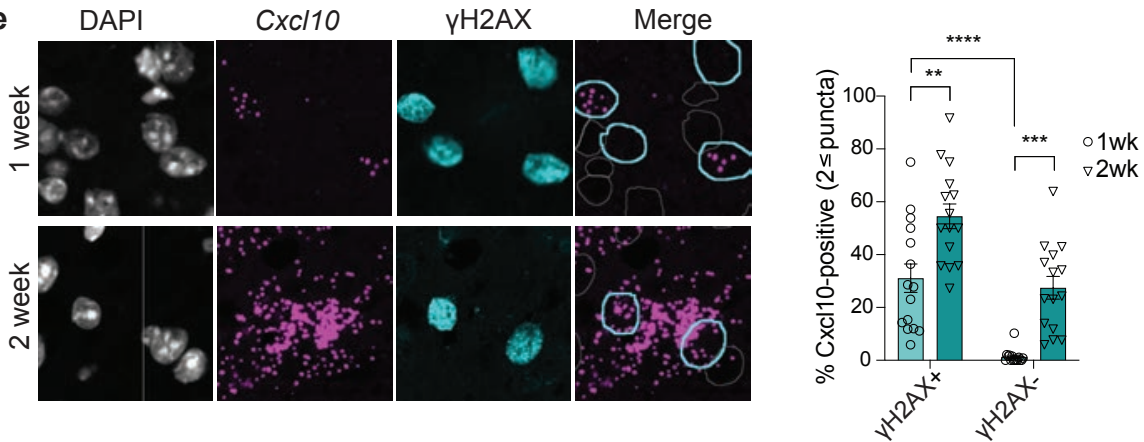

f

K-p25

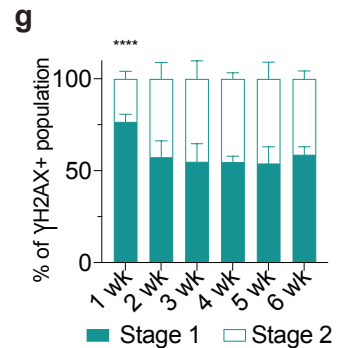

\section{Supplementary Figure 2.}

a. Principle component analysis (PCA) of normalized gene expression matrix from CK and CK-p25 subpopulations. Principle component 1 (PC1): $66 \%$ variance. Principle component 2 (PC2): 29\% variance.

b. Volcano plots of (top) Stage 1 vs. CK-p25 Baseline and (bottom) Stage 2 vs. CK-p25 Baseline contrasts. Gray circles: non-significant (ns) transcripts. Red circles: transcripts with FDR adjusted $p$-value $<0.05$ and $\log _{2}$ fold change $>|0.1|$.

c. Heatmap of differentially expressed genes from Stage 2 vs. CK-p25 Baseline and Stage 1 vs. CK-p25 Baseline contrasts. Each column represents one mouse.

d. Representative image of RNAscope probes for Camk2a (yellow) and Cxcl10 (magenta), and $\mathrm{yH} 2 \mathrm{AX}$ immunostaining (turquoise). To the right, the percent of $\mathrm{Y}$ H2AX-positive cells that are Camk2a-positive (2 or more Camk2a puncta within the nucleus) are quantified. Each datapoint represents one 2-week induced CK-p25 mouse. 17-42 $\mathrm{yH} 2 \mathrm{AX}$-positive cells were quantified for each mouse. Turquoise outlines indicate yH2AX-positive Camk2a-positive cells. Gray outlines indicate $\mathrm{Y}$ H2AX-negative cells. White arrows indicate Camk2a-negative cells.

e. Representative image of the RNAscope probe for $\mathrm{Cxcl10}$ (magenta) combined with $\mathrm{YH} 2 \mathrm{AX}$ immunostaining (turquoise). Imaging was performed on 1 wk and 2wk CK-p25 cortices. To the right, the number of $\mathrm{yH} 2 \mathrm{AX}$-positive and $\mathrm{yH} 2 \mathrm{AX}$-negative cells with 2 or more $\mathrm{Cxcl10}$ puncta are quantified. Each datapoint represents the average \% Cxcl10-positive cells in one image from one mouse. 4-3 images were taken per mouse. (CK-p25 1wk n=4, CK-p25 2wk n=4).

f. Representative dot plots of $\mathrm{yH} 2 \mathrm{AX}$ and NeuN immunoreactivity in CK and CK-p25 mice at 1, 2, and 6 weeks induction. Stage 1 and Stage 2 percent population was calculated with respect to the total $\mathrm{YH} 2 \mathrm{AX}$-positive population.

g. Stage 1 and Stage 2 percent population are quantified for each timepoint.

Error bars represent standard error of mean (S.E.M.); ${ }^{* * *} \mathrm{P}<0.0001,{ }^{* * *} \mathrm{P}<0.001,{ }^{* *} \mathrm{P}<0.01$, ${ }^{*} \mathrm{P}<0.05$, n.s. not significant; Two-way ANOVA followed by Sidak's test for multiple comparisons $(e, g)$. Data are representative of 2 independent experiments (d). Data are pooled from four independent experiments $(g)$. 


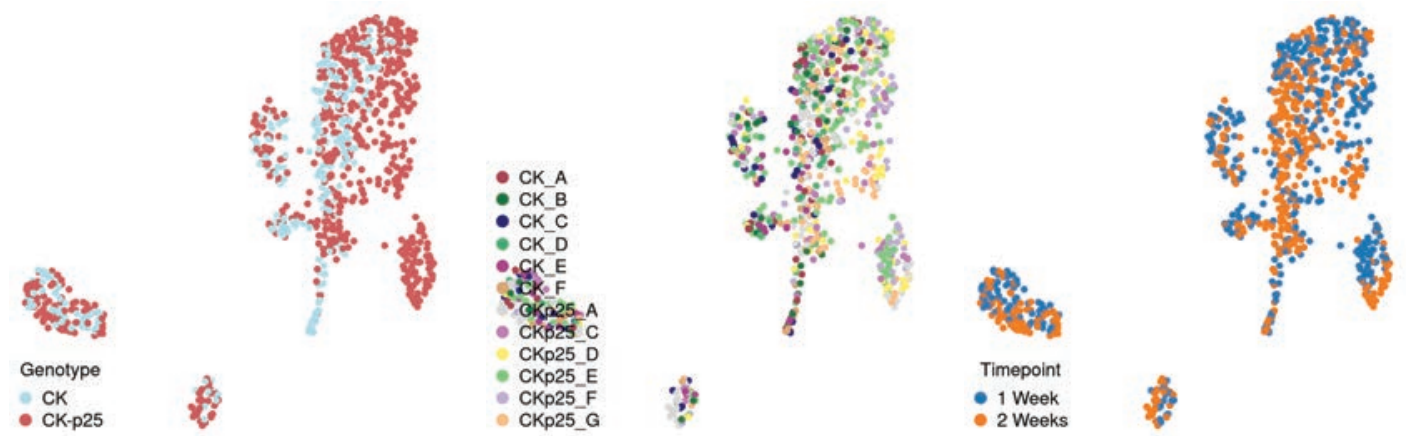

b

Nuclei FANS-gated as "Stage 2"

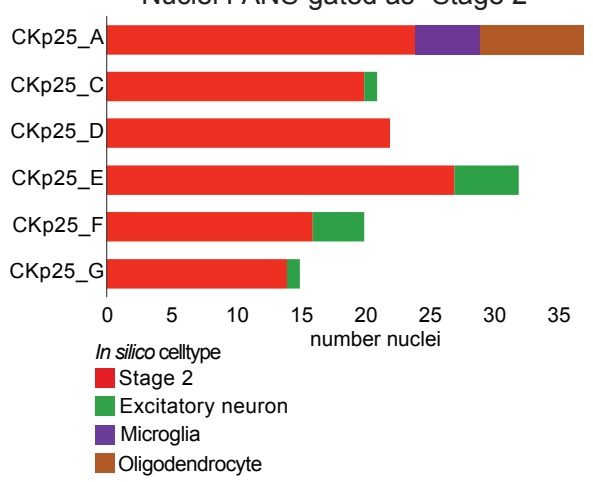

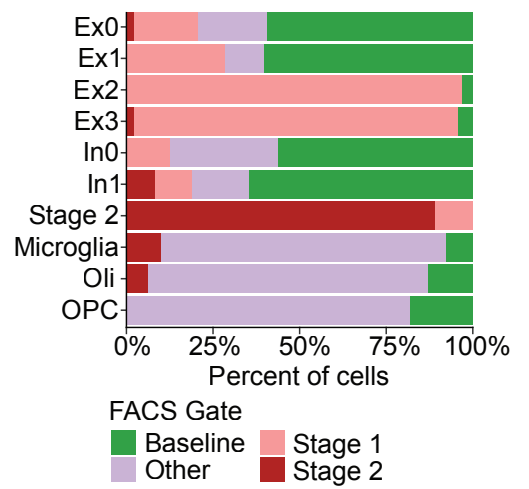

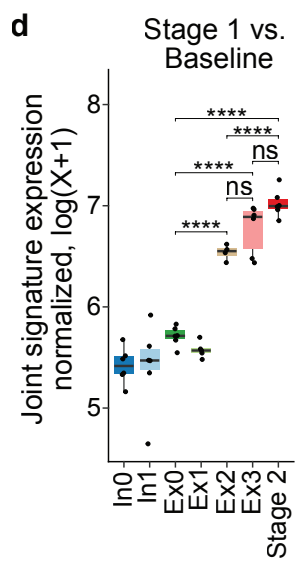

Stage 2 vs. Immune genes

Baseline enriched in Stage 2

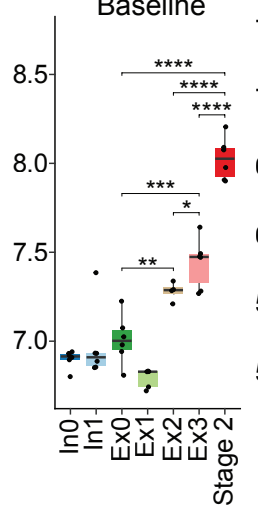
.5-

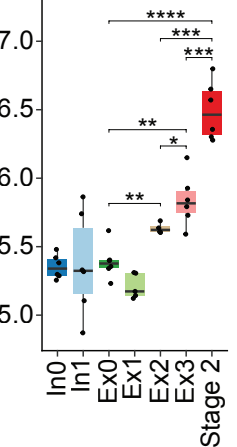

\section{Supplementary Figure 3.}

a. UMAPs labeled by genotype, mouse id, and timepoint.

b. Distribution of Stage 2-gated nuclei in in silico cell type clusters, stratified by biological replicate.

c. Percent FANS label distribution across scRNA cell type clusters. Bargraph colors refer to FANS gate label.

d. Bulk RNA-seq gene signature enrichment in neuron cell type clusters. Stage 1 signature enrichment (left), Stage 2 signature enrichment (middle), and immune genes from Stage 2 enrichment (right). Each datapoint indicates average gene expression across all cells from one mouse. Only CK-p25 mice were used for this analysis. 


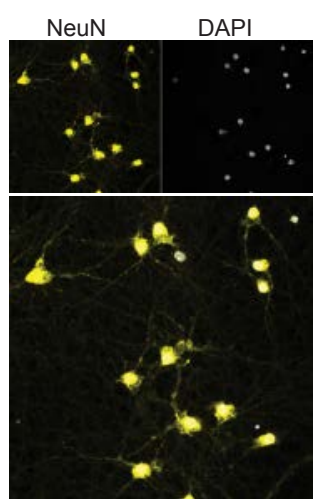

NeuN/DAPI Merge
GFAP

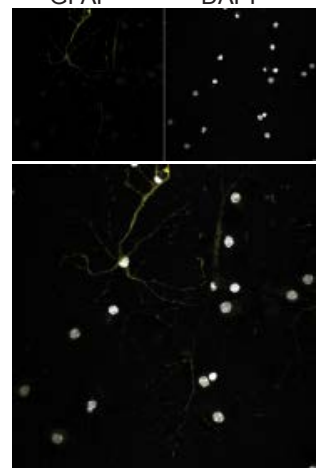

GFAP/DAPI Merge

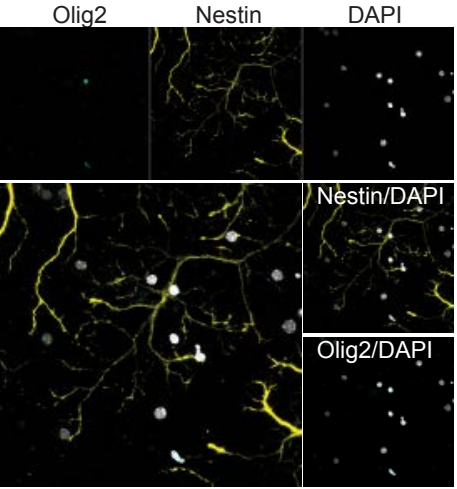

Olig2/Nestin/DAPI Merge

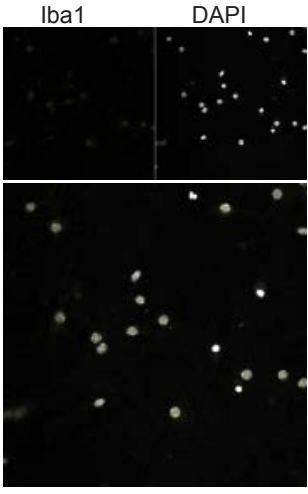

Iba1/DAPI Merge

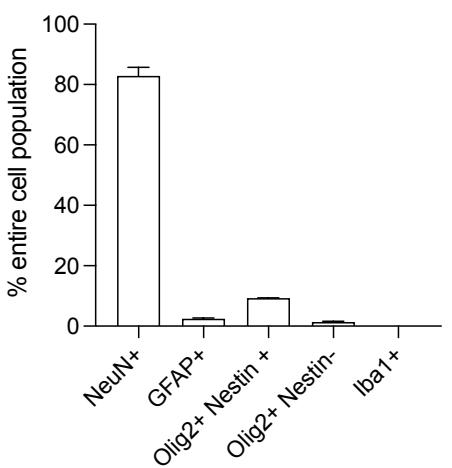

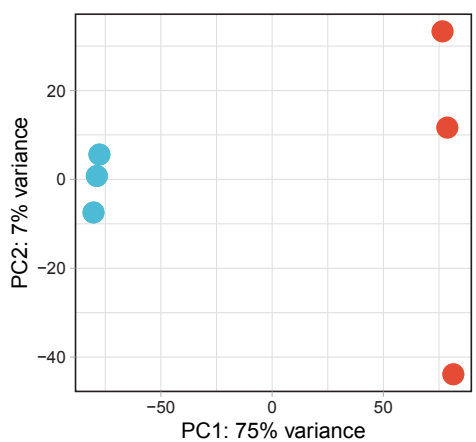

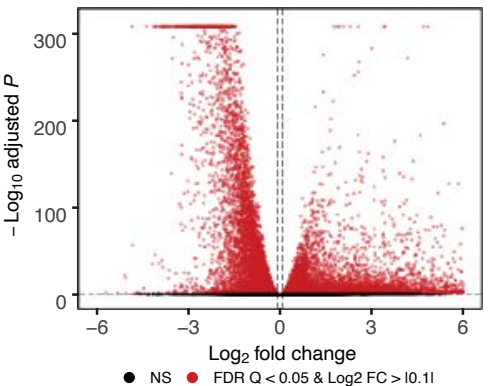

d

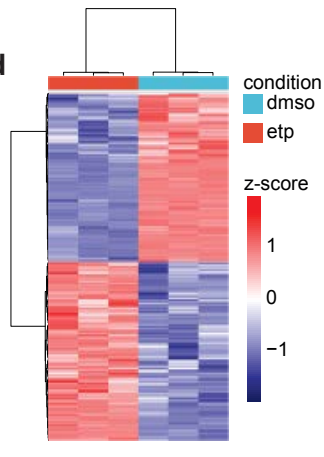

e

f
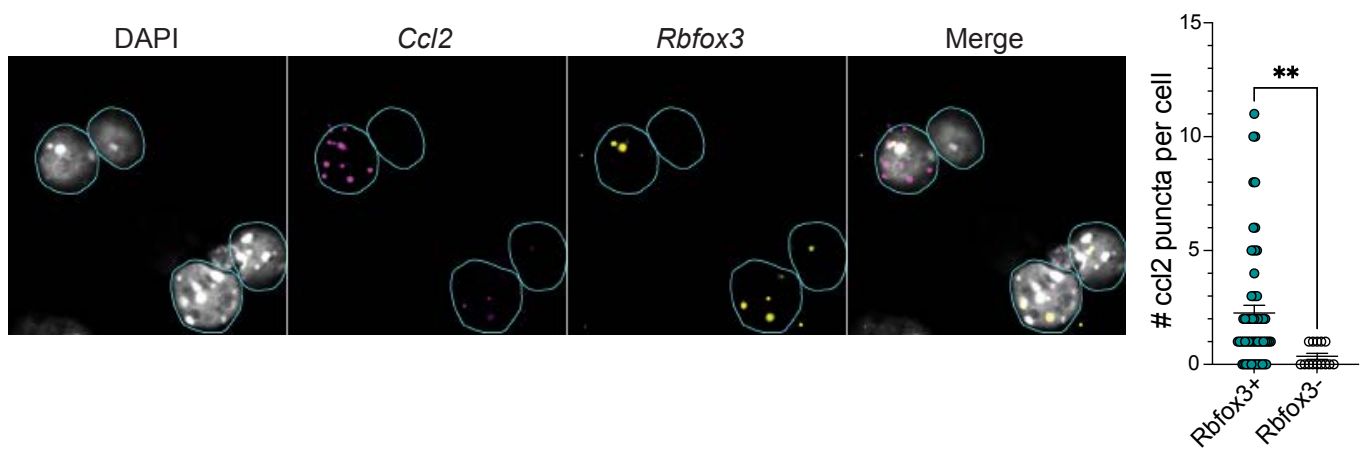

\section{Supplementary Figure 4.}

a. Representative images of celltype immunostaining in DIV13 primary neuron cultlures. The percent nuclei that stained positively for each celltype marker are quantified on the right. Three images were analyzed for each marker.

b. PCA plot of normalized gene expression matrix from ETP and DMSO-treated neurons. Principle component 1 (PC1): $75 \%$ variance. Principle component 2 (PC2): $7 \%$ variance.

c. Volcano plot of ETP vs. DMSO contrast. Gray circles: non-significant (ns) transcripts. Red circles: transcripts with FDR adjusted p-value <0.05 and log ${ }_{2}$ fold change $>|0.1|$.

d. Heatmap of differentially expressed genes from ETP vs. DMSO contrast. Columns represent biological replicates.

e. Quantification of Stage 1 and Stage 2 signature enrichment (from bulk RNAseq) in DMSO and ETP-treated neurons. Each data point represents one biological replicate.

f. Number of $\mathrm{Ccl} 2$ puncta (magenta) are quantified for Rbfox3-positive nuclei (2 or more Rbfox3 puncta within or surrounding the nucleus, yellow), and Rbfox3-negative nuclei. Cyan circles outline nuclei. Each datapoint represents one nucleus.

Error bars represent standard error of mean (S.E.M.); ${ }^{* * *} \mathrm{P}<0.0001,{ }^{* * *} \mathrm{P}<0.001,{ }^{*} \mathrm{P}<0.05$. Wilcoxon test (e). Student's t-test (f). Data are representative of two independent experiments $(\mathrm{a}, \mathrm{f})$. 


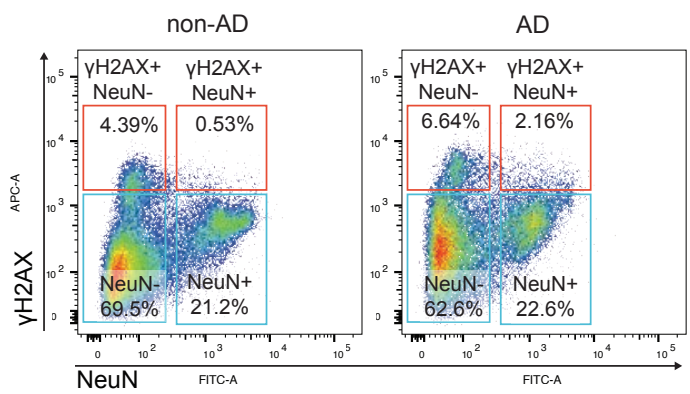

b

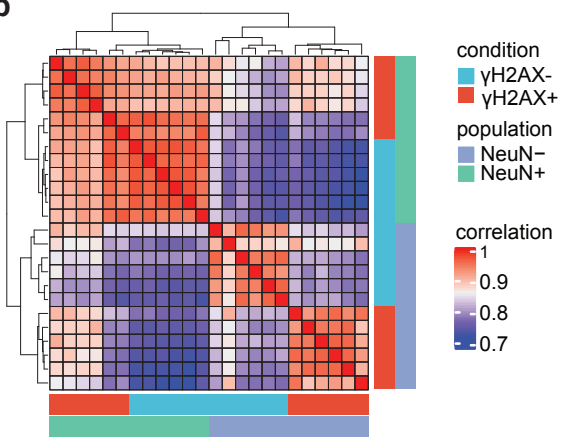

c

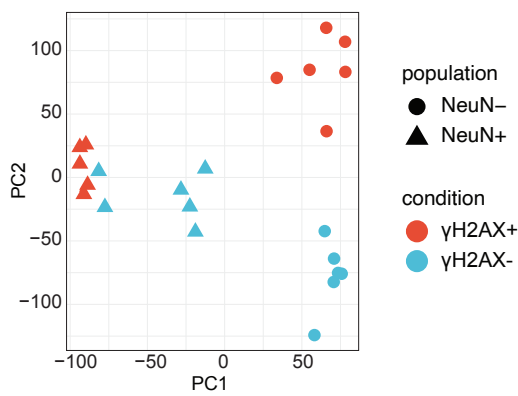

d

Neuronal

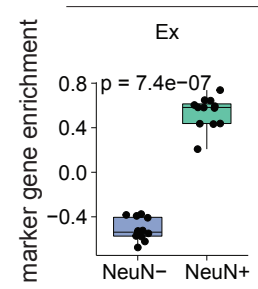

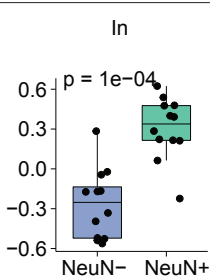
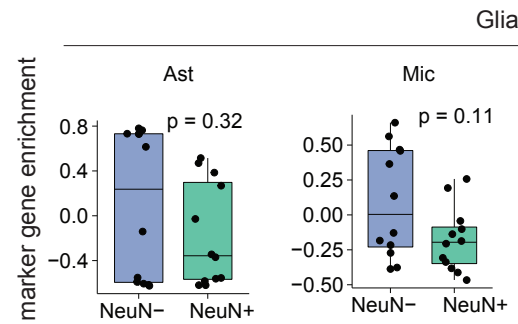

Glial
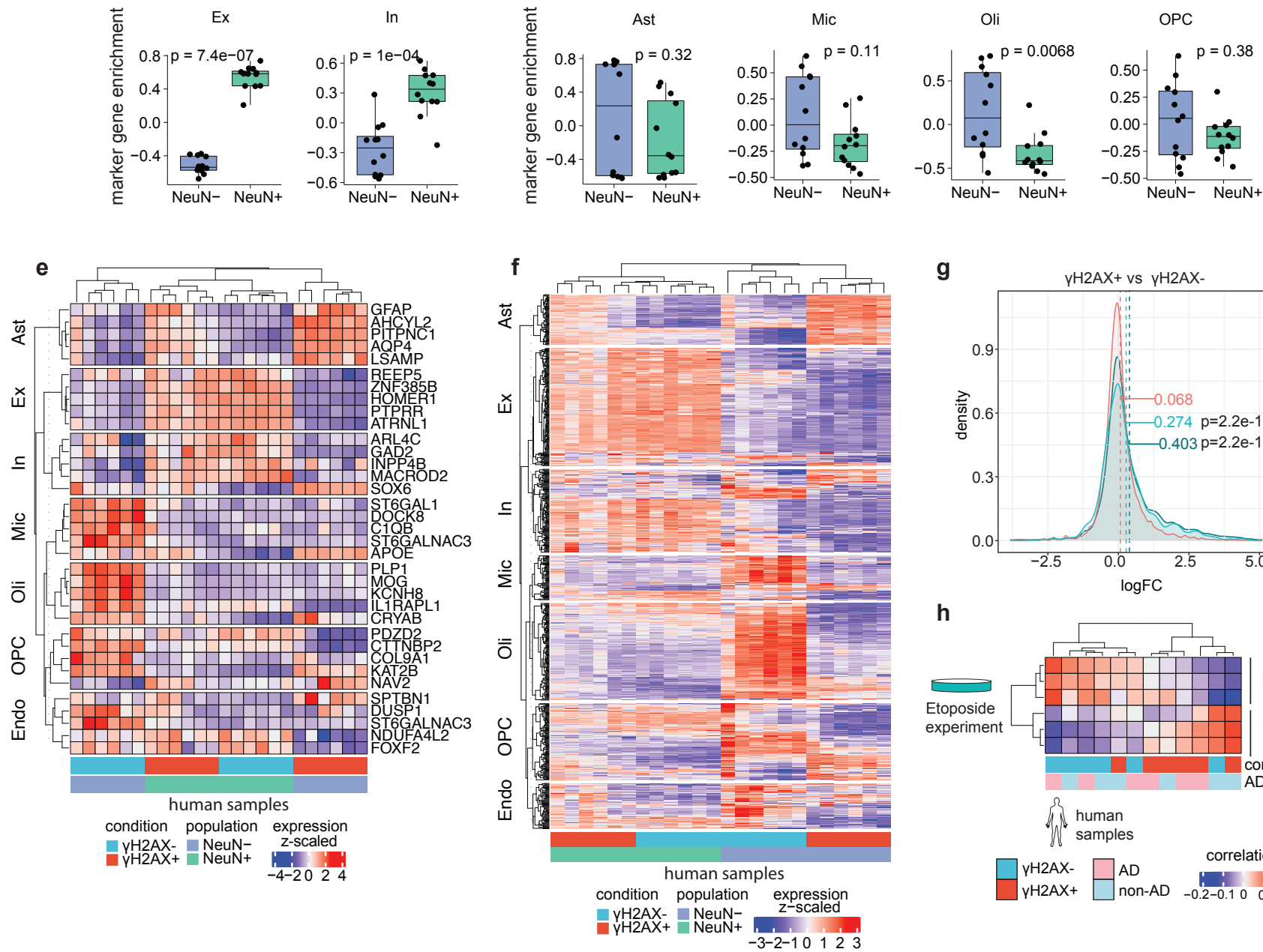

g

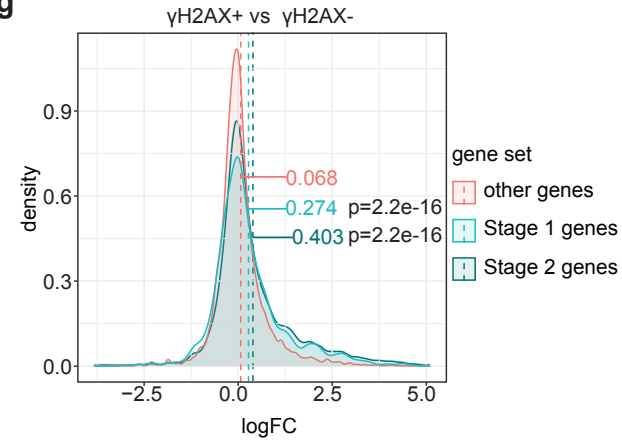

h

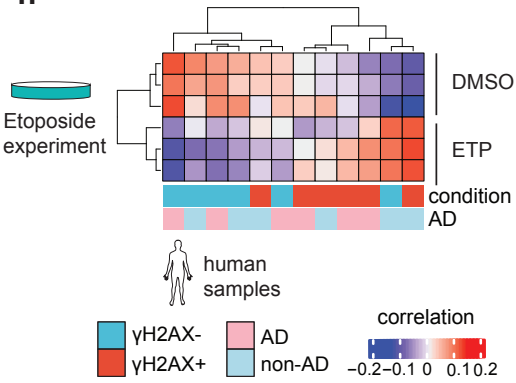

\section{Supplementary Figure 5.}

a. Flow cytometry dot plot of $\mathrm{yH} 2 \mathrm{AX}$ gating for human $\mathrm{NeuN}+$ and $\mathrm{NeuN}$ - nuclei. Percent total population is indicated for each gating box.

b. Correlation heatmap of $\mathrm{yH} 2 \mathrm{AX}+$ and $\mathrm{yH} 2 \mathrm{AX}-/ \mathrm{NeuN}+$ and NeuN- bulk RNAseq libraries from postmortem temporal cortex.

c. PCA plot of normalized gene expression from $\mathrm{yH} 2 \mathrm{AX}+$ and $\mathrm{yH} 2 \mathrm{AX}-/ \mathrm{NeuN}+$ and $\mathrm{NeuN}-$ samples.

d. Quantification of normalized neuronal and glial marker gene expression in $\mathrm{NeuN}+$ and NeuN-samples. Mean gene expression is compared with a Wilcoxon test. Gene set enrichment was calculated using Gene Set Variation Analysis (GSVA).

e. Gene expression heatmap of top five marker genes for major brain cell types. Each column represents one human sample.

f. Gene expression heatmap of all marker genes for major brain cell types. Each column represents one human sample.

g. Distribution of logFC values for Stage 1 genes (light blue), Stage 2 genes (dark blue), and other genes (red). LogFC values are from $y H 2 A X+v s ~ y H 2 A X-$ comparison. Mean LogFC for each gene set are compared with a Wilcoxon test.

h. Heatmap of ETP and $\mathrm{yH} 2 \mathrm{AX}+$ and $\mathrm{yH} 2 \mathrm{AX}$ - human neuronal nuclei transcriptional correlation.

Excitatory (Ex), Inihibtory (In), Astrocyte (Ast), Microglia (Mic), Oligodendrocyte (Oli), Oligodendrocyte Precursor Cells (OPC), Endothelial (Endo). 
a

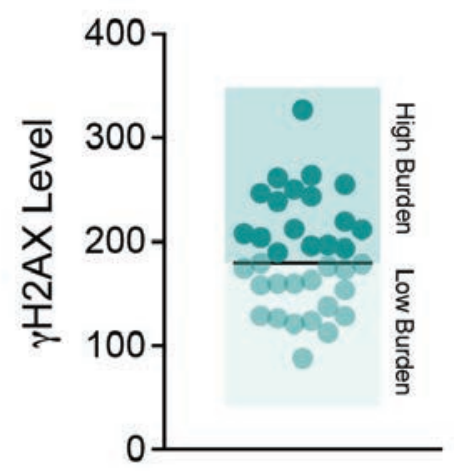

NeuN-Positive Cell

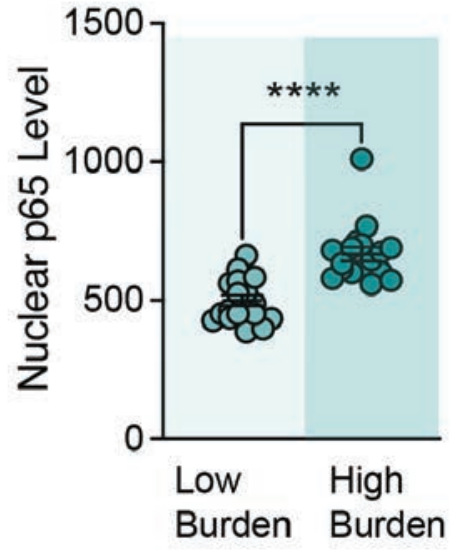

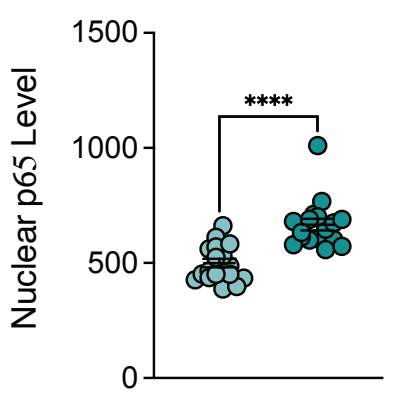
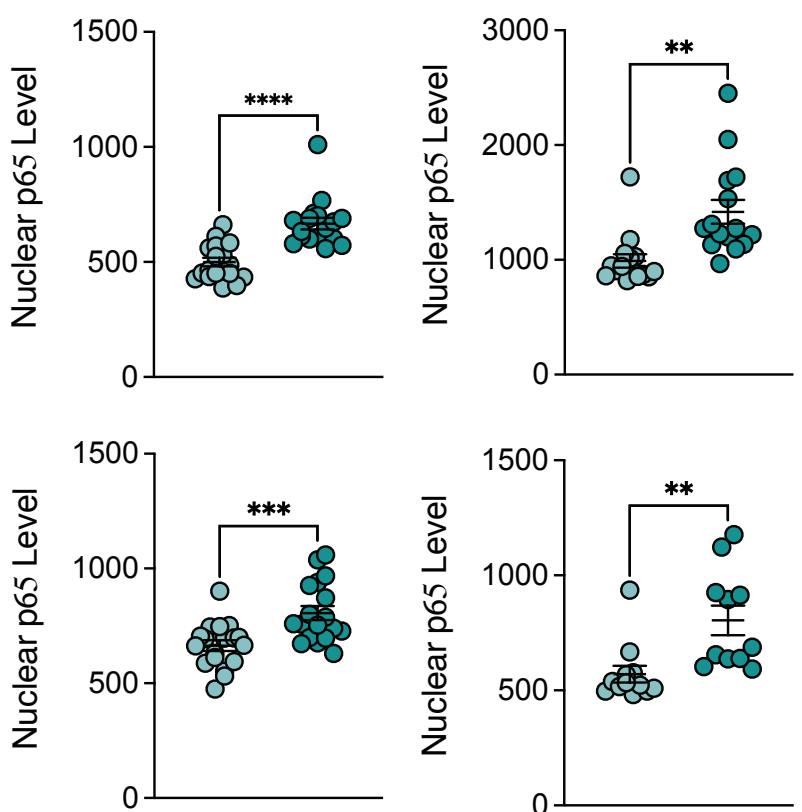

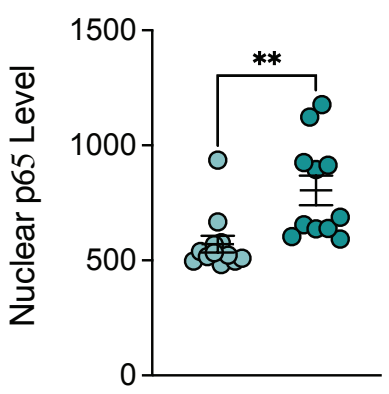

\section{Supplementary Figure 6.}

a. Schematic for binning $\mathrm{YH} 2 \mathrm{AX}$ expression in NeuN-positive cells, and nuclear p65 quanitification. For each individual, the median $\mathrm{YH} 2 \mathrm{AX}$ expression in NeuN-positive cells was calculated. Cells were then binned as $\mathrm{yH} 2 \mathrm{AX}$ high or $\mathrm{yH} 2 \mathrm{AX}$ low based off of median $\mathrm{yH} 2 \mathrm{AX}$ expression. Nuclear p65 intensity was then calculated for all cells.

b. Raw data from the four $A D$ individuals used for analysis.

Error bars represent standard error of mean (S.E.M.); ${ }^{* * *} \mathrm{P}<0.0001,{ }^{* * *} \mathrm{P}<0.001,{ }^{* *} \mathrm{P}<0.01,{ }^{*} \mathrm{P}<0.05$, ns not significant. A t-test was performed to compare mean p65 intensity between $\mathrm{yH} 2 \mathrm{AX}$ low and $\mathrm{yH} 2 \mathrm{AX}$ high cells. 
a

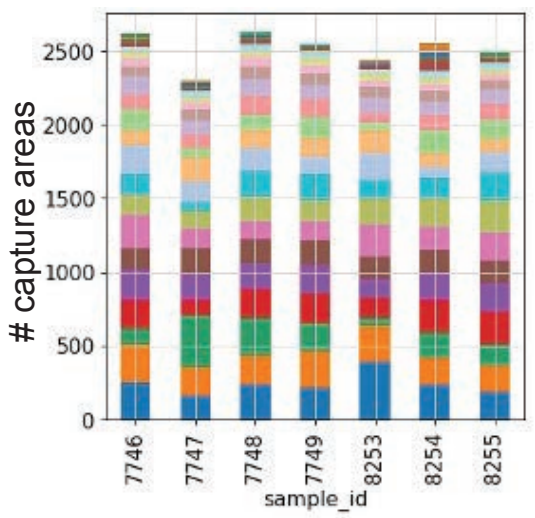

leiden clusters

$\begin{array}{llllll}0 & 1 & 2 & 3 & 4 & 5\end{array}$

10000

$\begin{array}{lllllll}6 & 7 & 8 & 9 & 10 & 11\end{array}$

1214151617

(2)

1819202122

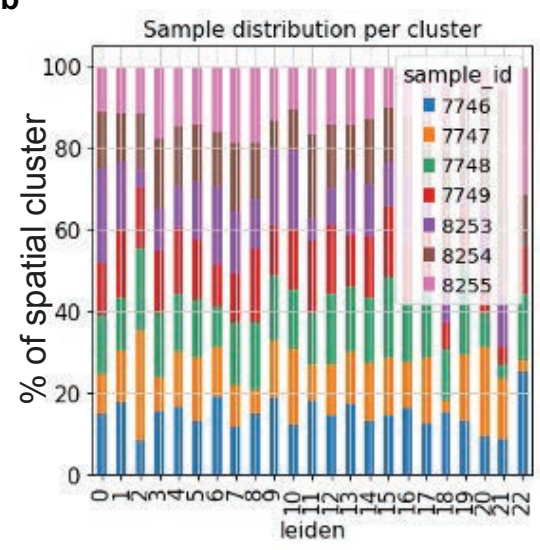

C 2-week CK-p25 Sections for Visium Spatial Gene Expression
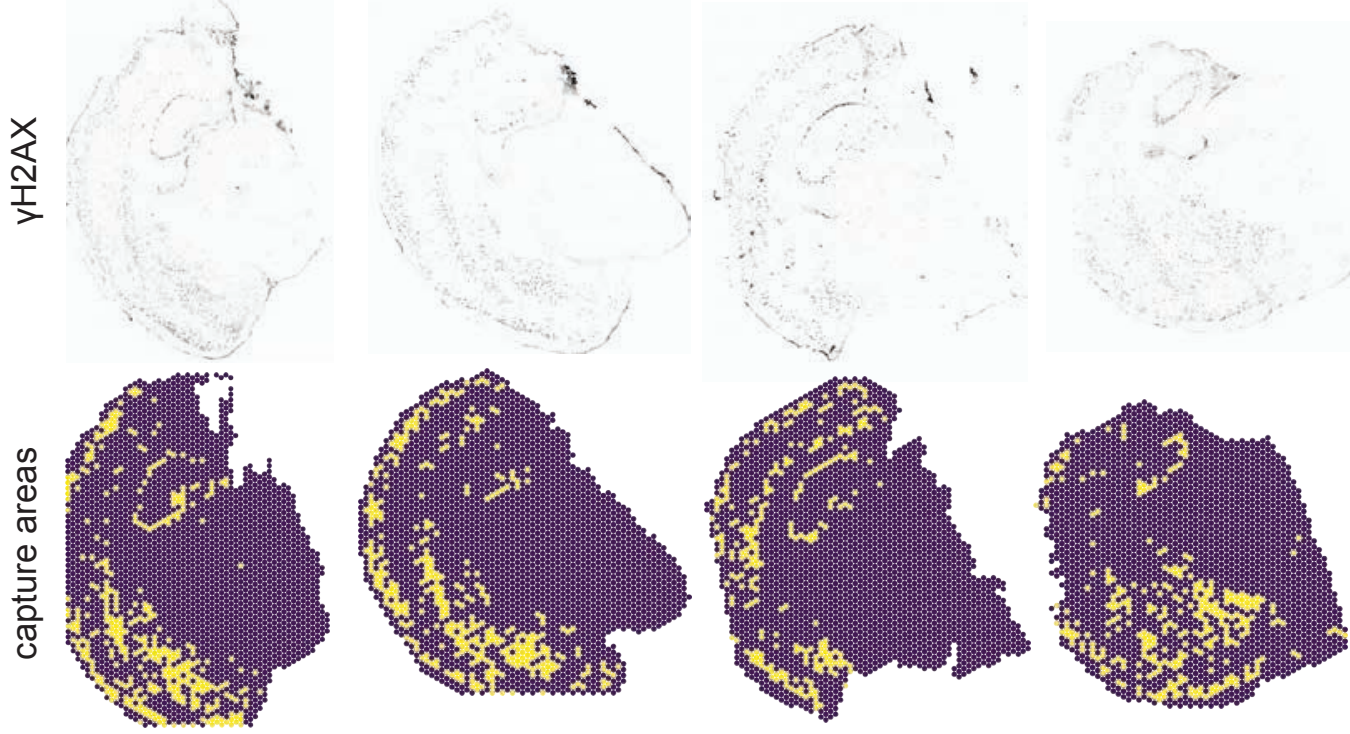

Supplementary Figure 7.

a. Distrubution of capture areas amongst spatial clusters for each sample. The y-axis refers to number capture areas. Colors refer to spatial cluster id.

b. Distribution of samples by spatial cluster. Colors refer to sample id.

c. Top: $\mathrm{yH} 2 \mathrm{AX}$ immunostaining for each CK-p25 section. Bottom: the corresponding capture areas identified as $\mathrm{yH} 2 \mathrm{AX}$-positive are marked in yellow. $\mathrm{\gamma H} 2 \mathrm{AX}$-negative capture areas are marked in purple. 
Mathys et al., 2017 microglia signature enrichment
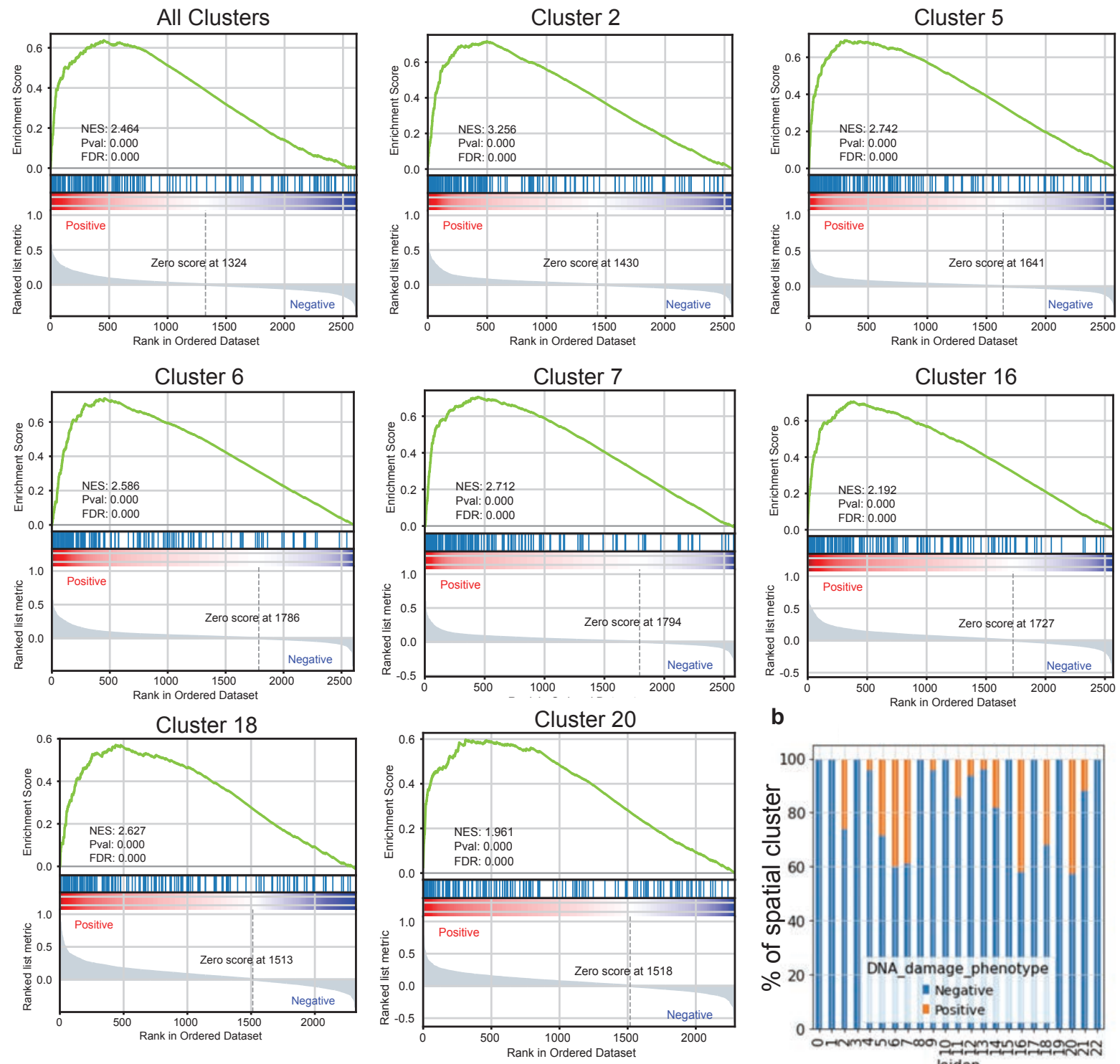

b

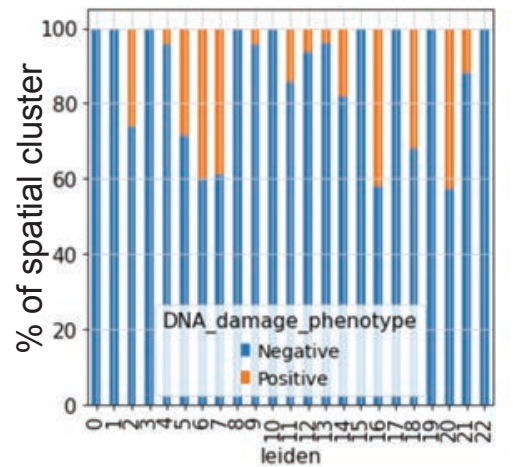

\section{Supplementary Figure 8.}

a. Gene set enrichment analysis of $\mathrm{YH} 2 \mathrm{AX}$-positive capture area DEGs. DEGs were tested for the enrichment of the reactive microglia signature characterized in Mathys et al., 2017. The analysis was first performed for all yH2AX-posistive capture areas, then for individual clusters comprised of $20 \%$ or more $\mathrm{yH} 2 \mathrm{AX}$-positive capture areas. Normalized enrichment score (NES).

b. Percent $\mathrm{YH} 2 \mathrm{AX}$-positive capture areas by spatial cluster. Orange indicates $\mathrm{yH} 2 \mathrm{AX}$-postive capture areas. Blue indicates pH2AX-negative capture areas. 


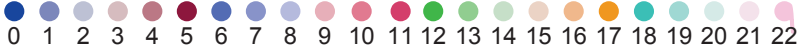

CK
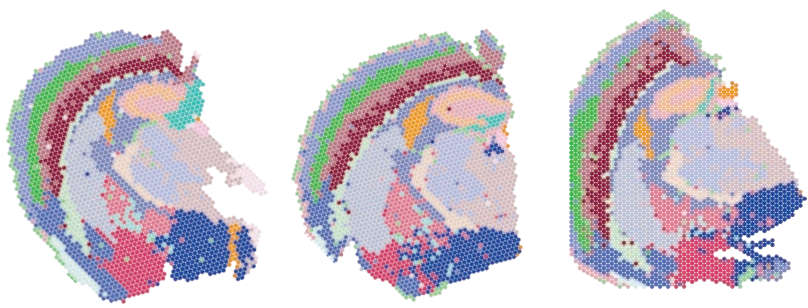

b

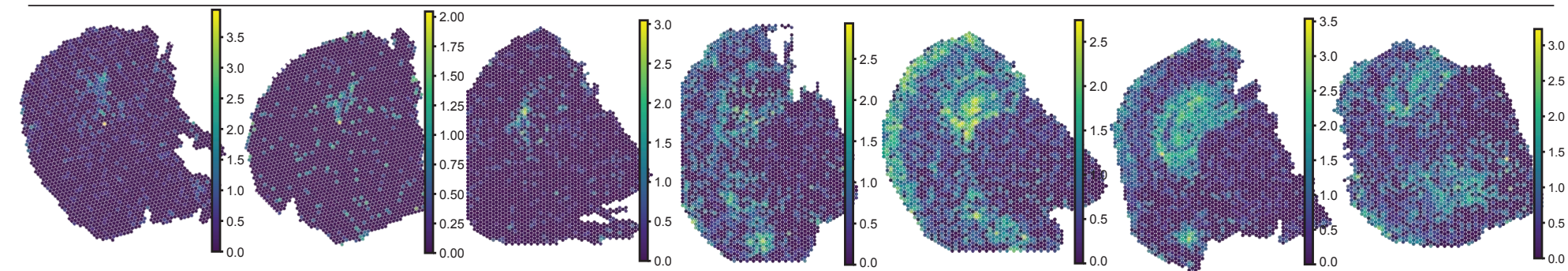

C

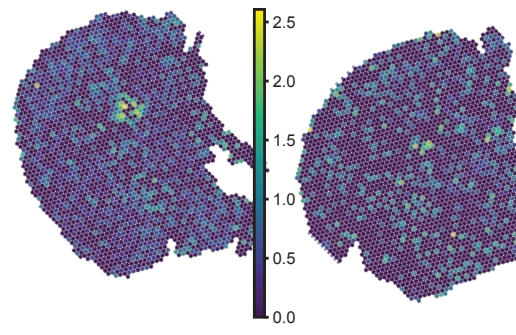

d

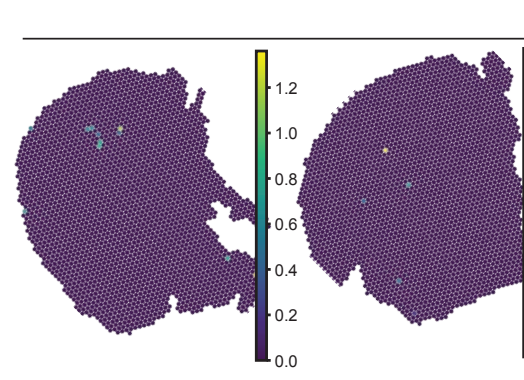

H2-D1

\section{CK-P25}
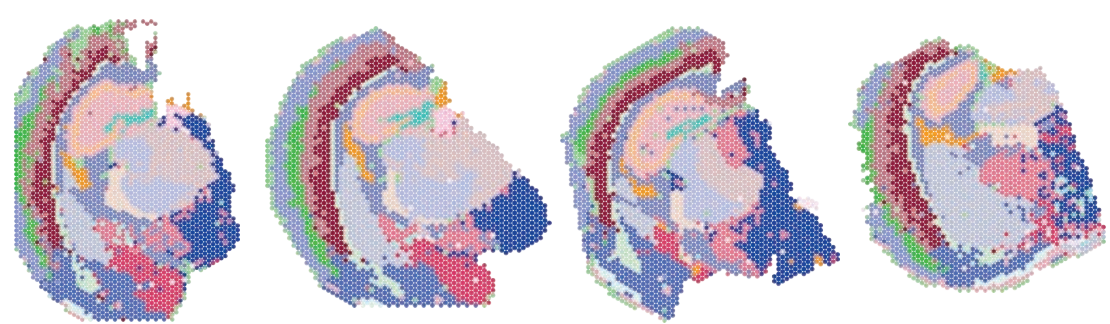

Ifitm3

12-D1
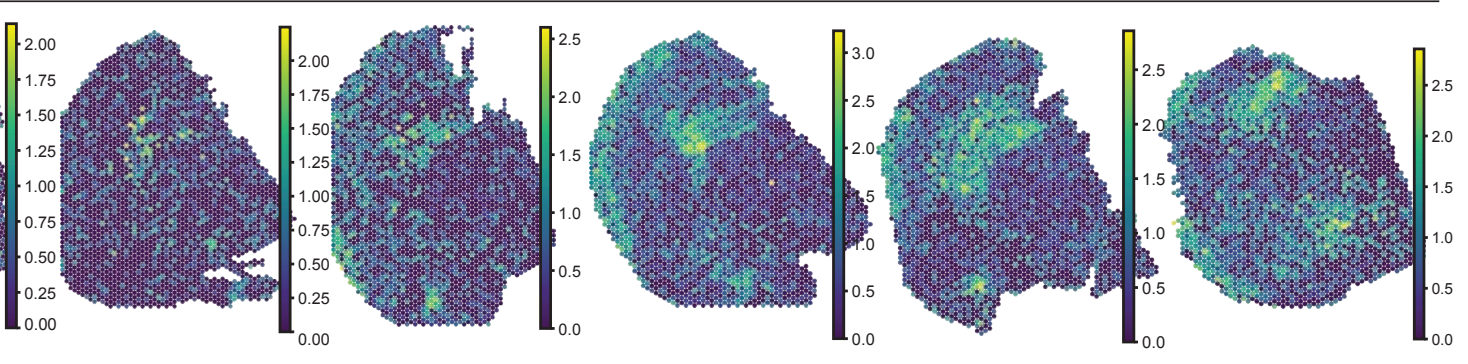

CxCl10

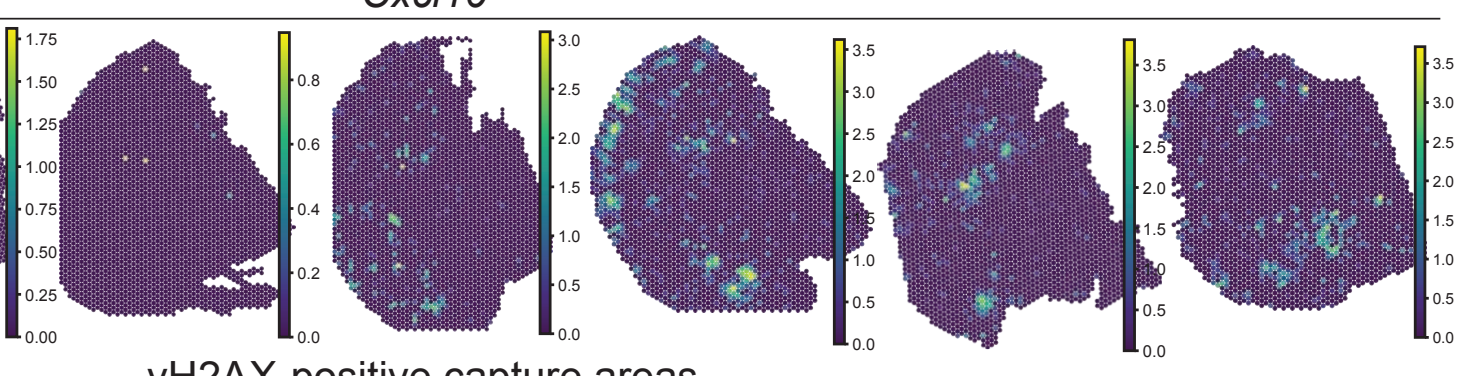

yH2AX-positive capture areas
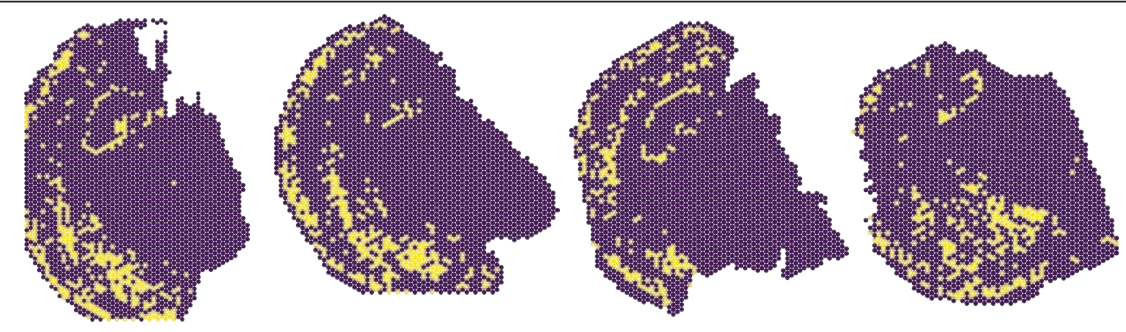

\section{Supplementary Figure 9.}

a. Distribution of spatial clusters for each sample.

b-d. Expression of reactive microglia genes and DSB-bearing neuron genes (b) Ifitm3, (c) H2-D1, and (c) Cxcl10 for each sample.

e. Distribution of $\mathrm{yH} 2 \mathrm{AX}$-positive capture areas for each CK-p25 sample. 

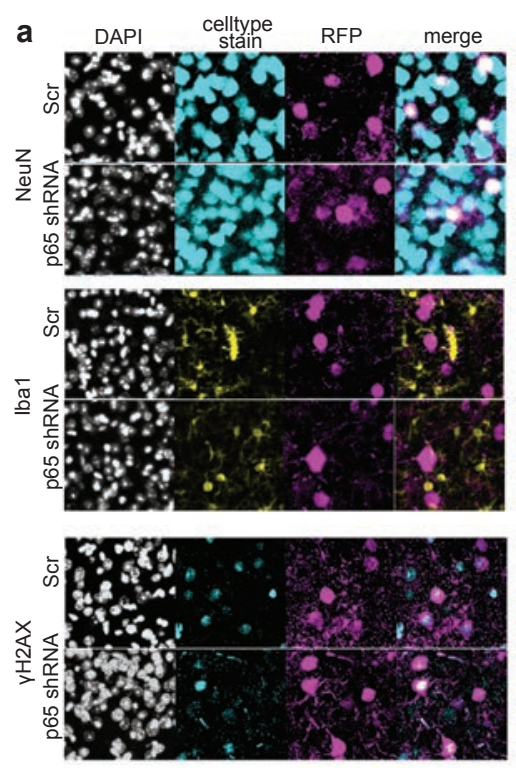

\section{Supplementary Figure 10.}
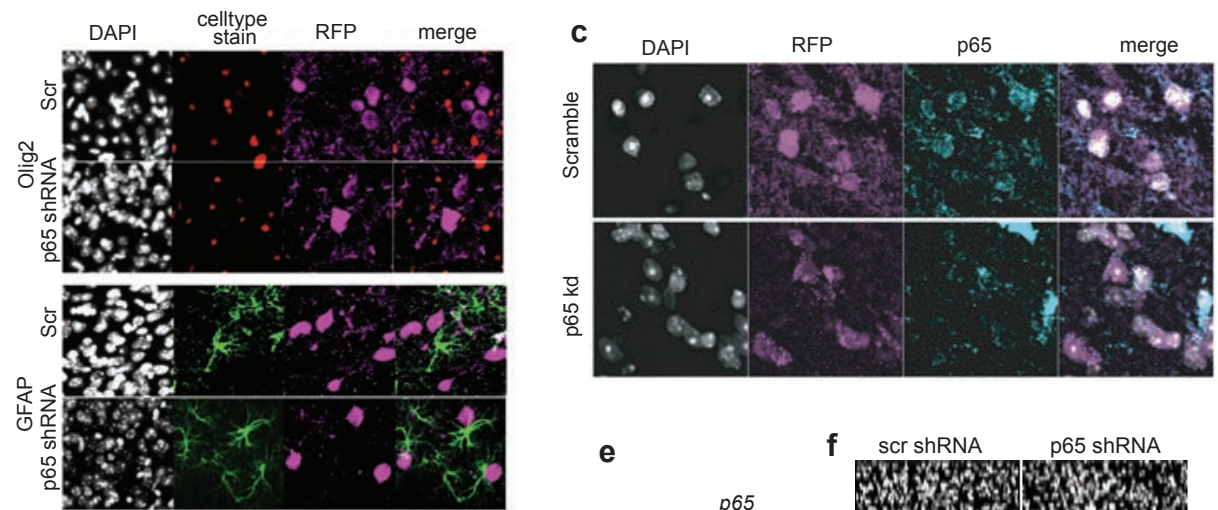

d
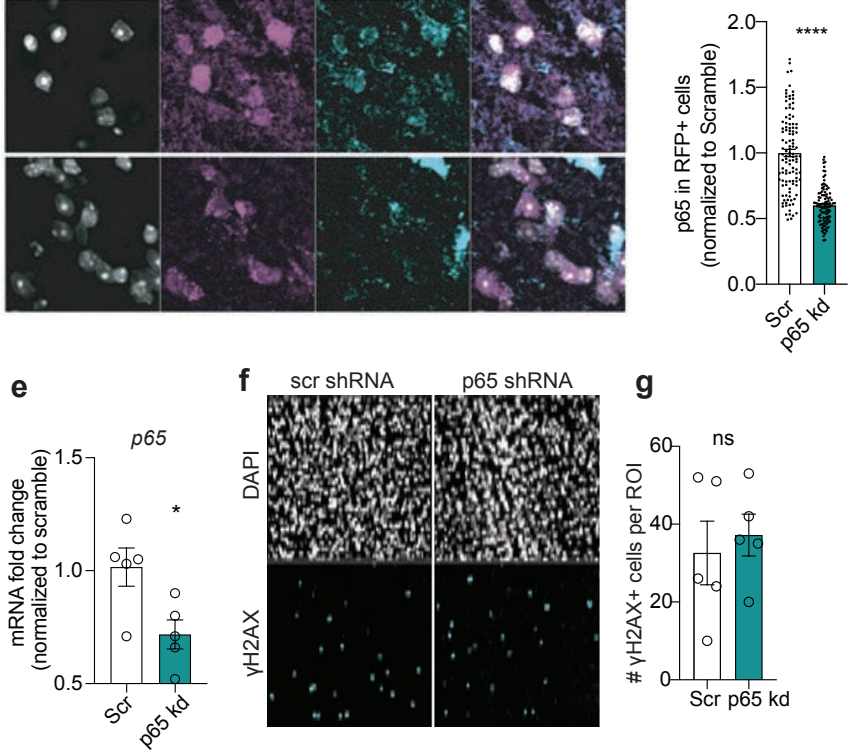

a. Representative images of cell type specific immunostaining and RFP immunostaining in scramble and p65kd cortex. Top to bottom, left to right:

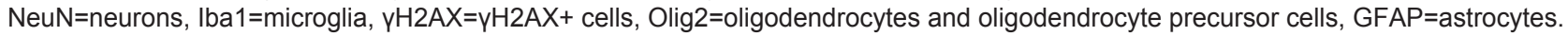

b. Quantification of cell type distribution of RFP+ cells. Total number of RFP+ cells are quantified per image, then the fraction co-stained for a celltype marker are calculated. Each datapoint represents one mouse.

c. P65 and RFP immunostaining in scramble and p65kd CK-p25 cortex.

d. Quantification of p65 mean intensity. Analysis was performed on three animals, two sections each. Scramble $n=113$ cells, p65kd $n=103$ cells.

e. qRT-PCR of p65 in RFP+ NeuN+ nuclei from scramble and shp65-treated CK-p25 mice.

f. Representative images of $\mathrm{yH} 2 \mathrm{AX}$ immunostaining in $\mathrm{p} 65 \mathrm{kd}$ and scramble $\mathrm{CK}-\mathrm{p} 25$ cortex.

g. Number of $\mathrm{yH} 2 \mathrm{AX}+$ per image are quantified. Each data point represents one mouse.

Error bars represent standard error of mean (S.E.M.); ${ }^{* \star *} \mathrm{P}<0.0001,{ }^{* \star} \mathrm{P}<0.001,{ }^{* \star} \mathrm{P}<0.01,{ }^{*} \mathrm{P}<0.05$, n.s. not significant. Student's t-test (d,e,g). 
a

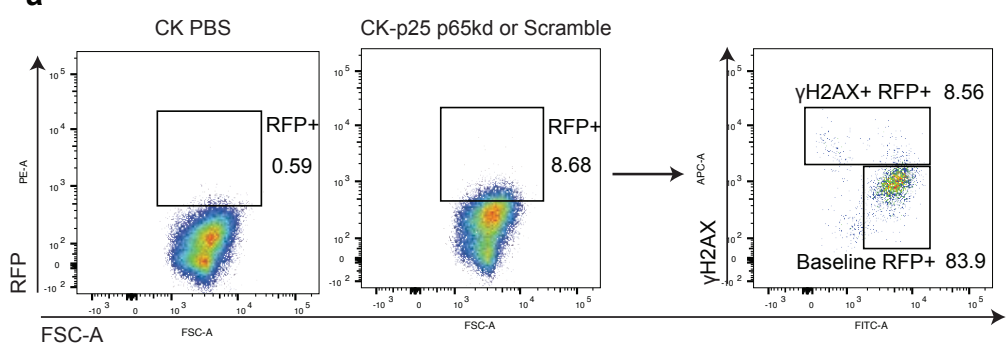

d

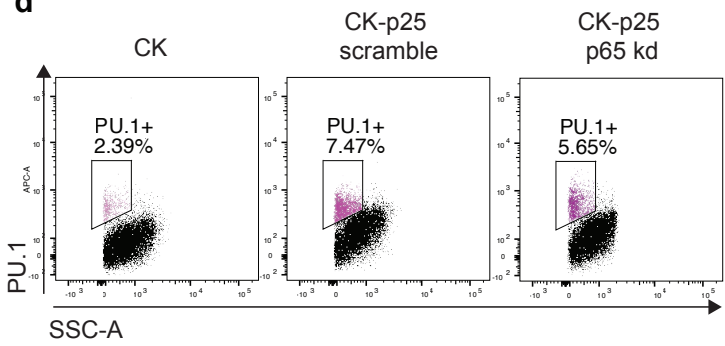

b
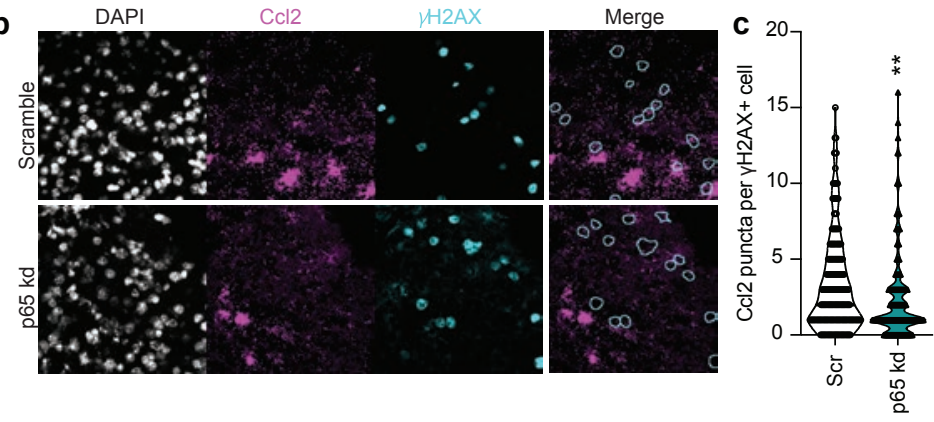

f

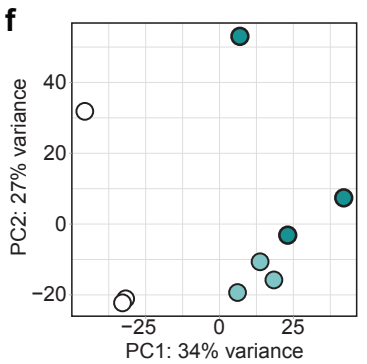

g
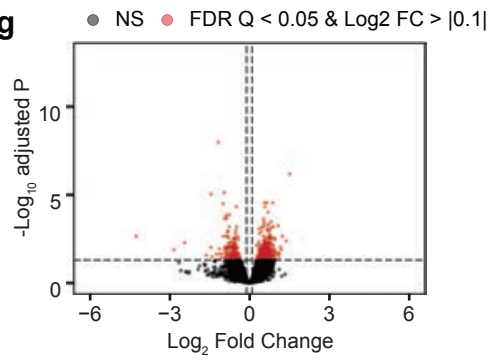

\section{Supplementary Figure 11.}

a. Sorting schematic for RFP+ $\mathrm{yH} 2 \mathrm{AX}+$ and $\mathrm{yH} 2 \mathrm{AX}$ - neurons. 30,000 nuclei were collected for each gate for each animal.

b. Representative images of Ccl2 RNAscope combined with $\mathrm{yH} 2 \mathrm{AX}$ immunofluorescence in Scramble and p65kd cortex.

c. Quantification of Ccl2 puncta per $\mathrm{yH} 2 \mathrm{AX}+$ cell. Each datapoint represents one cell ( $\mathrm{n}=197$ for scramble, $n=157$ for $p 65 \mathrm{kd}) .20-40$ cells were analyzed per mouse.

d. Sorting schematic for Pu.1+ nuclei for RNA-sequencing.

e. Quantification of total percent Pu.1+. One datapoint represents one mouse.

f. PCA plot of normalized gene expression matrix from Pu.1+ bulk RNA-sequencing.

g. Volcano plot from P65 vs. Scramble contrast.

Error bars represent standard error of mean (S.E.M.); ${ }^{* * *} \mathrm{P}<0.0001,{ }^{* * *} \mathrm{P}<0.001,{ }^{* *} \mathrm{P}<0.01,{ }^{*} \mathrm{P}<0.05$, ns not significant. One-way ANOVA followed by Tukey's test for multiple comparisons (e). 


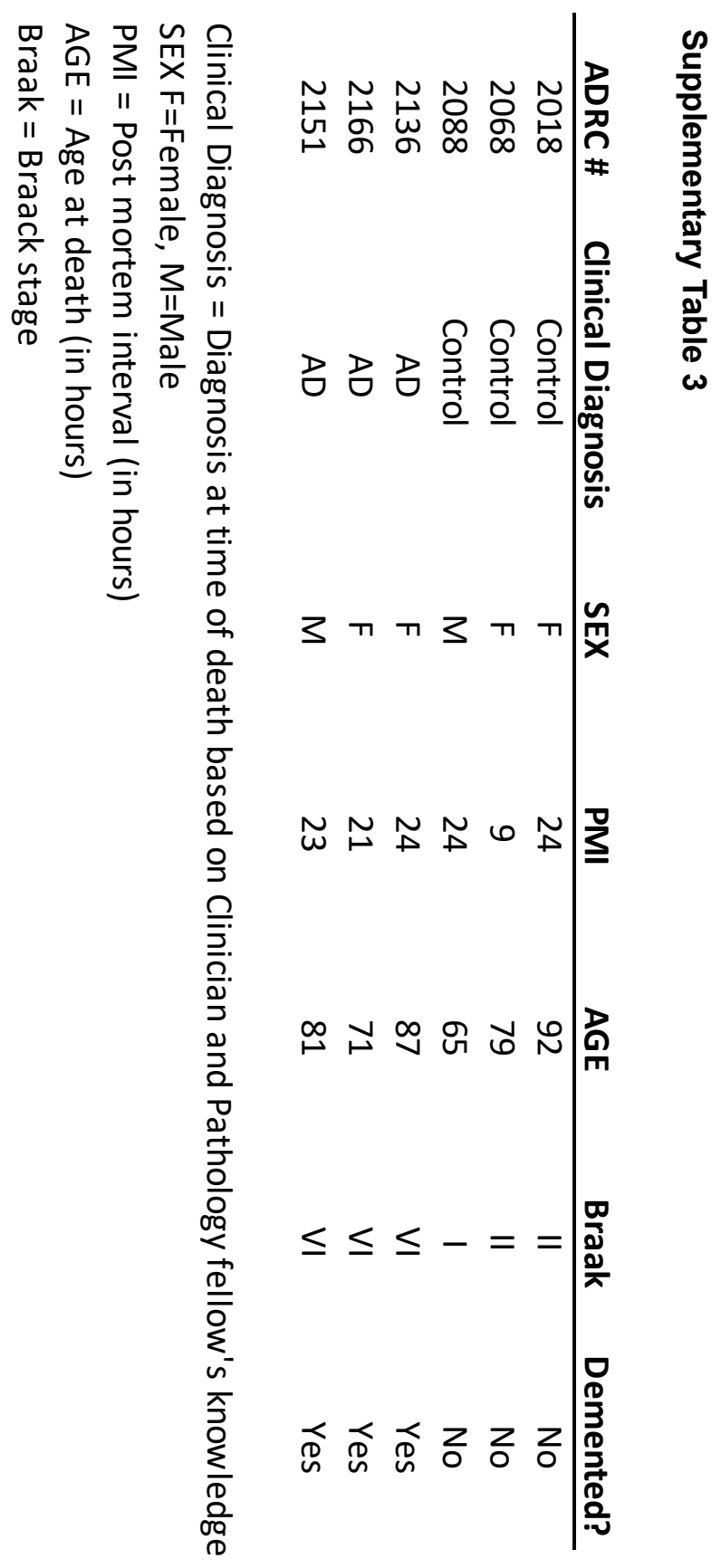



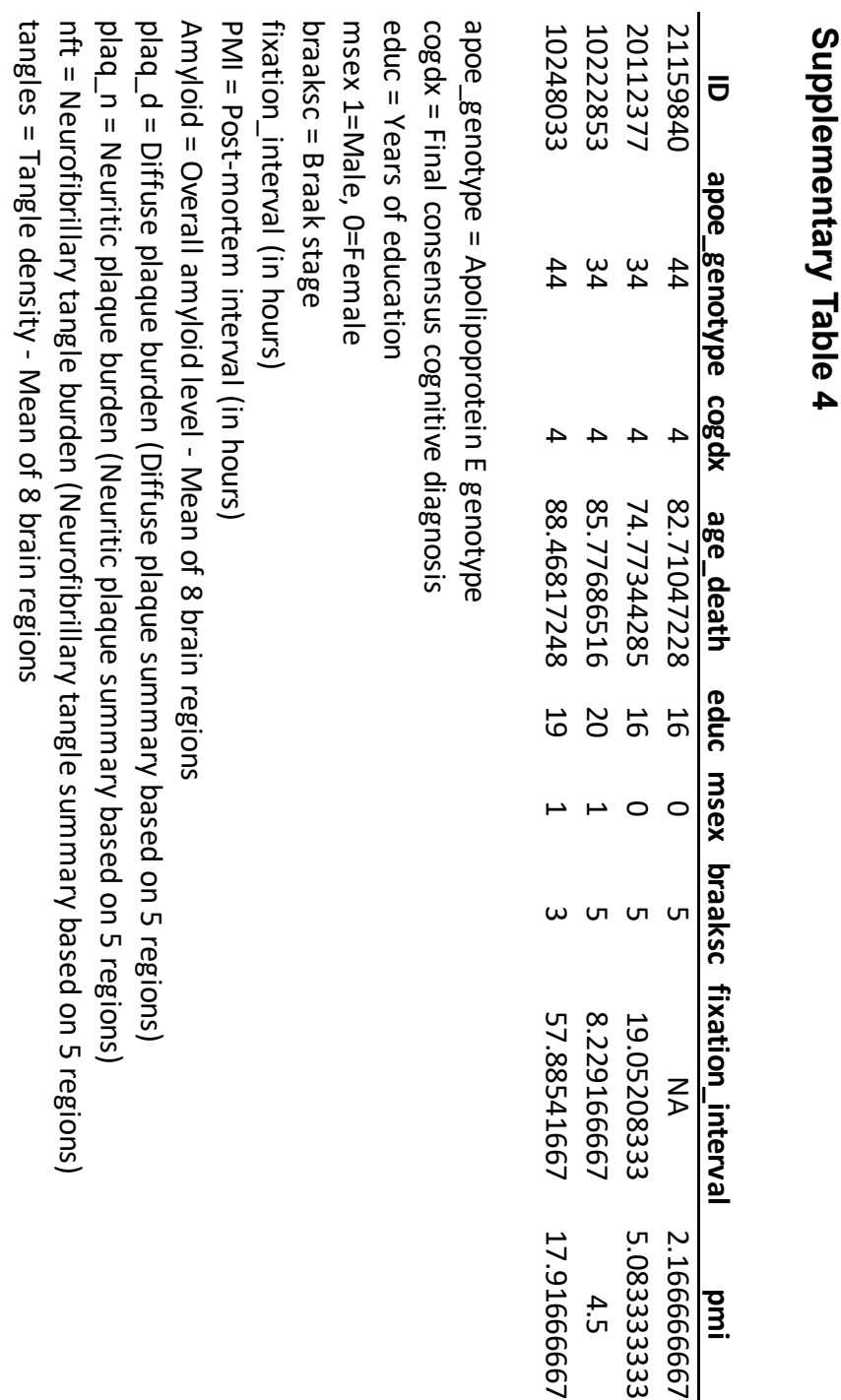